November 1996

PDMI - 3/1996

Berlin Sfb288 No.238

q-alg/9611010

\title{
Vertex Operators - From a Toy Model to Lattice Algebras
}

\author{
ANDREI G. Bytsko * \\ Institut für Theoretische Physik, Freie Universität Berlin \\ Arnimallee 14, 14195 Berlin, Germany \\ and \\ Steklov Mathematical Institute, Fontanka 27, \\ St.Petersburg 191011, Russia \\ VOLKER SCHOMERUS ** \\ II. Institut für Theoretische Physik, Universität Hamburg \\ Luruper Chaussee 149, 22761 Hamburg, Germany
}

\begin{abstract}
Within the framework of the discrete Wess-Zumino-Novikov-Witten theory we analyze the structure of vertex operators on a lattice. In particular, the lattice analogues of operator product expansions and braid relations are discussed. As the main physical application, a rigorous construction for the discrete counterpart $g_{n}$ of the group valued field $g(x)$ is provided. We study several automorphisms of the lattice algebras including discretizations of the evolution in the WZNW model. Our analysis is based on the theory of modular Hopf algebras and its formulation in terms of universal elements. Algebras of vertex operators and their structure constants are obtained for the deformed universal enveloping algebras $U_{q}(\mathfrak{G})$. Throughout the whole paper, the abelian WZNW model is used as a simple example to illustrate the steps of our construction.
\end{abstract}

* e-mail: bytsko@physik.fu-berlin.de, bytsko@pdmi.ras.ru

** e-mail: vschomer@x4u.desy.de 


\section{Contents}

\begin{tabular}{lll}
\hline INTRODUCTION & 2
\end{tabular}

2 HOPF ALGEBRAS AND VERTEX OPERATORS

2.1 Semi-simple modular Hopf algebras. . . . . . . . . . . . . . . . 5

2.2 Universal elements and $R$-matrix formalism. . . . . . . . . . . . . . . . . . 7

2.3 Vertex operators and their structure data. . . . . . . . . . . . . . . . . . . . . . . . . . . . . . . . .

2.4 Gauge transformations of vertex operators. . . . . . . . . . . . . . . . . 12

2.5 On the construction of vertex operators. . . . . . . . . . . . . . 13

$2.6 \quad$ Vertex operators for $\mathcal{Z}_{q} \ldots \ldots \ldots \ldots \ldots \ldots \ldots$

3 A TOY MODEL FOR THE DISCRETE WZNW THEORY 15

3.1 Properties of chiral vertex operators. . . . . . . . . . . . . . 15

3.2 Second chirality. . . . . . . . . . . . . . . . . . . . 16

3.3 *-operation for chiral vertex operators. . . . . . . . . . . . . . . . . . 18

3.4 Quantum group valued field $g . \ldots \ldots \ldots \ldots$. . . . . . . . . . . 19

3.5 Toy model for $\mathcal{Z}_{q} \ldots \ldots \ldots \ldots \ldots \ldots \ldots \ldots$

4 REVIEW ON LATTICE CURRENT ALGEBRAS 22

4.1 Definition of lattice current algebras. . . . . . . . . . . . . . . 23

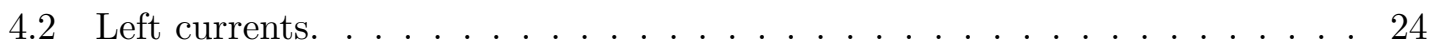

4.3 Holonomies and monodromies . . . . . . . . . . . . . . . . . . . . . . . . . . 24

$4.4 \quad$ Current algebra for $\mathcal{Z}_{q} \ldots \ldots \ldots \ldots \ldots \ldots \ldots$

5 VERTEX OPERATORS ON A LATTICE 26

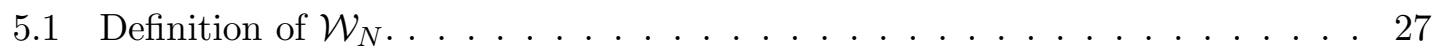

5.2 Vertex operators at different sites. . . . . . . . . . . . . . . . 28

5.3 Extension on a covering of the circle. . . . . . . . . . . . . . . . 30

5.4 Construction of the local field $g_{n} \ldots \ldots \ldots \ldots \ldots \ldots \ldots$

$5.5 \quad$ Lattice vertex operators for $\mathcal{Z}_{q} \ldots \ldots \ldots \ldots \ldots \ldots$

6 AUTOMORPHISMS AND DISCRETE DYNAMICS

6.1 Remarks on the classical continuum limit. . . . . . . . . . . . . . 34

6.2 Automorphisms induced by the ribbon element . . . . . . . . . . . . . . 35

6.3 Discrete dynamics. . . . . . . . . . . . . . . . . . . . . 36

$6.4 \quad U(1)$-WZNW model. $\ldots \ldots \ldots \ldots \ldots$

$\begin{array}{ll}\text { CONCLUSION } & 41\end{array}$

APPENDIX: SOME PROOFS AND FURTHER RELATIONS 42

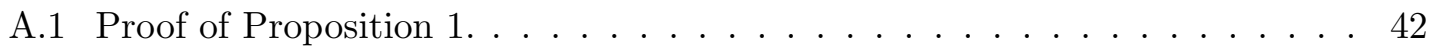

A.2 Proof of Proposition 3. . . . . . . . . . . . . . . . . . . . . . . . . . 42

A.3 $\quad$ Structure data for left chiral vertex operators. . . . . . . . . . . . . . . . . . 44

A.4 Properties of the field $g . \ldots \ldots \ldots \ldots \ldots \ldots$. . . . . . . . . . . . . . . . . . . . . 45

A.5 Properties of lattice vertex operators. . . . . . . . . . . . . . . . . 46

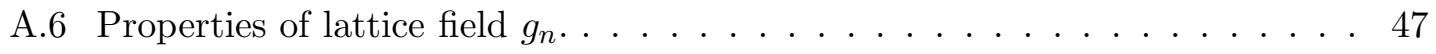




\section{INTRODUCTION}

Quantization of the WZNW model. The Wess-Zumino-Novikov-Witten (WZNW) model [50, 46, 51, 41 is one of the most famous examples of a rational conformal field theory (CFT) [14, 42, 44]. On the classical level it describes some time evolution for a field $g(x)$ mapping points $x$ of the circle $\mathbf{S}^{1}$ into a compact Lie group $G$. Among the dynamical variables of the theory, the currents $j^{r}(x)=g^{-1} \partial_{-} g, j^{l}=\left(\partial_{+} g\right) g^{-1}$ are of particular interest. In contrast to the field $g$, the currents $j^{r}$ and $j^{l}$ are chiral, so that $\partial_{+} j^{r}=0$ and $\partial_{-} j^{l}=0$. Moreover, their Poisson structure is well known to give rise to two commuting copies of Kac-Moody (KM) algebras. Even though numerous papers have been devoted to the quantization of the WZNW-model (e.g. [16, 26, 10, 36, 13, 20, 21, 35]), a rigorous construction of the continuum theory (which requires field strength renormalization) is not fully understood. This motivates the search for lattice regularizations of the theory (i.e., the circle $\mathbf{S}^{1}$ is replaced by a periodic lattice with lattice spacing $a$ ) which preserve much of the symmetry structure of the continuum WZNW-model. One may construct appropriate discretizations of the classical model (i.e., $\hbar=0$ ) first and then quantize the classical lattice theory to obtain a well defined discrete quantum theory (i.e., $\hbar \neq 0, a \neq 0$ ). Investigation of the latter is expected to provide insights into the structure of the continuum model. A final step would involve performing the limit $a \rightarrow 0$ while keeping $\hbar \neq 0$.

The realization of this program was started in [4, 5, 27] where a lattice regularization of the Kac-Moody algebra has been proposed. Classical and quantum lattice current algebras were further investigated in [33, 6]. Our aim here is to extend the analysis of [6] by introducing chiral vertex operators. In comparison with the current algebra, the algebras of vertex operators contain (a finite number of) additional generators. Within these larger algebras we will be able to prepare a discrete analogue of the group valued field $g(x)$ by combining left- and right chiral vertex operators.

Quantum symmetry structure of the WZNW model. Let us recall that solutions of the classical Yang-Baxter equation appear already in the Poisson structure of the classical lattice current algebras (see [33] and references therein). After quantization, quantum groups and quantum universal enveloping algebras $\mathcal{G}$ 円 are expected to emerge. Throughout this paper we will meet (global and local) objects (the monodromies $M^{\alpha}$ and a discrete field $N_{n}$, see below) whose nature reflects a quantum algebraic structure as well as an object (the discrete field $g_{n}$ ) which displays features of a quantum group. The corresponding deformation parameter is of the form $q=\exp \{i \gamma \hbar\}$ with $\gamma=\pi /(k+\nu)$ (where $k$ is the level of the KM algebra and $\nu$ is the dual Coxeter number of $G$ ) and does not depend on the lattice spacing $a$. Therefore, the quantum group structures of the continuum and lattice WZNW model coincide. It is also worth mentioning that some aspects of the quantum symmetry structure survive reductions to other theories so that part of what we describe below may be compared with studies of the quantum Liouville and Toda models [37, 29, 11, 23].

Remarks on lattice current algebras. Before we summarize our results, let us briefly review the discretization used in [6] for the chiral currents $j^{l}(x), j^{r}(x)$. Recall that the latter are Lie-algebra valued fields which depend periodically on the variable $x$. Instead of working with these standard variables, we prefer to pass to the fields $j^{r}(x)$ and $\eta(x)=j^{r}(x)-j^{l}(x)$ and describe their lattice counterparts. Our lattice divides the circle into $N$ links of length $a=2 \pi / N$. So there are $N$ vertices at the points $x=a n$ which are numbered by

\footnotetext{
${ }^{1}$ For shortness, we will often refer to $\mathcal{G}$ as quantum algebra.
} 
$n=0, \ldots, N-1, N \equiv 0$ and the $n^{\text {th }}$ link runs from the $(n-1)^{\text {st }}$ vertex to the $n^{\text {th }}$ We may discretize the field $\eta(x)$ by the simple prescription $\eta_{n}:=\int_{\left(n-\frac{1}{2}\right) a}^{\left(n+\frac{1}{2}\right) a} \eta(x) d x=a \eta(n a)+O\left(a^{2}\right)$ so that the lattice field $\eta_{n}$ has values in a tensor product of $N$ copies of the Lie algebra which are assigned to the $N$ vertices on the lattice. For the right chiral current $j^{r}(x)$ the discretization scheme is different. In this case we encode the information about the field in the holonomies along links, i.e., we define the lattice field $j_{n}^{r}$ by

$$
j_{n}^{r}:=\operatorname{Pexp}\left(\int_{n} j^{r}(x) d x\right) .
$$

Here $\int_{n}$ denotes integration along the $n^{\text {th }}$ link. By construction, this classical lattice field $j_{n}^{r}$ has values in the Lie group. The rather different treatment of the fields $\eta(x)$ and $j^{r}(x)$ may be understood from the Poisson structure of the classical theory, which is ultralocal for $\eta(x)$ but contains terms proportional to $\delta^{\prime}(x-y)$ if the field $j^{r}(x)$ is involved (see 四, 33, 6]).

When we pass to the quantum theory, the functions on the space of field configurations become operators and generate some non-commutative algebra $\mathcal{K}_{N}$. More concretely, the algebra $\mathcal{K}_{N}$ is generated from the quantum lattice fields $J_{n}^{r}, N_{n}$ which correspond to the classical fields $j_{n}^{r}, \eta_{n}$ described above. We review the explicit definition of lattice current algebras in Section 4. Let us only mention here that a very elegant formulation for commutation relations of the quantum operators can be given in the $R$-matrix language.

In mathematical terms, one has to regard the quantum fields $N_{n}$ and $J_{n}=J_{n}^{r}$ as objects in the tensor product $\mathcal{G}_{a} \otimes \mathcal{K}_{N}$ of the deformed universal enveloping algebra $\mathcal{G}_{a}=U_{q}(\mathfrak{G})$ with the lattice current algebra $\mathcal{K}_{N}$. We can understand this by looking at the classical lattice field $j_{n}^{r}$, for instance. It was constructed as the holonomy of the Lie-algebra valued field $j^{r}(x)$ and may be evaluated with irreducible representations of the Lie algebra. Let us denote such representations by $\tau^{I}$ and introduce the symbols $V^{I}, \delta_{I}$ for their carrier spaces and dimensions, respectively. Then we see that $j_{n}=j_{n}^{r}$ gives rise to $\delta_{I} \times \delta_{I^{-}}$-matrices $j_{n}^{I}$ of dynamical variables. Accordingly, the corresponding quantum operators $J_{n}^{I}$ are matrices of generators for $\mathcal{K}_{N}$ which is to say that $J_{n}^{I} \in \operatorname{End}\left(V^{I}\right) \otimes \mathcal{K}_{N}$. All these objects $J_{n}^{I}$ may be assembled back into one universal element $J_{n} \in \mathcal{G}_{a} \otimes \mathcal{K}_{N}$. More details will be presented later; we anticipated this heuristic discussion of universal elements only to prepare for some formulae below.

One of the main aims in [6] was to develop a complete representation theory for lattice current algebra $\mathcal{K}_{N}$. It turned out that $\mathcal{K}_{N}$ possesses a family of irreducible *representations on vector spaces $W_{N}^{I J}$ with labels $I, J$ running through classes of finitedimensional, irreducible representations of $U_{q}(\mathfrak{G})$. Two such labels are needed because of the two chiralities in the current algebra. Furthermore, the algebra $\mathcal{K}_{N}$ was found to admit two families of local co-actions $\Lambda_{n}^{r}, \Lambda_{n}^{l}: \mathcal{K}_{N} \mapsto \mathcal{G}_{a} \otimes \mathcal{K}_{N}$ of the Hopf algebra $\mathcal{G}_{a}$. They may be considered as a special case of the more general lattice fusion products in [6] and give rise to a notion of tensor products for representations of $\mathcal{K}_{N}$ (see also [45] for related results).

Vertex operators on a lattice. Product structures in the representation theory are precisely what is needed to initiate a theory of vertex operators. More technically, we employ the homomorphisms $\Lambda_{n}^{r}, \Lambda_{n}^{l}$ in extending the lattice current algebra $\mathcal{K}_{N}$ by chiral vertex operators $\Phi_{n}^{r}, \Phi_{n}^{l}$ so that the following intertwining relations hold for both chiralities $\alpha=$ $r, l$,

$$
A \Phi_{n}^{\alpha}=\Phi_{n}^{\alpha} \Lambda_{n}^{\alpha}(A) \quad \text { for all } \quad A \in \mathcal{K}_{N}
$$


The elements $\Phi_{n}^{\alpha}$ generate an extension $\mathcal{W}_{N}$ of the lattice current algebra $\mathcal{K}_{N} \subset \mathcal{W}_{N}$. Since $\Lambda_{n}^{\alpha}(A)$ is an element of $\mathcal{G}_{a} \otimes \mathcal{K}_{N}$ and hence also of the extension $\mathcal{G}_{a} \otimes \mathcal{W}_{N}$, the product on the r.h.s. of (1.1) is well defined for $\Phi_{n}^{\alpha} \in \mathcal{G}_{a} \otimes \mathcal{W}_{N}$. On the l.h.s., $A=e \otimes A \in \mathcal{G}_{a} \otimes \mathcal{K}_{N}$ with $e \in \mathcal{G}_{a}$ being the unit element.

Our vertex operators $\Phi_{n}^{\alpha}$ on the lattice possess a number of properties which are all closely related to properties of vertex operators in the continuum theory. Let us highlight some of them without going into a detailed discussion.2

1. Lattice vertex operators $\Phi_{n}^{\alpha}$ at a fixed lattice site obey operator product expansions of the form

$$
\stackrel{2}{\Phi} \stackrel{1}{\Phi}_{n}^{r}=F_{r} \Delta_{a}\left(\Phi_{n}^{r}\right) \quad \text { and } \quad \stackrel{1}{\Phi}{ }_{n}^{l} \stackrel{2}{\Phi}=F_{n} \Delta_{a}\left(\Phi_{n}^{l}\right)
$$

As usual, the notation $\stackrel{1}{\Phi_{n}^{\alpha}}$ means that we regard the vertex operator $\Phi_{n}^{\alpha}$ as an element of $\mathcal{G}_{a} \otimes \mathcal{G}_{a} \otimes \mathcal{W}_{N}$ with trivial entry in the second tensor factor etc. We have also used the shorthand $\Delta_{a}\left(\Phi_{n}^{\alpha}\right)=(\Delta \otimes i d)\left(\Phi_{n}^{\alpha}\right) \in \mathcal{G}_{a} \otimes \mathcal{G}_{a} \otimes \mathcal{W}_{N}$ for the action of the co-product on the first tensor factor of $\Phi_{n}^{\alpha}$. The objects $F_{\alpha}$ are analogues of the fusion matrix in the continuum theory. We describe their general properties and, in particular, their relation with $6 j$-symbols in Section 2.

2. Lattice vertex operators $\Phi_{n}^{\alpha}$ assigned to different lattice sites obey braid relations

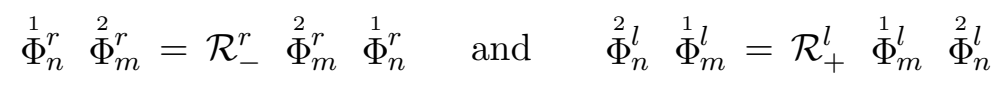

for all $0 \leq n<m<N$. Here the objects $\mathcal{R}_{ \pm}$play the role of the braiding matrix in the continuum theory. Let us add that lattice vertex operators of different chirality commute for all $n, m$. Furthermore, $\Phi_{n}^{\alpha}$ commute with $N_{m}$ for $m \neq n$ and with $J_{m}$ for $m \neq n, n+1$, that is, the vertex operators have local exchange relations with elements of the current algebra.

3. Lattice vertex operators $\Phi_{n}^{\alpha}, \alpha=r, l$, satisfy the following difference equation

$$
\Phi_{n+1}^{\alpha}=\Phi_{n}^{\alpha} J_{n+1}^{\alpha}
$$

In the naive continuum limit, we have $J_{n}^{\alpha}=e \otimes e-a J^{\alpha}(x)+O\left(a^{2}\right)$ with $x=$ an and the difference equation becomes a differential equation which expresses $\partial_{x} \Phi^{\alpha}(x)$ as a (normal ordered) product of $\Phi^{\alpha}(x)$ and $J^{\alpha}(x)$. Such an equation is well known for the quantized continuum theory.

As one may infer from the third property in this short list, lattice vertex operators (much like their continuum counterparts) cannot be periodic. Indeed, starting from $\Phi_{0}^{\alpha}$ an iterated application of eq. (1.4) gives

$$
\Phi_{N}^{\alpha} \equiv \Phi_{0}^{\alpha} M^{\alpha} \quad \text { with } \quad M^{\alpha}=J_{1}^{\alpha} \ldots J_{N}^{\alpha}
$$

The objects $M^{\alpha}, \alpha=r, l$, are called chiral monodromies. Actually, the lattice rotation $n \mapsto n+N$ gives rise to an inner automorphism of the algebra of vertex operators which acts trivially on the lattice fields $J_{n}^{\alpha}$ and $N_{n}$. We show in Section 6 that this automorphism

\footnotetext{
${ }^{2}$ A construction of (non-chiral) vertex operators for infinite open lattices has been suggested in [45]. Some properties of these vertex operators are similar to what we shall consider here. However, these are different structures, in particular, because for a finite lattice the current algebra $\mathcal{K}_{N}$ has a non-trivial center $\mathcal{C}$. An action of our vertex operators on elements from $\mathcal{C}$ will play a crucial role in the theory.
} 
can be generated by conjugation with a unitary element $v$. The latter is constant on the irreducible representation spaces $W_{N}^{I J}$ of the lattice current algebra $\mathcal{K}_{N}$ and its value

$$
\mathrm{v}^{I J}=e^{2 \pi i\left(h_{J}-h_{I}\right)}
$$

can be expressed in terms of the conformal dimensions $h_{I}$ of the WZNW model. This leads us to identify $v$ with the operator $\exp \left\{2 \pi\left(L_{0}-\bar{L}_{0}\right)\right\}$ which generates rotations by $2 \pi$ in the continuum theory. In the lattice theory $v$ is obtained from quantum traces of chiral monodromies $M^{\alpha}$ and is related to the ribbon element of $U_{q}(\mathfrak{G})$.

It will be shown in Sections 3 and 5 that the field $\mathcal{S}_{a}\left(\Phi_{n}^{l}\right) \Phi_{n}^{r}$. diagonal subspace $\bigoplus_{K} W_{N}^{\bar{K} K}$. Let us denote this restriction by $g_{n}$ which suggests that it is a quantum lattice analogue of the group valued field $g(x)$ in the WZNW model. In fact, our analysis will reveal that $g_{n}$ is a local and quantum group valued field, i.e.

$$
\stackrel{1}{g}_{n} \stackrel{2}{g}_{m}=\stackrel{2}{g}_{m} \stackrel{1}{g}_{n} \quad(n \neq m) \quad \text { and } \quad R \quad \stackrel{2}{g}_{n} \stackrel{1}{g}_{n}=\stackrel{1}{g}_{n} \stackrel{2}{g}_{n} R
$$

Moreover, $g_{n}$ turns out to be periodic. In contrast to the chiral currents $j^{\alpha}(x)$, the time evolution of the group valued field $g(x)$ is described by a nontrivial second order differential equation. Its discrete analogue is discussed in Section 6.

Before we address the full lattice theory we explain some basic constructions in a simple toy model (cf. Section 3). Here one studies the algebra generated by the monodromies $M^{r}, M^{l}$ instead of the whole (lattice) current algebra and universal (deformed) tensor operators for the quantum algebra $\mathcal{G}$ as simple examples of vertex operators [2, 22, 3, 19]. This finite dimensional toy model may be regarded as a special case of the discrete WZNW theory where $N=1$ and it describes the non-local degrees of freedom for an arbitrary number $N$ of lattice sites. Objects and relations of the toy model admit for a nice pictorial presentation which, in particular, brings new light into the shadow world [39].

\section{HOPF ALGEBRAS AND VERTEX OPERATORS}

2.1 Semi-simple modular Hopf algebras. By definition, a Hopf algebra is a quadruple $(\mathcal{G}, \epsilon, \Delta, \mathcal{S})$ of an associative algebra $\mathcal{G}$ (the "symmetry algebra") with unit $e \in \mathcal{G}$, a one-dimensional representation $\epsilon: \mathcal{G} \mapsto \mathbb{C}$ (the "co-unit"), a homomorphism $\Delta: \mathcal{G} \mapsto \mathcal{G} \otimes \mathcal{G}$ (the "co-product") and an anti-automorphism $\mathcal{S}: \mathcal{G} \mapsto \mathcal{G}$ (the "antipode"). These objects obey a set of basic axioms which can be found, e.g., in [1, 49]. The Hopf algebra $(\mathcal{G}, \epsilon, \Delta, \mathcal{S})$ is called quasi-triangular if there is an invertible element $R \in \mathcal{G} \otimes \mathcal{G}$ such that

$$
\begin{array}{r}
R \Delta(\xi)=\Delta^{\prime}(\xi) R \text { for all } \xi \in \mathcal{G}, \\
(i d \otimes \Delta)(R)=R_{13} R_{12} \quad, \quad(\Delta \otimes i d)(R)=R_{13} R_{23} .
\end{array}
$$

Here $\Delta^{\prime}(\xi)=P \Delta(\xi) P$, with $P$ being the permutation, i.e., $P(\xi \otimes \eta) P=\eta \otimes \xi$ for all $\xi, \eta \in \mathcal{G}$, and we are using the standard notation for the elements $R_{i j} \in \mathcal{G} \otimes \mathcal{G} \otimes \mathcal{G}$.

For a ribbon Hopf-algebra one postulates, in addition, the existence of a certain invertible central element $v \in \mathcal{G}$ (the "ribbon element") which factorizes $R^{\prime} R \in \mathcal{G} \otimes \mathcal{G}$ ( here $\left.R^{\prime}=P R P\right)$, in the sense that

$$
R^{\prime} R=(v \otimes v) \Delta\left(v^{-1}\right), \quad \mathcal{S}(v)=v, \quad \epsilon(v)=1
$$

\footnotetext{
${ }^{3}$ We use the notation $\mathcal{S}_{a}\left(\Phi_{n}^{l}\right)=(\mathcal{S} \otimes i d)\left(\Phi_{n}^{l}\right)$ with $\mathcal{S}$ being the antipode of $\mathcal{G}$.
} 
(see [48, 40] for details). We want this structure to be consistent with a $*$-operation on $\mathcal{G}$. To be more precise, we require that

$$
R^{*}=\left(R^{-1}\right)^{\prime}=P R^{-1} P, \quad \Delta(\xi)^{*}=\Delta^{\prime}\left(\xi^{*}\right), \quad v^{*}=v^{-1} .
$$

This structure is of particular interest, since it appears in the theory of the quantized universal enveloping algebras $U_{q}(\mathfrak{G})$ when the complex parameter $q$ has values on the unit circle 43].

At this point we assume that $\mathcal{G}$ is semi-simple, so that every finite dimensional representation of $\mathcal{G}$ can be decomposed into a direct sum of finite dimensional, irreducible representations. From each equivalence class $[I]$ of irreducible representations of $\mathcal{G}$, we may pick a representative $\tau^{I}$, i.e., an irreducible representation of $\mathcal{G}$ on a $\delta_{I^{-}}$-dimensional Hilbert space $V^{I}$. The quantum $\operatorname{trace} \operatorname{tr}_{q}^{I}$ is a linear functional acting on elements $X \in \operatorname{End}\left(V^{I}\right)$ by

$$
\operatorname{tr}_{q}^{I}(X)=\operatorname{tr}^{I}\left(X \tau^{I}(w)\right)
$$

Here $\operatorname{tr}^{I}$ denotes the standard trace on $\operatorname{End}\left(V^{I}\right)$ with $\operatorname{tr}^{I}\left(e^{I}\right)=\delta_{I}$ and $w \in \mathcal{G}$ is a distinguished group-like element constructed from the ribbon element $v$ and the element $R$ by the formula $w^{-1}=v^{-1} \sum \mathcal{S}\left(r_{\varsigma}^{2}\right) r_{\varsigma}^{1}$, where the elements $r_{\varsigma}^{i}$ come from the expansion $R=\sum r_{\varsigma}^{1} \otimes r_{\varsigma}^{2}$.

Evaluation of the unit element $e^{I} \in \operatorname{End}\left(V^{I}\right)$ with $t r_{q}^{I}$ gives the quantum dimension, $d_{I}:=\operatorname{tr}_{q}^{I}\left(e^{I}\right)$, of the representation $\tau^{I}$. Furthermore, we assign a number $\mathrm{S}_{I J}$ to every pair of representations $\tau^{I}, \tau^{J}$ :

$$
\mathrm{S}_{I J}:=\vartheta\left(t r_{q}^{I} \otimes t r_{q}^{J}\right)\left(R^{\prime} R\right)^{I J} \quad \text { with } \quad\left(R^{\prime} R\right)^{I J}=\left(\tau^{I} \otimes \tau^{J}\right)\left(R^{\prime} R\right),
$$

with a suitable, real normalization factor $\vartheta$. The numbers $S_{I J}$ form the so-called $S$-matrix S. Modular Hopf algebras are ribbon Hopf algebras with an invertible $S$-matrix.f

Let us finally recall that the tensor product, $\tau \otimes \tau^{\prime}$, of two representations $\tau, \tau^{\prime}$ of a Hopf algebra is defined by

$$
\left(\tau \otimes \tau^{\prime}\right)(\xi)=\left(\tau \otimes \tau^{\prime}\right) \Delta(\xi) \quad \text { for all } \quad \xi \in \mathcal{G} .
$$

In particular, one may construct the tensor product $\tau^{I} \otimes \tau^{J}$ of two irreducible representations. According to our assumption that $\mathcal{G}$ be semi-simple, such tensor products of representations can be decomposed into a direct sum of irreducible representations.

Among all our assumptions on the structure of the Hopf-algebra $(\mathcal{G}, \epsilon, \Delta, \mathcal{S})$ (quasitriangularity, existence of a ribbon element $v$, semi-simplicity of $\mathcal{G}$ and invertibility of $S$-matrix S), semi-simplicity of $\mathcal{G}$ is the most problematic one. In fact it is violated by the algebras $U_{q}(\mathfrak{G})$ when $q$ is a root of unity. It is sketched in [7] how "truncation" can cure this problem, once the theory has been extended to weak quasi-Hopf algebras [43].

EXAmPLE: (Modular Hopf-algebra $\mathcal{Z}_{q}$ [6]) We wish to give one fairly trivial example for the algebraic structure discussed so far which comes from the group $\mathbb{Z}_{p}$. To be more precise, we consider the associative algebra $\mathcal{Z}_{q}$ generated by one element $h$ subject to the relation $h^{p}=e$. Co-product, co-unit and antipode for this algebra can be defined by

$$
\Delta(h)=h \otimes h, \mathcal{S}(h)=h^{-1}, \epsilon(h)=1 .
$$

\footnotetext{
${ }^{4}$ We fix $*$ on $\mathcal{G} \otimes \mathcal{G}$ by $(\xi \otimes \eta)^{*}=\xi^{*} \otimes \eta^{*}$. Following [43], we could define an alternative involution $\dagger$ on $\mathcal{G} \otimes \mathcal{G}$ which involves a permutation of components, i.e., $(\xi \otimes \eta)^{\dagger}=\eta^{\dagger} \otimes \xi^{\dagger}$ and $\xi^{\dagger}=\xi^{*}$ for all $\xi, \eta \in \mathcal{G}$. With respect to $\dagger, \Delta$ becomes an ordinary $*$-homomorphism and $R$ is unitary.

${ }^{5}$ If a diagonal matrix $\mathrm{T}$ is introduced according to $\mathrm{T}_{I J}=\varpi \delta_{I, J} d_{I}^{2} \tau^{I}(v)$ (with an appropriate complex factor $\varpi)$, then $S$ and $\mathrm{T}$ furnish a projective representation of the modular group $S L(2, \mathbb{Z})$.
} 
We observe that $\mathcal{Z}_{q}$ is a commutative semi-simple algebra. It has $p$ one-dimensional representations $\tau^{t}(h)=q^{t}, t=0, \ldots, p-1$, where $q$ is a root of unity, $q=e^{2 \pi i / p}$. We may construct characteristic projectors $P^{t} \in \mathcal{Z}_{q}$ for these representations according to

$$
P^{t}=\frac{1}{p} \sum_{m=0}^{p-1} q^{-t m} h^{m} \quad \text { for } \quad t=0, \ldots, p-1 .
$$

One can easily check that $\tau^{t}\left(P^{s}\right)=\delta_{t, s}$. The elements $P^{t}$ are employed to obtain a nontrivial $R$-matrix:

$$
R=\sum_{t, s=0}^{p-1} q^{t s} P^{t} \otimes P^{s} .
$$

It is easy to see that $\left(\tau^{t} \otimes \tau^{s}\right) R=q^{t s}$. The $R$-matrix satisfies all the axioms stated above and thus turns $\mathcal{Z}_{q}$ into a quasi-triangular Hopf algebra. Moreover, a ribbon element is given by $v=\sum q^{-t^{2}} P^{t}$.

It is natural to introduce a $*$-operation on $\mathcal{Z}_{q}$ such that $h^{*}=h^{-1}$. The relations (2.2) hold due to the co-commutativity of $\Delta$, i.e., $\Delta^{\prime}=\Delta$, and the property $R=R^{\prime}$. A direct computation shows that the $S$-matrix $\mathrm{S}$ is invertible only for odd integer $p$. Summarizing all this, the algebra $\mathcal{Z}_{q}, q=\exp (2 \pi i / p)$ is a semi-simple ribbon Hopf-*-algebra. It is a modular Hopf algebra for all odd integer $p$. The reader is invited to check that for $\mathcal{Z}_{q}$ the quantum trace $t r_{q}^{I}$ coincides with the standard one.

2.2 Universal elements and $R$-matrix formalism. Modular Hopf algebras admit a very elegant $R$-matrix description. For its presentation, let us introduce another (auxiliary) copy, $\mathcal{G}_{a}$, of $\mathcal{G}$ and let us consider the $R$-matrix as an object in $\mathcal{G}_{a} \otimes \mathcal{G}$. To distinguish the latter clearly from the usual $R$, we denote it by

$$
N_{+} \equiv R \in \mathcal{G}_{a} \otimes \mathcal{G}, \quad N_{-} \equiv\left(R^{\prime}\right)^{-1} \in \mathcal{G}_{a} \otimes \mathcal{G}
$$

At the same time let us introduce the standard symbols $R_{+}=R$ and $R_{-}=\left(R^{\prime}\right)^{-1} \in$ $\mathcal{G}_{a} \otimes \mathcal{G}_{a}$. Quasi-triangularity of the $R$-matrix furnishes the relations

$$
\begin{aligned}
& \Delta_{a}\left(N_{ \pm}\right)=\stackrel{1}{N} \stackrel{2}{N}_{ \pm} \quad, \quad R_{+} \stackrel{1}{N} \stackrel{2}{N}{ }_{-}=\stackrel{2}{N} \stackrel{1}{N}_{+} R_{+} \text {, } \\
& R_{+} \stackrel{1}{N} \stackrel{2}{N}_{ \pm}=\stackrel{2}{N} \stackrel{1}{N}_{ \pm} R_{+} \text {. }
\end{aligned}
$$

Here we use the same notations as in the introduction, and $\Delta_{a}\left(N_{ \pm}\right)=(\Delta \otimes i d)\left(N_{ \pm}\right) \in$ $\mathcal{G}_{a} \otimes \mathcal{G}_{a} \otimes \mathcal{G}$. The subscript ${ }_{a}$ reminds us that $\Delta_{a}$ acts on the auxiliary (i.e., first) component of $N_{ \pm}$. To be perfectly consistent, the objects $R_{ \pm}$in the preceding equations should all be equipped with a lower index $a$ to show that $R_{ \pm} \in \mathcal{G}_{a} \otimes \mathcal{G}_{a}$ etc. We hope that no confusion will arise from omitting this subscript on $R_{ \pm}$. The equations (2.5) are somewhat redundant: in fact, the exchange relations in the second line follow from the first equation in the first line. This underlines that the formula for $\Delta_{a}\left(N_{ \pm}\right)$encodes information about the product in $\mathcal{G}$ rather than the co-product.

Next, we combine $N_{+}$and $N_{-}$into one element

$$
N:=N_{+}\left(N_{-}\right)^{-1} \in \mathcal{G}_{a} \otimes \mathcal{G} .
$$

\footnotetext{
${ }^{6}$ The co-product $\Delta$ of $\mathcal{G}$ acts on $N_{ \pm}$according to $\Delta\left(N_{ \pm}\right)=(i d \otimes \Delta)\left(N_{ \pm}\right)=N_{ \pm}^{\prime} N_{ \pm}^{\prime \prime} \in \mathcal{G}_{a} \otimes \mathcal{G} \otimes \mathcal{G}$. Here $N_{ \pm}^{\prime}$ and $N_{ \pm}^{\prime \prime}$ have the unit element $e \in \mathcal{G}$ in the third and second tensor factor, respectively.
} 
From the properties of $N_{ \pm}$we obtain an expression for the action of $\Delta_{a}$ on $N$,

$$
\begin{aligned}
R_{+} \Delta_{a}(N)= & R_{+} \stackrel{1}{N} \stackrel{2}{N} \stackrel{2}{N}{ }_{-}^{-1} \stackrel{1}{N}-1=\stackrel{2}{N} \stackrel{1}{N} R_{+} R_{+} \stackrel{2}{N_{-}^{-1}} \stackrel{1}{N_{-}^{-1}}= \\
& =\stackrel{2}{N} \stackrel{2}{N}_{-}^{-1} R_{+} \stackrel{1}{N} \stackrel{1}{N}_{-}^{-1}=\stackrel{2}{N} R_{+} \stackrel{1}{N} .
\end{aligned}
$$

As seen above, the formula for $\Delta_{a}(N)$ encodes relations in the algebra $\mathcal{G}$ and implies, in particular, the following exchange relations for $N$ :

$$
R_{-}^{-1} \stackrel{2}{N} R_{+} \stackrel{1}{N}=R_{-}^{-1} R_{+} \Delta_{a}(N)=R_{-}^{-1} \Delta_{a}^{\prime}(N) R_{+}=\stackrel{1}{N} R_{-}^{-1} \stackrel{2}{N} R_{+} .
$$

This kind of relations appeared first in [47] to describe relations in $U_{q}(\mathfrak{G})$. One may in fact also go in the other direction, which means to reconstruct a modular Hopf algebra $\mathcal{G}$ from an object $N$ satisfying the above exchange relations. To begin with, one has to choose linear maps $\pi: \mathcal{G}_{a} \mapsto \mathbb{C}$ in the dual $\mathcal{G}_{a}^{\prime}$ of $\mathcal{G}_{a}$. When such linear forms $\pi \in \mathcal{G}_{a}^{\prime}$ act on the first tensor factor of $N \in \mathcal{G}_{a} \otimes \mathcal{G}$ they produce elements in $\mathcal{G}$ :

$$
\pi(N) \equiv(\pi \otimes i d)(N) \quad \in \mathcal{G} \text { for all } \pi \in \mathcal{G}_{a}^{\prime}
$$

$\pi(N) \in \mathcal{G}$ will be called the $\pi$-component of $N$ or just component of $N$. It has been shown in [9] that the components of $N$ generate the algebra $\mathcal{G}$, that is, one can reconstruct the modular Hopf algebra $\mathcal{G}$ from the object $N$. A more precise formulation is given by the following lemma.

Lemma 1 [9] Let $\mathcal{G}_{a}$ be a finite-dimensional, semi-simple modular Hopf algebra and $\mathcal{N}$ be the algebra generated by components of $N \in \mathcal{G}_{a} \otimes \mathcal{N}$ subject to the relations

$$
\stackrel{1}{N} R_{+} \stackrel{2}{N}=R_{+} \Delta_{a}(N)
$$

where we use the same notations as above. Then $N$ can be decomposed into a product of elements $N_{ \pm} \in \mathcal{G}_{a} \otimes \mathcal{N}, N=N_{+} N_{-}^{-1}$, such that

$$
\begin{gathered}
\Delta(N) \equiv N_{+}^{\prime} N^{\prime \prime}\left(N_{-}^{\prime}\right)^{-1} \quad \in \mathcal{G}_{a} \otimes \mathcal{N} \otimes \mathcal{N} \\
\epsilon\left(N_{ \pm}\right) \equiv e \in \mathcal{G}_{a} \quad, \quad \mathcal{S}\left(N_{ \pm}\right) \equiv N_{ \pm}^{-1} \in \mathcal{G}_{a} \otimes \mathcal{N} \quad, \quad N_{ \pm}^{*} \equiv N_{\mp}
\end{gathered}
$$

define a Hopf-algebra structure on $\mathcal{N}$. Here, the action of $\Delta, \epsilon, \mathcal{S}$ on the second tensor component of $N, N_{ \pm}$is understood. $N_{+}^{\prime}, N_{-}^{\prime}$ and $N^{\prime \prime}$ are regarded as elements of $\mathcal{G}_{a} \otimes \mathcal{G} \otimes \mathcal{G}$ with trivial entry in the third and in the second tensor factors, respectively. As a Hopf algebra, $\mathcal{N}$ is isomorphic to $\mathcal{G}_{a}$.

There is another object, similar to $N$, that is equally natural to consider and that will appear later in the text,

$$
\widetilde{N}:=N_{+}^{-1} N_{-} \in \mathcal{G}_{a} \otimes \mathcal{G} .
$$

Its properties are derived in complete analogy with our treatment of $N$,

$$
R_{-} \Delta_{a}(\widetilde{N})=\stackrel{1}{\widetilde{N}} R_{-} \stackrel{2}{\widetilde{N}}, \quad R_{+}^{-1} \stackrel{1}{\widetilde{N}} R_{-} \stackrel{2}{\widetilde{N}}=\stackrel{2}{\widetilde{N}} R_{+}^{-1} \stackrel{1}{\widetilde{N}} R_{-}
$$

An appropriate version of Lemma 1 establishes an isomorphism between the algebra $\widetilde{\mathcal{N}}$ generated by components of $\widetilde{N}$ and the algebra $\mathcal{G}_{o p}$. The latter stands for the quantum algebra $\mathcal{G}$ with opposite multiplication, i.e., elements $\xi, \eta \in \mathcal{G}_{o p}$ are multiplied according to $\xi \cdot \eta:=\eta \xi$. 
Observe that property (2.2) implies that $N^{*}=\tilde{N}$. We wish to rewrite this simple formula for the action of $*$ on $N$ in a more sophisticated way which proves to be useful in the sequel. For this purpose, let us introduce an element $S \in \mathcal{G}_{a} \otimes \mathcal{G}$ as follows

$$
S:=N_{+} \Delta(\kappa)(\kappa \otimes \kappa)^{-1}=N_{-} \Delta\left(\kappa^{-1}\right)(\kappa \otimes \kappa),
$$

where $\kappa$ is some central square root of the ribbon element $v \in \mathcal{G}$, i.e., $\kappa^{2}=v$ and $\kappa$ commutes with all $\xi \in \mathcal{G}$. The two expressions for $S$ given in 2.10 are equivalent due to (2.1). It is easy to check that

$$
S^{*}=S, \quad S^{\prime} \equiv P S P=S^{-1} .
$$

Now we are able to rewrite the $*$-operation on $N$ and $\widetilde{N}$ with the help of $S$ :

$$
N^{*}=S^{-1} N^{-1} S, \quad \tilde{N}^{*}=S \tilde{N}^{-1} S^{-1}
$$

ExAmple: (The universal elements for $\mathcal{Z}_{q}$ ) The notion of universal elements can be illustrated with the example of $\mathcal{Z}_{q}$. The elements $N_{ \pm} \in \mathcal{G}_{a} \otimes \mathcal{G}$ are constructed from the $R$-matrix (2.4): $N_{ \pm}=\sum_{t . s} q^{ \pm t s} P^{t} \otimes P^{s}=\sum_{s} P^{s} \otimes h^{ \pm s}$ and hence $N=\sum_{s} P^{s} \otimes h^{2 s}$. The functorial properties $(2.5),(2.8)$ can be verified by using the obvious identity $\Delta\left(P^{s}\right)=$ $\sum_{k} P^{k} \otimes P^{s-k}$. In order to make these properties more transparent, we introduce an Hermitian operator $\widehat{p}$ such that $h=q^{\widehat{p}}$. It follows from the definition of $\tau^{s}$ that $\tau^{s}(\widehat{p})=s$ and that the co-product, antipode and co-unit act on $\widehat{p}$ according to

$$
\Delta(\widehat{p})=\widehat{p} \otimes e+e \otimes \widehat{p}, \quad \mathcal{S}(\widehat{p})=-\widehat{p}, \quad \epsilon(\widehat{p})=0 .
$$

In these notations, the characteristic projector (2.3) acquires the form $P^{s}=\frac{1}{p} \sum_{m} q^{m(\widehat{p}-s)}$ and the universal elements $N_{ \pm}, N, \widetilde{N}$ are given by

$$
N_{ \pm}=q^{ \pm \hat{p} \otimes \widehat{p}}, \quad N=q^{2 \widehat{p} \otimes \widehat{p}}, \quad \widetilde{N}=q^{-2 \widehat{p} \otimes \widehat{p}}
$$

These expressions simplify the task of checking the functoriality relations in (2.5),

$$
\Delta_{a}\left(N_{ \pm}\right)=q^{ \pm(\Delta \otimes i d) \hat{p} \otimes \widehat{p}}=q^{ \pm(\widehat{p} \otimes e+e \otimes \widehat{p}) \otimes \widehat{p}}=\stackrel{1}{N} \pm \stackrel{2}{N} \pm
$$

Observe that the ribbon element $v=\sum_{s} q^{-s^{2}} P^{s}$ can be written as $v=q^{-\widehat{p}^{2}}$ and hence we may choose $\kappa=q^{-\frac{1}{2} \widehat{p}^{2}}$. A simple calculation gives $S=e \otimes e$ for the element $S$ defined in (2.10). Thus, formulae (2.12) simplify for $\mathcal{Z}_{q}$ and become $N^{*}=N^{-1}$ and $\widetilde{N}^{*}=\widetilde{N}^{-1}$.

2.3 Vertex operators and their structure data. Our next aim is to recall the theory of tensor operators for a semi-simple modular Hopf algebra $\mathcal{G}$. To this end, we combine the carrier spaces $V^{I}$ of its finite dimensional irreducible *-representations $\tau^{I}$ into the model space $\mathcal{M}=\oplus_{I} V^{I}$. Each subspace $V^{I} \subset \mathcal{M}$ appears with multiplicity one. The model space $\mathcal{M}$ comes equipped with a canonical action of our modular Hopf algebra so that we can think of $\mathcal{G}$ as being contained in the associative algebra $\mathcal{V}=\operatorname{End}(\mathcal{M})$ of endomorphisms on $\mathcal{M}$. Let us also introduce $\mathcal{C} \subset \mathcal{V}$ to denote the center of $\mathcal{G} \subset \mathcal{V}$ and e for the unit element of $\mathcal{V}$.

Definition 1 (Vertex operator) An invertible element $\Phi \in \mathcal{G}_{a} \otimes \mathcal{V}$ is called a vertex operator for $\mathcal{G}$, if 
1. $\Phi$ intertwines the action of $\mathcal{G}$ on the model space $\mathcal{M}$ in the sense that

$$
\xi \Phi=\Phi \Delta^{\prime}(\xi) \quad \text { for all } \xi \in \mathcal{G} .
$$

Here $\xi=e \otimes \xi$ on the l.h.s. and $\Delta^{\prime}(\xi)=P \Delta(\xi) P$ on the r.h.s. are both regarded as elements in $\mathcal{G}_{a} \otimes \mathcal{V}$.

2. $\Phi$ obeys the following generalized unitarity relation

$$
\Phi^{*}=S^{-1} \Phi^{-1}=\kappa_{a} \kappa N_{+}^{-1} \Phi^{-1} \kappa^{-1},
$$

where $S \in \mathcal{G}_{a} \otimes \mathcal{G}$ was defined in (2.10)-(2.11). On the r.h.s., $\kappa^{ \pm 1}=\left(e \otimes \kappa^{ \pm 1}\right)$ and $\kappa_{a}=(\kappa \otimes \mathrm{e})$, so that all these factors are elements of $\mathcal{G}_{a} \otimes \mathcal{V}$.

Invertibility of $\Phi$ means that there exists an element $\Phi^{-1} \in \mathcal{G}_{a} \otimes \mathcal{V}$ such that $\Phi \Phi^{-1}=$ $e \otimes \mathrm{e}=\Phi^{-1} \Phi$.

Since Definition 1 is fundamental to what follows below, let us discuss it in more detail. In eqs. (2.14)-(2.15) it would be possible to replace $\Delta^{\prime}$ by $\Delta$ and at the same time $S$ by $S^{-1}$. We shall meet elements $\Phi$ with such properties later and call them vertex operators as well.

The relation (2.14) describes the covariance property of $\Phi$. It means that $\Phi$ is a universal tensor operator for $\mathcal{G}$ (see, e.g., 443]). More precisely, we may evaluate the element $\Phi \in \mathcal{G}_{a} \otimes \mathcal{V}$ with representations $\tau^{I}$ of $\mathcal{G}$ to obtain matrices $\Phi^{I}=\left(\tau^{I} \otimes i d\right)(\Phi) \in \operatorname{End}\left(V^{I}\right) \otimes$ $\mathcal{V}$. The rows of these matrices form tensor operators which transform covariantly according to the representation $\tau^{I}$ of $\mathcal{G}$. The relation (2.14) may be rewritten in the $R$-matrix formalism of Subsection 2.2 (see [6], where a similar calculation was discussed) as follows:

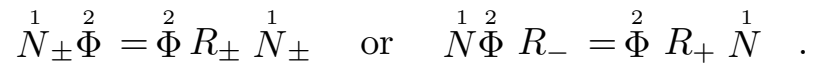

These relations are equivalent [17] to the definition of deformed tensor operators in terms of generalized adjoint actions of $\mathcal{G}$ which is often used in the theory of ( $q$-deformed) tensor operators (see, e.g., [15]).

Our formula (2.15) for the $*$-operation on $\Phi$ certainly deserves a more detailed explanation.] Both expressions we have provided describe $\Phi^{*}$ in terms of $\Phi^{-1}$. Using the intertwining relation (2.14) one concludes that the conjugated vertex operator obeys a transformation law which differs from the covariance properties of the inverse $\Phi^{-1}$ :

$$
\Phi^{-1} \xi=\Delta^{\prime}(\xi) \Phi \quad \text { while } \quad \Phi^{*} \xi=\Delta(\xi) \Phi^{*}
$$

The second relation follows from our assumption (2.2) on the behaviour of the co-product under conjugation. Comparison of the two transformation laws motivates to multiply $\Phi^{-1}$ with a factor $N_{+}^{-1}$ so that we obtain two objects with identical covariance properties, namely $\Phi^{*}$ and $N_{+}^{-1} \Phi^{-1}$. In addition, the operation $*$ is supposed to be an involution, i.e., $\left(\Phi^{*}\right)^{*}=\Phi$. This requires to dress the operator $N_{+}^{-1} \Phi^{-1}$ with factors of $\kappa$ as we did in the second expression for $\Phi^{*}$ in $(2.15)$. All these factors can be moved to the left of $\Phi^{-1}$ with the help of eq. (2.14), so that $\Phi^{*}=S^{-1} \Phi^{-1}$. The identity $\left(\Phi^{*}\right)^{*}=\Phi$ holds then as a consequence of (2.11).

\footnotetext{
${ }^{7}$-operations of a similar form have appeared in $\left.43,3,6\right]$.
} 
Suppose for the moment that we are given a vertex operator $\Phi$ in the sense of our Definition 1. Then we can use it to construct the following structure data of $\Phi$,

$$
\begin{aligned}
F & :=\stackrel{2}{\Phi} \stackrel{1}{\Phi} \Delta_{a}\left(\Phi^{-1}\right) \in \mathcal{G}_{a} \otimes \mathcal{G}_{a} \otimes \mathcal{V}, \\
\sigma(\mathrm{f}) & :=\Phi(e \otimes \mathrm{f}) \Phi^{-1} \text { for all } \mathrm{f} \in \mathcal{C} \subset \mathcal{V}, \\
D & :=\Phi N \Phi^{-1} \in \mathcal{G}_{a} \otimes \mathcal{V} .
\end{aligned}
$$

As they are defined, the last tensor components of $F, D$ and $\sigma(f)$ belong to the algebra $\mathcal{V}$. However, with the help of relation $(2.14)$ and standard axioms of Hopf algebra it is easy to see that $F, D$ and $\sigma(\mathrm{f})$ commute with all elements $\xi \in \mathcal{G} \subset \mathcal{V}$ and hence that $F \in \mathcal{G}_{a} \otimes \mathcal{G}_{a} \otimes \mathcal{C}$ while $\sigma(\mathrm{f}), D \in \mathcal{G}_{a} \otimes \mathcal{C}$. Before we give a comprehensive list of properties of the structure data, we introduce some more notations,

$$
\begin{aligned}
\mathcal{R}_{ \pm} & \equiv F^{\prime} R_{ \pm} F^{-1} \in \mathcal{G}_{a} \otimes \mathcal{G}_{a} \otimes \mathcal{C} \text { and } \\
\Delta_{F}(\xi) & \equiv F(\Delta(\xi) \otimes \mathrm{e}) F^{-1} \in \mathcal{G}_{a} \otimes \mathcal{G}_{a} \otimes \mathcal{C} .
\end{aligned}
$$

Here $F^{\prime}=(P \otimes \mathrm{e}) F(P \otimes \mathrm{e})$. As a consequence of eqs. (2.17)-(2.18) and our definition $(2.20)$ we obtain the following exchange relations for vertex operators:

$$
\mathcal{R}_{ \pm} \stackrel{2}{\Phi} \stackrel{1}{\Phi}=\stackrel{1}{\Phi} \stackrel{2}{\Phi} R_{ \pm}, \quad \mathcal{R}_{ \pm} \stackrel{2}{\sigma} \stackrel{1}{\sigma}(\mathrm{f})=\stackrel{1}{\sigma} \stackrel{2}{\sigma}(\mathrm{f}) \mathcal{R}_{ \pm}
$$

It is also worth noticing that one may think of $\mathcal{R}$ and $\Delta_{F}$ as being obtained from $R$ and $\Delta$ through a twist with $F$ in the sense of Drinfeld [25].

Proposition 1 (Properties of the structure data) Let the structure data be defined as in eqs. (2.17)-(2.19). Then it follows from Definition 1 that

1. the element $D \in \mathcal{G}_{a} \otimes \mathcal{C}$ may be expressed in terms of $\sigma$ and the ribbon element $v$ so that

$$
D=v_{a} \mathrm{v}^{-1} \sigma(\mathrm{v}) \text {. }
$$

Here $v_{a}=(v \otimes \mathrm{e}) \in \mathcal{G}_{a} \otimes \mathcal{C}$ and $\mathrm{v}=(e \otimes \mathrm{v}) \in \mathcal{G}_{a} \otimes \mathcal{C}$, that is, we denote the ribbon element by $v_{a}$ and $\vee$ when it is regarded as an element of $\mathcal{G}_{a}$ or $\mathcal{C}$, respectively.

2. the elements $F, \mathcal{R}_{ \pm} \in \mathcal{G}_{a} \otimes \mathcal{G}_{a} \otimes \mathcal{C}$ and $D \in \mathcal{G}_{a} \otimes \mathcal{C}$ together with the homomorphism $\sigma: \mathcal{C} \rightarrow \mathcal{G}_{a} \otimes \mathcal{C}$ obey the following set of relations:

$$
\begin{array}{cc}
(e \otimes F)\left(\left(i d \otimes \Delta_{a}\right)(F)\right)=\stackrel{3}{\sigma}(F)\left(\left(\Delta_{a} \otimes i d\right)(F)\right), \\
\stackrel{1}{D} \mathcal{R}_{-}=\mathcal{R}_{+} \stackrel{2}{\sigma}(D) & , \quad \mathcal{R}_{-} \stackrel{2}{D}=\stackrel{1}{\sigma}(D) \mathcal{R}_{+}, \\
\stackrel{2}{\sigma} \stackrel{1}{\sigma}(\mathfrak{f})=\Delta_{F}(\sigma(\mathfrak{f})) & \text { for all } \mathrm{f} \in \mathcal{C}, \\
\mathcal{R}_{ \pm, 12} \stackrel{2}{\sigma}\left(\mathcal{R}_{ \pm, 13}\right) \mathcal{R}_{ \pm, 23}=\stackrel{1}{\sigma}\left(\mathcal{R}_{ \pm, 23}\right) \mathcal{R}_{ \pm, 13} \stackrel{3}{\sigma}\left(\mathcal{R}_{ \pm, 12}\right) .
\end{array}
$$

The symbol $\stackrel{2}{\sigma}(D)$ denotes $(i d \otimes \sigma)(D) \in \mathcal{G}_{a} \otimes \mathcal{G}_{a} \otimes \mathcal{C}$ and $\stackrel{1}{\sigma}(D)=(P \otimes \mathrm{e}) \stackrel{2}{\sigma}(D)(P \otimes \mathrm{e})$ with $P$ being the permutation. Similar conventions apply to eqs. (2.24), (2.2X).

3. the behaviour of the structure data with respect to the *-operation is given by

$$
\begin{array}{ccl}
F^{*}=S_{a} F^{-1} & \text { with } & S_{a}=\left(R_{+} \Delta(\kappa)(\kappa \otimes \kappa)^{-1}\right) \otimes \mathrm{e} \in \mathcal{G}_{a} \otimes \mathcal{G}_{a} \otimes \mathcal{C}, \\
\mathcal{R}_{ \pm}^{*}=\mathcal{R}_{ \pm}^{-1} & , & D^{*}=D^{-1}, \\
\sigma(\mathrm{f})^{*}=\sigma\left(\mathrm{f}^{*}\right) & , & \left(\Delta_{F}(\xi)\right)^{*}=\Delta_{F}\left(\xi^{*}\right),
\end{array}
$$


for all $\xi \in \mathcal{G}$ and $\mathrm{f} \in \mathcal{C}$. It means, in particular, that $D, \mathcal{R}_{ \pm}$are unitary while $\sigma, \Delta_{F}$ act as $*$-homomorphisms.

A proof of the main statements can be found in Appendix A.1. It should be mentioned that some of the relations given in Proposition 1 have appeared in the literature before. The equation (2.27) is probably the most characteristic in our list as it generalizes the usual Yang-Baxter equation. It appeared first in connection with the quantum Liouville model [37]; later some universal solution for equation (2.27) in the case of $\mathcal{G}=U_{q}(s l(2))$ has been found [11]. More recently in [12], the elements $F$ and $\mathcal{R}$ and their relations were reinterpreted in the language of quasi-Hopf algebras [25]. As we remarked already, $F$ may be regarded as a twist and it follows from eq. (2.24) that the twisted co-product $\Delta_{F}$ is quasi-coassociative with co-associator $\phi=\stackrel{3}{\sigma}\left(F_{12}\right) F_{12}^{-1}$. The latter can be used to rewrite eq. (2.27) as a quasi Yang-Baxter equation (more details are discussed, e.g., in [12, 18]).

Relations (2.19), (2.25) and the first equation in (2.22) have been introduced in [2] in a description of deformed cotangent bundles $T_{q}^{*} G$. There, an object $N$ was defined in terms of $\Phi$ and $D$ through eq. (2.19). The relation (2.25) allowed to derive exchange relations for $N$ which guaranteed that coordinate functions for the fibers of $T_{q}^{*} G$ could be obtained from $N$.

We shall see later that the equations in Proposition 1 have a number of important implications for the lattice theories. Reversing this logic, many of the relations in Proposition 11 were conjectured as natural properties of a coordinate dependent braiding matrix in the continuum WZNW-model [26, 20, 21].

2.4 Gauge transformations of vertex operators. There exists a large gauge freedom in the choice of vertex operators $\Phi$. In fact, one may replace $\Phi \mapsto \Lambda \Phi$ with $\Lambda \in \mathcal{G}_{a} \otimes \mathcal{C}$ being invertible and unitary. This transformation does not change the general properties (2.14)-(2.15) of vertex operators but certainly effects their structure data. Namely, after the action of $\Lambda$ on $\Phi$ the initial structure data transform into following ones:

$$
\begin{gathered}
F \mapsto \stackrel{2}{\Lambda} \stackrel{2}{\sigma}(\Lambda) F \Delta_{a}\left(\Lambda^{-1}\right) \quad, \quad D \mapsto \Lambda D \Lambda^{-1} \\
\sigma(\mathrm{f}) \mapsto \Lambda \sigma(\mathrm{f}) \Lambda^{-1}
\end{gathered}
$$

where $\stackrel{2}{\sigma}(\Lambda)=(i d \otimes \sigma)(\Lambda) \in \mathcal{G}_{a} \otimes \mathcal{G}_{a} \otimes \mathcal{C}$, as before. One may reduce such a gauge freedom by additional requirements on the structure data or on the vertex operators. For instance, the gauge freedom allows to normalize the vertex operators in the following sense. Consider the element $\mathrm{w}:=\epsilon_{a}(\Phi) \equiv(\epsilon \otimes i d) \Phi \in \mathcal{V}$, where $\epsilon: \mathcal{G} \mapsto \mathbb{C}$ stands for the co-unit of $\mathcal{G}$. An application of the Hopf algebra axiom $(\epsilon \otimes i d) \Delta=i d$ to (2.14) furnishes the identity $\xi \mathrm{w}=\mathrm{w} \xi$ and hence $\mathrm{w} \in \mathcal{C}$. From this and eqs. 2.18)-(2.19) we conclude that

$$
(\epsilon \otimes i d) D=\mathrm{e} \quad, \quad(\epsilon \otimes i d) \sigma(\mathrm{f})=\mathrm{f} \text { for all } \mathrm{f} \in \mathcal{C} .
$$

Moreover, (2.15) implies unitarity of $\mathrm{w}$ (observe that $(\epsilon \otimes i d)(S)=e$ ). Therefore, we can perform the gauge transformation $\Phi \mapsto\left(e \otimes \mathrm{w}^{-1}\right) \Phi$, which does not change $\sigma$ and $D$ but normalizes $F$ and $\Phi$ so that, without loss of generality, we may assume

$$
\epsilon_{a}(\Phi)=\mathrm{e} \in \mathcal{C}, \quad(i d \otimes \epsilon \otimes i d) F=(\epsilon \otimes i d \otimes i d) F=e \otimes \mathrm{e} \in \mathcal{G}_{a} \otimes \mathcal{C}
$$

The normalization of $F$ follows from the normalization and operator product expansion of $\Phi$ with the help of $(\epsilon \otimes i d) \Delta=i d=(i d \otimes \epsilon) \Delta$. It also leads to the identities $(\epsilon \otimes i d) \mathcal{R}_{ \pm}=(i d \otimes \epsilon) \mathcal{R}_{ \pm}=e \otimes \mathrm{e}$ 
Finally, let us notice that multiplication of vertex operators $\Phi$ by element $\mathrm{F} \in \mathcal{G}_{a} \otimes \mathcal{G}$ from the right, i.e., $\Phi \mapsto \Phi \mathrm{F}$, corresponds to twisting the co-product of $\mathcal{G}$. \$ Transformations of this kind relate vertex operators $\Phi_{q}=\Phi_{1} \mathrm{~F}_{q}$ for the deformed universal enveloping algebras $U_{q}(\mathfrak{G})$ with unitary vertex operators $\Phi_{1}$ of the undeformed algebras $U(\mathfrak{G})$ [25].

2.5 On the construction of vertex operators. So far, we have considered the vertex operators as given objects. In the spirit of Lemma 1, however, we can reverse our approach and think of them as being defined through eqs. (2.17)-(2.19) with an appropriate set of structure data. This is made more precise in the following proposition.

Proposition 2 (Reconstruction of $\Phi$ from structure data) For a modular Hopf algebra $\mathcal{G} \cong \mathcal{G}_{a}$ with center $\mathcal{C}$, let $F \in \mathcal{G}_{a} \otimes \mathcal{G}_{a} \otimes \mathcal{C}$ and a homomorphism $\sigma: \mathcal{C} \rightarrow \mathcal{G}_{a} \otimes \mathcal{C}$ be given. Define the elements $D \in \mathcal{G}_{a} \otimes \mathcal{C}, \mathcal{R}_{ \pm} \in \mathcal{G}_{a} \otimes \mathcal{G}_{a} \otimes \mathcal{C}$ through equations (2.23), (2.20), respectively, and suppose that $F, \sigma$ (together with $D, \mathcal{R}_{ \pm}$) satisfy the relations (2.24)-(2.28). Then there exists a vertex operator $\Phi \in \mathcal{G}_{a} \otimes \mathcal{V}$ for $\mathcal{G}$ such that

$$
\stackrel{2}{\Phi} \stackrel{1}{\Phi}=F \Delta_{a}(\Phi) \quad, \quad \Phi \mathrm{f}=\sigma(\mathrm{f}) \Phi .
$$

In particular, the invertible element $\Phi \in \mathcal{G}_{a} \otimes \mathcal{V}$ has the properties (2.14)-(2.15) and the algebra $\mathcal{V}$ generated by its components is associative. $\mathcal{V}$ may be identified with the algebra of operators on the model space $\mathcal{M}=\bigoplus_{I} V^{I}$, as before.

Proof: Let us only sketch the proof since it is based on the same computations that are involved in the proof of Proposition 11. The construction of $\Phi$ starts from eqs. (2.29). In fact, one can use them to build an abstract algebra $\widetilde{\mathcal{V}}$ which is generated by components of an object $\Phi \in \mathcal{G}_{a} \otimes \widetilde{\mathcal{V}}$ and elements in $\mathcal{C}$ such that the two relations (2.29) hold. The properties (2.24), (2.26) ensure this algebra to be well defined and associative. Due to eqs. (2.28), $\mathcal{V}$ admits a consistent $*$-operation which makes $\Phi$ unitary in the sense of eq. (2.15). In the next step, an element $N \in \mathcal{G}_{a} \otimes \widetilde{\mathcal{V}}$ is defined by eq. (2.19). With the help of eqs. (2.25) one proves that $N$ obeys the relations (2.8), (2.16) and hence that $\widetilde{\mathcal{V}}$ contains $\mathcal{G}$ as a subalgebra. This subalgebra is finally used to analyze a concrete representation of $\widetilde{\mathcal{V}}$ and to show that $\widetilde{\mathcal{V}} \cong \mathcal{V}=\operatorname{End}(\mathcal{M})$; hence, components of $\Phi$ become operators on the model space $\mathcal{M}$.

Let us apply Proposition 2 to the example of $\mathcal{G} \cong U_{q}(\mathfrak{G})$. To this end we need to define appropriate candidates for $F$ and $\sigma$ which is achieved with the help of the Clebsch-Gordan maps $C[T L \mid S]: V^{T} \otimes V^{L} \rightarrow V^{S}$ and the $6 j$-symbols $\{: \ldots\}$ of $U_{q}(\mathfrak{G})$. Within the space $V^{L}$ of highest weight $L$, we fix a basis of eigenvectors $e_{\lambda}^{L}$ for the Cartan subalgebra with eigenvalues $\lambda$ and denote the associated Clebsch-Gordan coefficients by $\left[\begin{array}{lll}T & L & S \\ \vartheta & \lambda & \varsigma\end{array}\right]$. Now define $F, \sigma$ such that

$$
\begin{aligned}
& F^{T L}=\left(\tau^{T} \otimes \tau^{L}\right)(F) \quad \text { and } \quad \sigma^{L}(\widehat{\mathbf{p}})=\left(\tau^{L} \otimes i d\right)(\sigma(\widehat{\mathbf{p}})) \quad \text { have matrix elements } \\
& F_{\vartheta \lambda, \vartheta^{\prime} \lambda^{\prime}}^{T L}=\sum_{S, \varsigma}\left\{\begin{array}{lll}
T & L & S \\
\hat{\mathrm{p}} & \hat{\mathrm{p}}+\vartheta+\lambda & S \\
\hat{\mathrm{p}}+\lambda
\end{array}\right\}^{*}\left[\begin{array}{ccc}
T & L & S \\
\vartheta^{\prime} & \lambda^{\prime} & S
\end{array}\right] \\
& \sigma^{L}(\widehat{\mathrm{p}})_{\lambda, \lambda^{\prime}}=(\widehat{\mathrm{p}}+\lambda) \delta_{\lambda, \lambda^{\prime}}
\end{aligned}
$$

Here $\widehat{\mathrm{p}}$ is a $\operatorname{rank}(\mathfrak{G})$-dimensional vector of elements in $\mathcal{C}$ with $\tau^{K}(\widehat{\mathrm{p}})=K$. Other notations and conventions are explained in Appendix A.2.

\footnotetext{
${ }^{8}$ The object $\mathrm{F}$ should not be confused with our $F \in \mathcal{G}_{a} \otimes \mathcal{G}_{a} \otimes \mathcal{C}$. We use similar letters mainly for historical reasons.
} 
Proposition 3 (Vertex operators for $U_{q}(\mathfrak{G})$ ) There exist vertex operators $\Phi_{q}$ for the deformed universal enveloping algebras $U_{q}(\mathfrak{G})$ such that

$$
\stackrel{2}{\Phi}_{q} \stackrel{1}{\Phi}_{q}=F \Delta_{a}\left(\Phi_{q}\right) \quad, \quad \Phi_{q} \mathrm{f}=\sigma(\mathrm{f}) \Phi_{q} .
$$

Here $F$ is built up from the $6 j$-symbols and the Clebsch-Gordan maps of $U_{q}(\mathfrak{G})$ as in eq. (2.30) and $\sigma$ is given by (2.31).

The statement follows directly from the Proposition 2 once the relations (2.24) $-(2.28)$ have been checked to hold for $F, \sigma$. The latter is done in Appendix A.2. Let us mention that formulae similar to (2.30) were considered in [12, 18].

2.6 Vertex operators for $\mathcal{Z}_{q}$. To conclude our discussion of vertex operators, let us provide an explicit formula for $\Phi$ in our standard example $\mathcal{G}=\mathcal{Z}_{q}$. Let us fix a set of normalized basis vectors $|s\rangle, s=0, \ldots, p-1$, for the one-dimensional carrier spaces $V^{s}$ of the representations $\tau^{s}$. They span the $p$-dimensional model space $\mathcal{M}=\bigoplus_{s} V^{s}$. On this space one can introduce a unitary operator $\widehat{Q} \in \operatorname{End}(\mathcal{M})$ by

$$
\widehat{Q}|p-1\rangle=|0\rangle \quad \text { and } \quad \widehat{Q}|s\rangle=|s+1\rangle
$$

for all $s=0, \ldots, p-2$. This operator obeys Weyl commutation relations with the generator $h \in \mathcal{Z}_{q}$, i.e., $q \widehat{Q} h=h \widehat{Q}$. With the help of $\widehat{Q}$ and the characteristic projectors $P^{s}$ introduced in Subsection 2.1 we are able to define $\Phi$ :

$$
\Phi:=\sum_{s} P^{s} \otimes \widehat{Q}^{s}=\frac{1}{p} \sum_{s, t} q^{-s t} h^{t} \otimes \widehat{Q}^{s} \in \mathcal{G}_{a} \otimes \operatorname{End}(\mathcal{M})
$$

It follows from the unitarity of $\widehat{Q}$ and the Weyl relations of $\widehat{Q}$ and $h$ that $\Phi$ obeys all the defining properties of a vertex operator (as we explained in Subsection 2.2, the element $S$ in eq. 2.15) becomes trivial for $\mathcal{G}=\mathcal{Z}_{q}$ ). One may then compute the structure data. To this end it is convenient to employ the operator $\widehat{p}$ introduced in Subsection 2.2 such that $h=q^{\widehat{p}}$. Since the commutative algebra $\mathcal{Z}_{q}$ is isomorphic to its center $\mathcal{C}$, all elements in $\mathcal{Z}_{q}$ can be regarded as elements of $\mathcal{C}$ and we use our standard notational conventions whenever we do so, in particular we shall use $\mathrm{h}=q^{\widehat{\mathrm{p}}}$ for $\mathrm{h}, \widehat{\mathrm{p}} \in \mathcal{C}$. We also introduce an anti-Hermitian operator $\widehat{\varsigma}$ by $\widehat{Q}=e^{\widehat{\varsigma}}$, so that the Weyl relations for $\widehat{Q}$ and $h$ imply $[\widehat{p}, \widehat{\varsigma}]=$ e. Within these notations our basic objects look as follows:

$$
h=q^{\widehat{p}} \in \mathcal{G}, \quad \mathrm{v}=q^{-\widehat{\mathrm{p}}^{2}} \in \mathcal{C}, \quad \Phi=e^{\widehat{p} \otimes \widehat{\varsigma}} \in \mathcal{G}_{a} \otimes \operatorname{End}(\mathcal{M}) .
$$

Now expressions for the structure data may be obtained by short computations,

$$
F=e \otimes e \otimes \mathrm{e}, \quad \sigma\left(q^{\widehat{\mathrm{p}}}\right)=h^{-1} \otimes q^{\widehat{\mathrm{p}}}=q^{-\widehat{p} \otimes \mathrm{e}+e \otimes \widehat{\mathrm{p}}}, \quad D=q^{-2\left(\widehat{p}^{2} \otimes \mathrm{e}-\widehat{p} \otimes \widehat{\mathrm{p}}\right)}
$$

and $\mathcal{R}_{ \pm}=q^{\hat{p} \otimes \widehat{p} \otimes \mathrm{e}}$.

Let us remark that, although the example of vertex operators for $\mathcal{Z}_{q}$ is fairly trivial, it nevertheless shares some features with the case of $\mathcal{G}=U_{q}(\mathfrak{G})$. Indeed, the ribbon element of $U_{q}(\mathfrak{G})$ is given by $\mathbf{v}=q^{-\widehat{p}(\hat{\mathrm{p}}+\rho)}$ [25] where $\widehat{\mathrm{p}} \in \mathcal{C}^{\otimes_{r}}$ is a $r=\operatorname{rank}(\mathfrak{G})$-dimensional vector such that $\tau^{K}(\widehat{\mathbf{p}})=K$ and $\rho$ is the sum of the positive roots. Our above formula 2.31 means that

$$
\sigma(\widehat{\mathrm{p}})=\widehat{H} \otimes \mathrm{e}+e \otimes \widehat{\mathrm{p}}, \quad D=(\chi \otimes \mathrm{e}) \cdot q^{-2 \widehat{H} \otimes \widehat{\mathrm{p}}},
$$

where $\widehat{H}$ is a vector of elements in the Cartan subalgebra such that $\widehat{H} e_{\lambda}^{L}=\lambda e_{\lambda}^{L}$ and the element $\chi \in \mathcal{G}=U_{q}(\mathfrak{G})$ can be worked out easily with the help of eq. (2.23). Such expressions, or special cases thereof, may be found in [23, 20, 2, 21, 18]). The element $F$ and the vertex operators $\Phi$ are certainly quite non-trivial for $U_{q}(\mathfrak{G})$ (for some explicit examples see [33, 22, 19, 18]). 


\section{A TOY MODEL FOR THE DISCRETE WZNW THEORY}

In the rest of this paper we shall apply the theory of modular Hopf algebras and their vertex operators to construct and investigate the lattice WZNW-model. We start with a simple toy model for which the lattice consists of only one site and one edge (see Figure 1). When we discuss the general notion of lattice current algebras in Section 4, we shall understand that they contain chiral observables $M$ (the chiral monodromies) being assigned to the edge.

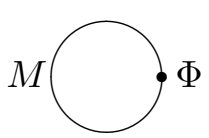

Figure 1: Single-vertex lattice. Chiral observables $M$ are assigned to the edge while chiral vertex operators $\Phi$ sit on the vertex.

3.1 Properties of chiral vertex operators. Later in the text we shall find that the global chiral observable $M$ in the lattice current algebra obeys the following relation

$$
\stackrel{2}{M} R_{+} \stackrel{1}{M}=R_{-} \Delta_{a}(M)
$$

where $R_{ \pm}, \Delta_{a}$ are attributes of the modular Hopf algebra $\mathcal{G}$ as before. Components of $M$ generate an algebra $\mathcal{J}$ with center denoted by $\mathcal{C}$.

Eq. (3.1) reminds us of the defining relation (2.6) for the universal element $N$, which contains all the information about the structure of $\mathcal{G}$. Indeed, the only difference is that the $R_{+}$on the 1.h.s. of eq. (2.6) has been replaced by $R_{-}$. A short computation reveals that we can pass from eq. (3.1) to (2.6) by rescaling $M$ with the ribbon element $v_{a}=(v \otimes e) \in \mathcal{G}_{a} \otimes \mathcal{G}$. This implies that $N \mapsto v_{a} M$ provides an isomorphism of the algebras $\mathcal{G}$ and $\mathcal{J}$. In particular, the commutation relations for $M$,

$$
R_{ \pm}^{-1} \stackrel{2}{M} R_{+} \stackrel{1}{M}=\stackrel{1}{M} R_{-}^{-1} \stackrel{2}{M} R_{\mp}
$$

coincide with eqs. (2.7) for the element $N$. The isomorphism of $\mathcal{J}$ and $\mathcal{G}$ certainly implies that there is a $*$-operation on $\mathcal{J}$ given by the formula (2.12) with $N$ replaced by $M$ (notice that the factor $v_{a}$ is unitary). The lattice theories, however, choose a different conjugation which we discuss in Subsection 3.3 below.

Now let us introduce a vertex operator $\Phi$ for $\mathcal{J} \cong \mathcal{G}$. It will be called chiral vertex operator of the toy model and its properties can be copied from the relations (2.14) - 2.19) when we keep in mind to replace $N$ by $v_{a} M$,

$$
\begin{array}{rll}
\eta \Phi=\Phi \Delta^{\prime}(\eta) & , \quad \stackrel{2}{2} R_{-}=\stackrel{2}{\Phi} R_{+} \stackrel{1}{M} \\
\stackrel{2}{\Phi} \stackrel{1}{\Phi}=F \Delta_{a}(\Phi) & , \quad & \mathcal{R}_{ \pm} \stackrel{2}{\Phi} \stackrel{1}{\Phi}=\stackrel{1}{\Phi} \stackrel{2}{\Phi} R_{ \pm}, \\
D \Phi=v_{a} \Phi M & , & \Phi \mathrm{f}=\sigma(\mathrm{f}) \Phi \text { for all } \mathrm{f} \in \mathcal{C} .
\end{array}
$$

Here $\eta \in \mathcal{J}, \mathcal{C}$ stands for the center of $\mathcal{J}$, and we used the same notations as in the previous section. The components of $\Phi \in \mathcal{G}_{a} \otimes \mathcal{V}$ give rise to the algebra $\mathcal{V}$ of chiral vertex operators. Together with components of $M$, they act on the model space $\mathcal{M}=\bigoplus_{I} V^{I}$.

We refer to the first equation in (3.4) as operator product expansions (OPE) for $\Phi$ and call $F$ the universal fusion matrix. The second formula in (3.4) follows from the operator 


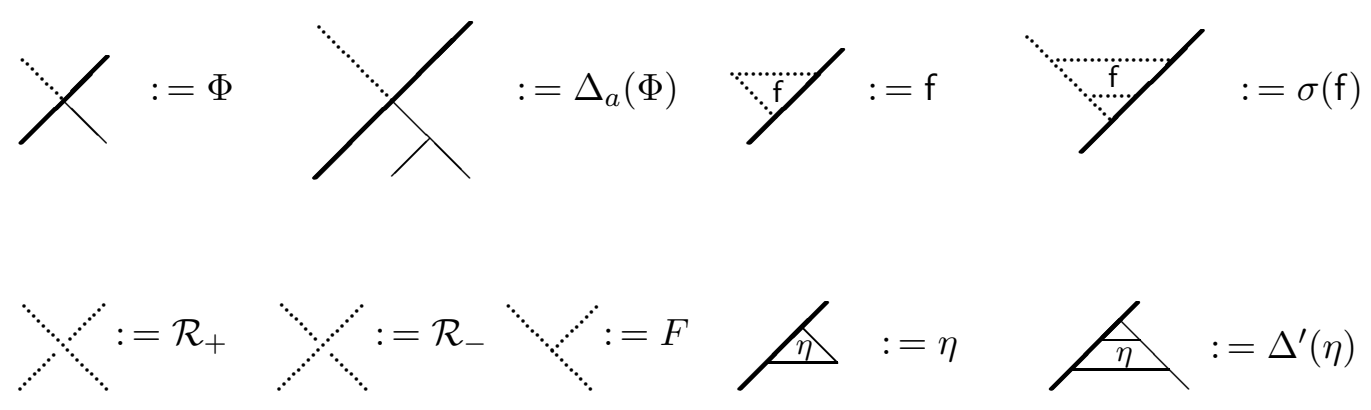

Figure 2: Graphical presentation of our basic objects. Pictures for $D$ and $M$ exist as well, but they are more complicated (cf. remarks in the text).

product expansions; it describes braid relations for the chiral vertex operators and hence leads to interpret $\mathcal{R}_{ \pm}$as the braiding matrix of our model. ?

There exists a nice pictorial presentation for the described algebraic structure. Definitions for the basic objects - except from $D, M$ - are given in Figure 2. Pictures for $M$ and $D$ may, in principle, be constructed with the help of eq. (2.23), eqs. (3.5) and an appropriate presentation of the ribbon element. From the basic blocks we can built up the equations (3.3)-(3.5) as in Figure 3. All these pictures are separated by a thick solid line into left and right halves with dotted lines appearing on the left side while thin solid lines exist only on the right side. Our graphical rules are the same as in [39], and, in their terminology, the dotted lines may be said to live in the shadow world.

3.2 Second chirality. What we have discussed so far will be relevant for right chiral objects in the discrete WZNW model. Now we have to describe an analogous construction for the left chiral sector of the theory. To distinguish the two chiralities, we mark the objects of the previous subsection by an extra index $r$ so that $M^{r}=M, \Phi^{r}=\Phi, F_{r}=$ $F, \sigma_{r}=\sigma \ldots$ etc. Their left chiral counterparts will have an index $l$.

To introduce left chiral vertex operators $\Phi^{l}$ we follow the same strategy as in the previous subsection. Namely, we postulate algebraic relations for an object $M^{l}$ (which will be justified in Section 4) and use them as the basic input for our left chiral theory. So let us assume that we are given some object $M^{l}$ such that

$$
\stackrel{1}{M}^{l} R_{-} \stackrel{2}{M}^{l}=R_{+} \Delta_{a}\left(M^{l}\right)
$$

The algebra generated by components of $M^{l}$ will be denoted by $\mathcal{J}^{l}$ and we use the symbol $\mathcal{C}^{l}$ for its center.

It is easy to see that the properties of $v_{a}^{-1} M^{l}$ coincide with those of the element $\widetilde{N}$ introduced in Subsection 2.2, eq. (2.9). This holds, in particular, for the commutation relations,

$$
R_{ \pm}^{-1} \stackrel{1}{M}^{l} R_{-} \stackrel{2}{M}^{l}=\stackrel{2}{M^{l}} R_{+}^{-1} \stackrel{1}{M^{l}} R_{\mp}
$$

Thus, the algebra $\mathcal{J}^{l}$ is isomorphic to the algebra generated by components of $\tilde{N}$, i.e., to $\mathcal{G}_{o p}$ ( op means the opposite multiplication, cf. Subsection 2.2).

\footnotetext{
${ }^{9}$ This will become clearer in the full lattice theory where braid relations of vertex operators assigned to different sites contain only $\mathcal{R}_{ \pm}$and the factor $R_{ \pm}$is absent. Observe also that in the quantum nondeformed limit, i.e., $\gamma \rightarrow 0, \hbar \neq 0, q=e^{i \hbar \gamma} \rightarrow 1$, the $R$-matrix $R_{ \pm}$approaches $e \otimes e$ whereas the limit of $\mathcal{R}_{ \pm}$is non-trivial (cf. also [2, 19]).
} 

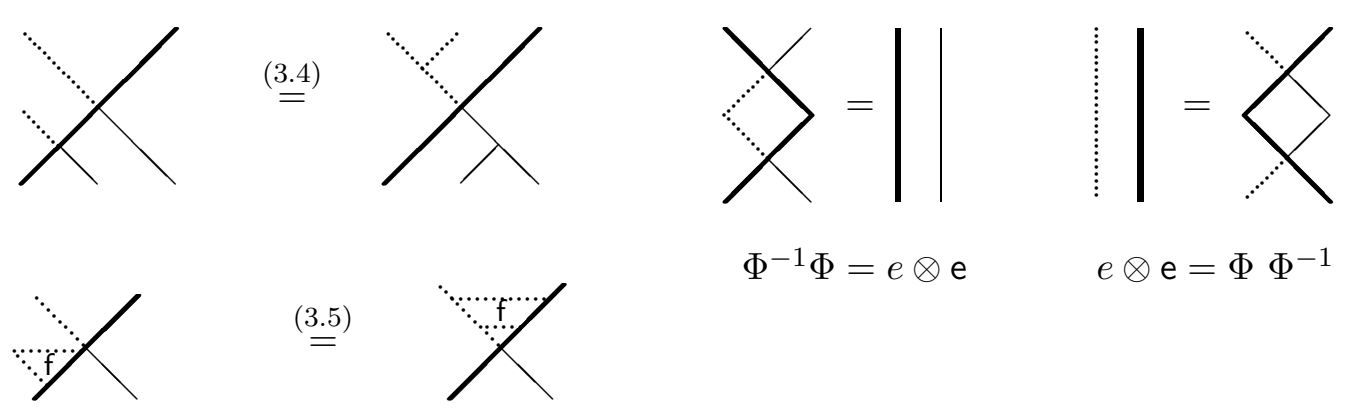

(3.5)

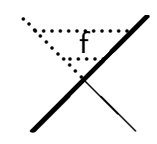

$$
\Phi^{-1} \Phi=e \otimes \mathrm{e}
$$

$e \otimes \mathrm{e}=\Phi \Phi^{-1}$
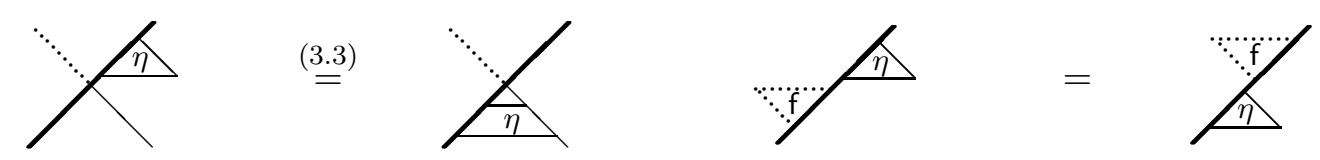

Figure 3: Pictorial presentation of some basic relations. Only the left equations in (3.3), (3.4) and the right equation in (3.5) are depicted. The figure in the lower right corner means that $f \in \mathcal{C}$ is central in $\mathcal{G}$. More rules are explained in the text.

Since eqs. (3.6)-(3.7) differ from the properties of $M^{r}$, the relations for the left chiral vertex operators will differ from those we had in the right chiral sector. The consistent definition of the left vertex operators is provided by the following list of fundamental relations:

$$
\begin{aligned}
& \eta \Phi^{l}=\Phi^{l} \Delta(\eta) \quad, \quad \stackrel{1}{\Phi}^{l} R_{-} \stackrel{2}{M}^{l}=\stackrel{2}{M}^{l} \stackrel{1}{\Phi^{l}} R_{+}, \\
& \stackrel{1}{\Phi} l \stackrel{2}{\Phi} \Phi^{l}=F_{l} \Delta_{a}\left(\Phi^{l}\right) \quad, \quad \mathcal{R}_{ \pm}^{l} \stackrel{1}{\Phi} l \stackrel{2}{\Phi} l=\stackrel{2}{\Phi}=\stackrel{1}{\Phi} l R_{ \pm}, \\
& D_{l} \Phi^{l}=v_{a}^{-1} \Phi^{l} M^{l} \quad, \quad \Phi^{l} \mathrm{f}=\sigma_{l}(\mathrm{f}) \Phi^{l} \quad \text { for all } \mathrm{f} \in \mathcal{C}^{l} \text {. }
\end{aligned}
$$

Components of $\Phi^{l} \in \mathcal{G}_{a} \otimes \mathcal{V}^{l}$ generate the algebra $\mathcal{V}^{l}$ of left chiral vertex operators and act on the left model space $\mathcal{M}^{l} \cong \bigoplus_{I} V^{I}$. Starting from the defining equation (3.6) for $M^{l}$ one may check that the exchange relations (3.8) describe a consistent transformation law of the vertex operators $\Phi^{l}$. It is then clear that the left vertex operators obey eqs. (3.9)-(3.10) with some appropriate structure data $F_{l}, \sigma_{l}, D_{l}, \mathcal{R}_{ \pm}^{l}$. The consistency relations for the left structure data can be worked out in analogy to our discussion of Proposition 1. For more detailed explanations see Appendix A.3.

Let us now combine the two chiral theories by constructing their tensor product so that all operators act on the space $\mathcal{M}^{l} \otimes \mathcal{M}^{r}$ with trivial action of the right chiral objects on the first tensor factor and vice versa. In terms of exchange relation this corresponds to

$$
\begin{aligned}
& \stackrel{1}{\Phi}^{r} \stackrel{2}{\Phi} l=\stackrel{2}{\Phi} l \stackrel{1}{\Phi}{ }^{r} \quad, \quad \stackrel{1}{M}^{r} \stackrel{2}{M}^{l}=\stackrel{2}{M}^{l} \stackrel{1}{M}^{r}, \\
& \stackrel{1}{\Phi^{r}} \stackrel{2}{M}^{l}=\stackrel{2}{M^{l}} \stackrel{1}{\Phi}^{r} \quad, \quad \stackrel{1}{\Phi} l \stackrel{2}{M}^{r}=\stackrel{2}{M}^{r} \stackrel{1}{\Phi^{l}} .
\end{aligned}
$$

Components of the chiral vertex operators $\Phi^{l}, \Phi^{r}$ generate an algebra $\mathcal{W}=\mathcal{V}^{l} \otimes \mathcal{V}^{r}$. Although this combination of chiral theories appears to be quite trivial, it sets the stage 
for the construction of the quantum group valued field $g$ that we are about to discuss in Subsection 3.4.

Before we get there, let us explain how to incorporate our new left chiral objects into the graphical presentation discussed at the end of the previous subsection. The pictures for the left chiral theory are simply mirror images of those in Figures 2,3, that is, left chiral objects have their dotted lines on the right side and thin solid lines on the left side of the thick solid line. To present the tensor product of the left- and right theory, we draw all objects into the same pictures. Now there are dotted and solid lines on both sides. If we add the rule that these lines of different style do not interfere, we obtain commutativity of the two chiralities as expressed in eqs. (3.11)-(3.12).

$3.3 *$-operation for chiral vertex operators. In principle, a $*$-operation for $M^{l}, M^{r}$ and the associated vertex operators could be introduced along the lines of Section 2. But as we indicated the lattice models choose a slightly different conjugation. Its description requires to introduce a new object.

By definition, the models spaces $\mathcal{M}^{l}, \mathcal{M}^{r}$ carry an action of the modular Hopf-algebra $\mathcal{G}$. With the help of the co-product $\Delta$ this gives rise to a canonical action of $\mathcal{G}$ on the tensor product $\mathcal{M}^{l} \otimes \mathcal{M}^{r}$ and hence to an embedding $\iota$ of the quantum algebra $\mathcal{G}$ into the algebra $\mathcal{W}=\mathcal{V}^{l} \otimes \mathcal{V}^{r}$ of chiral vertex operators. For the exchange relations of $\iota(\xi)$ and chiral vertex operators, our construction implies:

$$
\iota(\xi) \Phi^{r}=\Phi^{r} \Delta_{\iota}^{\prime}(\xi) \quad, \quad \iota(\xi) \Phi^{l}=\Phi^{l} \Delta_{\iota}(\xi)
$$

for all $\xi \in \mathcal{G}$; we used $\Delta_{\iota}(\xi)=(i d \otimes \iota) \Delta(\xi)$ and similarly for $\Delta_{\iota}^{\prime}$. These relations imply that $\Phi^{l}, \Phi^{r}$ transform covariantly with respect to our new action $\iota$ of $\mathcal{G}$ on $\mathcal{W}$. They can be rewritten in the $R$-matrix formulation,

$$
\stackrel{1}{N} \stackrel{2}{\Phi}^{r}=\stackrel{2}{\Phi} R_{ \pm} \stackrel{1}{N}{ }_{ \pm} \quad, \quad \stackrel{1}{N} \stackrel{2}{\Phi}^{l}=\stackrel{2}{=} l \stackrel{1}{\Phi} \stackrel{1}{N}_{ \pm} R_{ \pm}
$$

where $N_{ \pm}=(i d \otimes \iota)\left(R_{ \pm}\right) \in \mathcal{G}_{a} \otimes \mathcal{W}$. In our pictorial presentation the objects $N_{ \pm}$would appear as over-/under- crossings of thin and thick solid lines. Hence, they have thin solid lines on both sides of the boundary between the left and the right world. This corresponds to the fact that components of $N_{ \pm}$act nontrivially on both factors in $\mathcal{M}^{l} \otimes \mathcal{M}^{r}$, that is, they are not chiral. The same holds true for the product $N=N_{+}\left(N_{-}\right)^{-1}$.

Now we are prepared to describe the $*$-operation which is relevant for the toy model. To this end, we build an object $S_{\iota}$ with the help of $\iota$ by $S_{\iota}=(i d \otimes \iota)(S) \in \mathcal{G}_{a} \otimes \mathcal{W}$ and $S \in \mathcal{G}_{a} \otimes \mathcal{G}$ is defined as in Subsection 2.2. It is used to extend the $*$-operation on $\mathcal{G} \cong \iota(\mathcal{G}) \subset \mathcal{W}$ to the algebra of chiral vertex operators:

$$
\left(\Phi^{r}\right)^{*}=S_{\iota}^{-1}\left(\Phi^{r}\right)^{-1} \quad, \quad\left(\Phi^{l}\right)^{*}=S_{\iota}\left(\Phi^{l}\right)^{-1} .
$$

The first formula looks familiar already and since the exchange relations of $\iota(\xi)$ with $\Phi^{r}$ coincide with eq. (2.14), consistency need not to be checked again. The second formula is a variant of eq. (2.15) which is adapted to the algebraic properties of the left chiral theory. To prove that it is consistent one has to modify our discussion in Subsection 2.3 slightly. We leave this to the reader. It remains to show that the adjoints of $\Phi^{l}$ and $\Phi^{r}$ commute; this is not obvious at all, since $S_{\iota}$ is not a chiral object. Commutativity of the adjoints may be seen most easily if we rewrite the adjoints in the form (2.15) which involves conjugation with $\kappa$ (which is $\iota(\kappa)$ in our case). Then the desired consistency follows from the transformation law of vertex operators under the action of $\iota(\xi)$ and the Yang-Baxter equation (see also [6]). 
It follows from eqs. $(\overline{3.5}),(\sqrt[3.10]{ })$ that the conjugation acts on the chiral monodromies $M^{r}, M^{l}$ according to

$$
\left(M^{r}\right)^{*}=S_{\iota}^{-1}\left(M^{r}\right)^{-1} S_{\iota} \quad, \quad\left(M^{l}\right)^{*}=S_{\iota}\left(M^{l}\right)^{-1} S_{\iota}^{-1} .
$$

We shall rediscover such a behaviour for the chiral monodromies of the lattice theory in Subsection 4.3.

3.4 Quantum group valued field $g$. So far we have reached a good level of understanding for our right- and left chiral theories which act naturally on the tensor product $\mathcal{M}^{l} \otimes \mathcal{M}^{r}$ of chiral model spaces. In this subsection we would like to have a closer look at the diagonal subspace

$$
\mathcal{H}=\bigoplus_{K} V^{\bar{K}} \otimes V^{K} \subset \mathcal{M}^{l} \otimes \mathcal{M}^{r}
$$

While components of $M^{l}, M^{r}$ leave $\mathcal{H}$ invariant, this is certainly not the case for the vertex operators $\Phi^{l}, \Phi^{r}$. Nevertheless, the vertex operators can be combined into a new object $g$ which admits restriction to the diagonal subspace $\mathcal{H}$.

The construction of $g$ requires a careful preparation. Let us begin this with some remarks on the center $\mathcal{C}$ of $\mathcal{G}$ (recall that $\mathcal{C}^{r} \cong \mathcal{C}^{l} \cong \mathcal{C}$ ). First, observe that $\mathcal{C}$ is spanned by the characteristic projectors $P^{J}$ of irreducible representations $\tau^{J}$ of $\mathcal{G}$, i.e., by projectors $P^{J} \in \mathcal{C}$ which obey $\tau^{K}\left(P^{J}\right)=\delta_{K, J}$. Notice also that the antipode $\mathcal{S}$ maps the element $P^{K} \in \mathcal{C}$ to the characteristic projector $P^{\bar{K}} \in \mathcal{C}$ of the conjugate representation $\tau^{\bar{K}}$, i.e., $\mathcal{S}\left(P^{K}\right)=P^{\bar{K}} \cdot$ 四

Returning to our toy model, we combine the canonical isomorphism $\nu: \mathcal{C}^{r} \rightarrow \mathcal{C}^{l}$ and action of the antipode $\mathcal{S}$ into a map $\mathcal{S}_{l r}: \mathcal{C}^{r} \rightarrow \mathcal{C}^{l}, \mathcal{S}_{l r}(\mathrm{f})=\mathcal{S}(\nu(\mathrm{f}))$. With the help of this map we can characterize the diagonal subspace $\mathcal{H}$ as a subspace generated by all vectors $\phi \in \mathcal{M}^{l} \otimes \mathcal{M}^{r}$ such that $\mathrm{f} \phi=\mathcal{S}_{l r}(\mathrm{f}) \phi$ holds for all $\mathrm{f} \in \mathcal{C}^{r}$. In this language, the restriction to $\mathcal{H}$ means to impose the constraint $\mathrm{f}=\mathcal{S}_{l r}(\mathrm{f})$ for all $\mathrm{f} \in \mathcal{C}^{r}$. This constraint couples the two chiralities and it seems natural to restrict the choice of the left- and right structure data $F_{\alpha}, \sigma_{\alpha}, D_{\alpha}, \mathcal{R}_{ \pm}^{\alpha}$ at the same time. Notice that they were completely independent until now, as long as they solved the appropriate consistency relations. So let us agree to adjust the choice of the structure data for the left chirality to whatever we use in the right chiral part such that

$$
\begin{array}{ccc}
F_{l}=\mathcal{S}_{l r}^{(2)}\left(F_{r}^{\prime-1}\right) & D_{l}=\mathcal{S}_{l r}^{(1)}\left(D_{r}^{-1}\right) \\
\mathcal{R}_{ \pm}^{l}=\mathcal{S}_{l r}^{(2)}\left(\mathcal{R}_{ \pm}^{r \prime}\right) & , & \sigma_{l}(\mathrm{f})=\mathcal{S}_{l r}^{(1)}\left(\sigma_{r} \circ \mathcal{S}_{l r}^{-1}(\mathrm{f})\right) \\
\text { with } \quad \mathcal{S}_{l r}^{(n)}:=\left(\mathcal{S}^{-1} \otimes \mathcal{S}_{l r}^{(n-1)}\right) & \text { and } \quad \mathcal{S}_{l r}^{(0)}:=\mathcal{S}_{l r}
\end{array}
$$

and the prime on $F_{r}$ and $\mathcal{R}_{ \pm}^{r}$ denotes permutation of the first two tensor factors in $\mathcal{G}_{a} \otimes$ $\mathcal{G}_{a} \otimes \mathcal{C}$. It is not difficult to show that these formulae give consistent structure data for the left chiral theory (cf. also Appendix A.3). The motivation for eqs. (3.13), (3.14) comes from the construction of the quantum group valued field $g$. So let us define

$$
g:=\mathcal{S}_{a}\left(\Phi^{l}\right) \Phi^{r} \in \mathcal{G}_{a} \otimes \mathcal{W}
$$

\footnotetext{
${ }^{10}$ Strictly speaking, the conjugate of $\tau^{K}$ is obtained with the help of a transpose ${ }^{t}$ as ${ }^{t} \tau^{K} \circ \mathcal{S}$. The latter is isomorphic to $\tau^{\bar{K}}$ (this property defines the label $\bar{K}$ ).
} 
where $\mathcal{S}_{a}\left(\Phi^{l}\right) \equiv(\mathcal{S} \otimes i d)\left(\Phi^{l}\right)$. The element $g$ indeed preserves the constraint which we discussed above, that is

$$
\text { if } \quad \mathrm{f} g=\mathcal{S}_{l r}(\mathrm{f}) g, \text { then } g \mathrm{f}=g \mathcal{S}_{l r}(\mathrm{f}) \text { for all } \mathrm{f} \in \mathcal{C}^{r}
$$

Therefore, components of $g$ map the diagonal space $\mathcal{H}$ into itself. This remarkable property is established by a straightforward computation (see Appendix A.4).

To study properties of $g$ it is helpful to have some knowledge about the object $\mathcal{S}_{a}\left(\Phi^{l}\right)$. Simple applications of the standard Hopf algebra axioms allow to deduce

$$
\begin{gathered}
\mathcal{S}_{a}\left(\Phi^{l}\right) \xi=\Delta_{\iota}(\xi) \mathcal{S}_{a}\left(\Phi^{l}\right) \quad, \quad R_{+} \mathcal{S}_{a}\left(\stackrel{2}{\Phi}^{l}\right) \stackrel{1}{M}^{l}=\stackrel{1}{M}^{l} R_{-} \mathcal{S}_{a}\left(\stackrel{2}{\Phi}^{l}\right) \\
\mathcal{S}_{a}\left(\Phi^{l}\right)=\left(\Phi^{l}\right)^{-1} \theta_{l} \quad \text { with } \quad \theta_{l} \in \mathcal{G}_{a} \otimes \mathcal{C}^{l} .
\end{gathered}
$$

Here $\xi \in \mathcal{J}^{l} \cong \mathcal{G}$ in the first equation, $\mathcal{S}_{a}\left(\stackrel{2}{\Phi}^{l}\right)$ is a shorthand for $\left(i d \otimes \mathcal{S}_{a}\right)\left(\stackrel{2}{\Phi}^{l}\right)$, and the relation (3.19) may be regarded as a definition of $\theta_{l}$. The transformation laws of vertex operators show that $\theta_{l}$ commutes with $e \otimes \xi \in \mathcal{G}_{a} \otimes \mathcal{J}^{l}$ and hence $\theta_{l} \in \mathcal{G}_{a} \otimes \mathcal{C}^{l}$. We can actually give an explicit formula for $\theta_{l}$ in terms of $F_{l}$. If we assume for simplicity that $\epsilon_{a}\left(\Phi^{l}\right)=\mathrm{e}\left(\mathrm{cf}\right.$. Subsection 2.4), then $\theta_{l}=\sum_{\varsigma} f_{\varsigma}^{1} \mathcal{S}\left(f_{\varsigma}^{2}\right) \otimes f_{\varsigma}^{3}$, where $f_{\varsigma}^{i}$ come from the expansion $F_{l}=\sum_{\varsigma} f_{\varsigma}^{1} \otimes f_{\varsigma}^{2} \otimes f_{\varsigma}^{3}$.

Proposition 4 (Properties of $g$ ) Let $g$ denote the object defined in eq. (3.10) and restricted to the subspace $\mathcal{H}$. This element $g \in \mathcal{G}_{a} \otimes \operatorname{End}(\mathcal{H})$ obeys the following relations:

$$
\begin{aligned}
\stackrel{2}{g} \stackrel{1}{g}=\Delta_{a}(g), & R_{ \pm} \stackrel{2}{g} \stackrel{1}{g}=\stackrel{1}{g} \stackrel{2}{g} \quad R_{ \pm}, \\
M^{r} \stackrel{2}{g} \quad R_{-}=\stackrel{2}{g} \quad R_{+} M^{r}, & M^{l} R_{-} \stackrel{2}{g}=R_{+} \stackrel{2}{g} \stackrel{1}{M}^{l}, \\
M^{l} g=g M^{r}, & \vee g \mathrm{v}^{-1}=g,
\end{aligned}
$$

where in the last line $\mathrm{v}=\mathrm{v}_{r} \mathrm{v}_{l}^{-1}$ is a combination of the ribbon elements $\mathrm{v}_{r} \in \mathcal{C}^{r}$ and $\mathrm{v}_{l} \in \mathcal{C}^{l}$. Moreover, $g$ is normalized, $\epsilon_{a}(g)=\mathrm{e}$, and invertible with inverse $g^{-1}=\mathcal{S}_{a}(g)$.

Proofs of all these relations are given in Appendix A.4.

Eqs. (3.20) mean that $g$ obeys the defining relation of a quantum group $\mathcal{F}=\operatorname{Fun}_{q}(G)$. More precisely, components of $g$ generate the dual of the quantum algebra $\mathcal{G}$. The elements $M^{r}, M^{l}$ furnish algebras of left- and right-invariant vector fields for $\mathcal{F}$ and they are related to each other by means of eq. (3.22). All these equations are well known in the theory of quantum groups. In more geometric terms, they describe the deformed co-tangent bundle $T_{q}^{*} G$ [2]. [1]

Let us now explain the pictorial presentation of the object $g$ (see Figure 4). First, recall that so far left and right chiral objects lived on the same plane but on different sides of the thick solid line and there was no interaction between them. But if we want to consider the restriction from $\mathcal{M}^{l} \otimes \mathcal{M}^{r}$ to the diagonal subspace $\mathcal{H}$, we have to modify the rules. Namely, the restriction $\mathrm{f}=\mathcal{S}_{l r}(\mathrm{f})$ enforces us to change the topology by gluing the plane into a cylinder. Then we can join ends of dotted lines from both sides of the thick solid line and thus combine objects of different chirality. This is demonstrated by the graphical presentation of $g$ in Figure 4 (the dashed line continues the dotted line around the back side of cylinder and, hence, cannot interfere with any line on the front side). Figure 4 also sketches the proof of the operator product expansions of $g$ in eq. (3.20).

\footnotetext{
${ }^{11}$ Similarly, the algebra generated by components of $\Phi^{r}, M^{r}$ only, is a deformation of the co-tangent bundle $T^{*} B$ of the Borel subgroup of $G$ [19].
} 

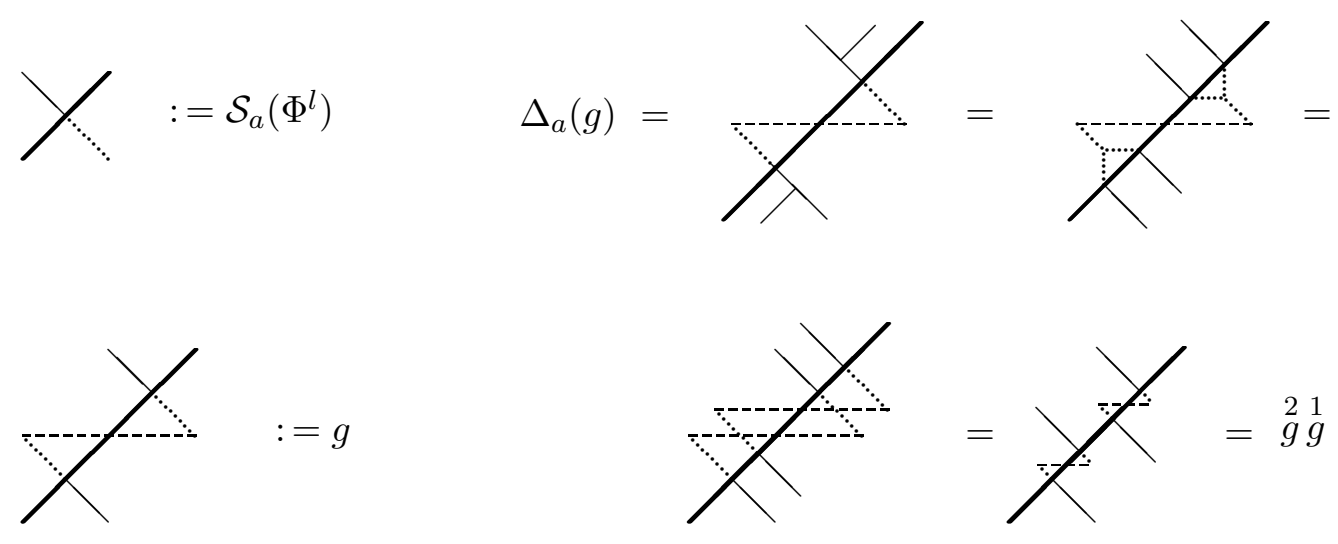

Figure 4: The definition of $g$ in terms of $\Phi^{r}$ and $\mathcal{S}_{a}\left(\Phi^{l}\right)$ is shown on the left side. The right side of the figure is a pictorial proof of the operator product expansion for $g$ (first eq. in (3.20)).

Before concluding this subsection we would like to compare our construction of $g$ with the one discussed in [2]. There, two decompositions of $g$ into triple products of elements, $g=u Q^{-1} v=u_{0} Q v_{0}$, have been provided. All operators which appear in these relations act on the diagonal subspace $\mathcal{H}$. The variables $v_{0}, u_{0}$ are chiral observables, i.e., $u_{0} \in \mathcal{G}_{a} \otimes \mathcal{J}^{l}, v_{0} \in \mathcal{G}_{a} \otimes \mathcal{J}^{r}$, and hence they commute with each other: $\stackrel{1}{u_{0}} \stackrel{2}{v_{0}}=\stackrel{2}{v_{0}} \stackrel{1}{u_{0}}$. Notice that components of $u_{0}, v_{0}$ leave the subspaces $V^{\bar{I}} \otimes V^{I} \subset \mathcal{H}$ invariant and hence their actions are, in principle, expressible through the chiral objects $M^{\alpha}$ (in particular, $u_{0}, v_{0}$ are not to be confused with our vertex operators). The exchange relations of $u_{0}$ (respectively $v_{0}$ ) can be controlled only after multiplication with $Q \in \mathcal{G}_{a} \otimes \operatorname{End}(\mathcal{H})$. In fact, the elements $u=u_{0} Q$ and $v=Q v_{0}$ possess the same exchange relations as our chiral vertex operators. On the other hand, they are certainly not chiral any more (because chiral vertex operators cannot act on $\mathcal{H}$ ). In particular, $u$ does not commute with $v$. One may think of $u$ (and similarly of $v$ ) as a left chiral vertex operator dressed with a right chiral factor which leaves the quadratic relations unchanged and, at the same time, produces an operator acting on $\mathcal{H}$. Our construction in terms of chiral vertex operators and the restriction from $\mathcal{M}$ to the diagonal subspace $\mathcal{H}$ is similar to [26, 35].

3.5 Toy model for $\mathcal{Z}_{q}$. It is instructive to realize the constructions of the toy model in the case of $\mathcal{G}=\mathcal{Z}_{q}$. Now we have two commuting copies, $h_{\alpha}, \alpha=r, l$, of the element $h$ (see Subsection 2.1) generating the chiral algebras $\mathcal{J}^{\alpha}$. We can also introduce Hermitian operators $\widehat{p}_{\alpha}$ such that $h_{\alpha}=q^{\widehat{p}_{\alpha}}$. To introduce the chiral monodromies $M^{r}$ and $M^{l}$ we use the expressions (2.13) for the elements $N$ and $\widetilde{N}$. Since $M^{r}$ and $M^{l}$ differ from them only by factors $v_{a}^{-1}$ and $v_{a}$, we get

$$
M^{r}=q^{\widehat{p}^{2} \otimes e+2 \widehat{p} \otimes \widehat{p}_{r}}, \quad M^{l}=q^{-\widehat{p}^{2} \otimes e-2 \widehat{p} \otimes \widehat{p}_{l}} .
$$

The reader is invited to verify the functorial properties (3.1), 3.6) for these objects (in fact, the check repeats the computations performed in Subsection 2.2). As was explained in Subsection 2.2, the element $S_{\iota}$ is trivial in the case of $\mathcal{Z}_{q}$, therefore the chiral monodromies are unitary. The components of $M^{\alpha}$ act on the model spaces $\mathcal{M}^{\alpha}$. 
Next we need to construct the diagonal subspace $\mathcal{H}=\oplus V^{\bar{K}} \otimes V^{K}$. It can be seen from the explicit formula for the characteristic projectors (2.3) that $\mathcal{S}_{a}\left(P^{s}\right)=P^{-s}$, i.e., the representation conjugate to $\tau^{s}$ is $\tau^{-s}$ (where $s$ is taken modulo $p, q^{p}=1$ ). Therefore, $\mathcal{H}=\oplus|-s\rangle \otimes|s\rangle$ is a $p$-dimensional subspace in $p^{2}$-dimensional space $\mathcal{M}^{l} \otimes \mathcal{M}^{r}$. Using the operators $\widehat{p}_{\alpha}$, we can characterize the subspace $\mathcal{H}$ as follows: $\widehat{p}_{r} \phi=-\widehat{p}_{l} \phi$ for all $\phi \in \mathcal{H}$.

Now we employ the construction for vertex operators which we provided in Subsection 2.5. Let $\widehat{Q}_{\alpha}, \alpha=r, l$ be unitary operators acting on $\mathcal{M}^{l} \otimes \mathcal{M}^{r}$ such that $\widehat{Q}_{r}\left|s^{\prime}\right\rangle \otimes\left|s^{\prime \prime}\right\rangle=$ $\left|s^{\prime}\right\rangle \otimes\left|s^{\prime \prime}+1\right\rangle$ and $\widehat{Q}_{l}\left|s^{\prime}\right\rangle \otimes\left|s^{\prime \prime}\right\rangle=\left|s^{\prime}+1\right\rangle \otimes\left|s^{\prime \prime}\right\rangle$. It is convenient to introduce also two operators $\widehat{\varsigma}_{\alpha}$ by $\widehat{Q}_{\alpha}=e^{\widehat{\varsigma}_{\alpha}}$. With these notations it is easy to verify that

$$
\Phi^{\alpha}=\sum_{s=0}^{p-1} P^{s} \otimes \widehat{Q}_{\alpha}^{s}=e^{\widehat{p} \otimes \widehat{\varsigma}_{\alpha}} \in \mathcal{G}_{a} \otimes \operatorname{End}\left(\mathcal{M}^{\alpha}\right), \quad \alpha=r, l
$$

are right- and left chiral operators obeying all the properties spelled out in Subsections 3.1 and 3.2, respectively. In particular, the $R$-matrix commutation relations in (3.3), (3.8) boil down to Weyl relations: $e^{e \otimes \widehat{p} \otimes \widehat{\varsigma}_{\alpha}} q^{ \pm 2 \widehat{p} \otimes e \otimes \widehat{p}_{r}}=q^{ \pm 2 \widehat{p} \otimes \widehat{p} \otimes e} q^{ \pm 2 \widehat{p} \otimes e \otimes \widehat{p}_{r}} e^{e \otimes \widehat{p} \otimes \widehat{\varsigma}_{\alpha}}$. Recall that the universal $R$-matrices in (3.3), (3.8) are regarded as elements in $\mathcal{G}_{a} \otimes \mathcal{G}_{a} \otimes \operatorname{End}(\mathcal{H})$

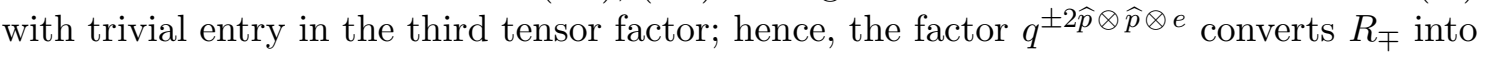
$R_{ \pm}$(cf. Subsection 2.2).

Now, applying (3.16), we get an explicit expression for $g$ :

$$
g=\sum_{s=0}^{p-1} P^{s} \otimes \widehat{Q}_{r}^{s} \widehat{Q}_{l}^{-s}=e^{\widehat{p} \otimes\left(\widehat{\varsigma}_{r}-\widehat{\varsigma}_{l}\right)} .
$$

This object manifestly maps the diagonal subspace into itself and hence we may regard $g$ as an element in $\mathcal{G}_{a} \otimes \operatorname{End}(\mathcal{H})$. The operator product expansion (3.20) is obvious (see the analogous computation for $N_{ \pm}$in Subsection 2.2). Moreover, the first equation in (3.22) is again of Weyl-type (notice that here the factors $v_{a}^{\mp 1}$ of $M^{\alpha}$ are essential):

$$
\begin{gathered}
M^{l} g=q^{-\widehat{p}^{2} \otimes e} q^{-2 \widehat{p} \otimes \widehat{p}_{l}} e^{\widehat{p} \otimes\left(\widehat{\varsigma}_{r}-\widehat{\varsigma}_{l}\right)}=q^{-\widehat{p}^{2} \otimes e} e^{\widehat{p} \otimes\left(\widehat{\varsigma}_{r}-\widehat{\varsigma}_{l}\right)} q^{-2 \widehat{p} \otimes \widehat{p}_{l}} q^{2 \widehat{p}^{2} \otimes e}= \\
=e^{\widehat{p} \otimes\left(\widehat{\varsigma}_{r}-\widehat{\varsigma}_{l}\right)} q^{\widehat{p}^{2} \otimes e} q^{-2 \widehat{p} \otimes \widehat{p}_{l}}=e^{\widehat{p} \otimes\left(\widehat{\varsigma}_{r}-\widehat{\varsigma}_{l}\right)} q^{\widehat{p}^{2} \otimes e} q^{2 \widehat{p} \otimes \widehat{p}_{r}}=g M^{r} .
\end{gathered}
$$

In the last line we used the constraint $\widehat{p}_{r}=-\widehat{p}_{l}$ valid on the diagonal subspace. To conclude, we notice that in the $\mathcal{Z}_{q}$ case the vertex operators and the field $g$ are unitary.

\section{REVIEW ON LATTICE CURRENT ALGEBRAS}

In the previous section we have considered the toy model for the WZNW theory which certainly did not go much beyond the theory of vertex operators for quasi-triangular modular Hopf algebras (except that we had two commuting copies of this theory). Vertex operators for the infinite dimensional current algebras of the WZNW-model depend in addition on a spatial coordinate $x$. This brings new locality features into the theory. Our aim is to describe them for a lattice regularization of the WZNW-model developed in [4, 5, 26, 33, 6], where the spatial coordinate assumes the discrete values, $x=2 \pi n / N$, $n=0, . ., N-1$. We begin this discussion with a brief review on lattice current algebras $\mathcal{K}_{N}$. Our notations are close to those adopted in $[6]$. 
4.1 Definition of lattice current algebras. Let us consider a one-dimensional periodic lattice which consists of $N$ vertices. It is convenient to enumerate the vertices from 0 to $N-1$ and the corresponding edges from 1 to $\mathrm{N}$ as shown below.

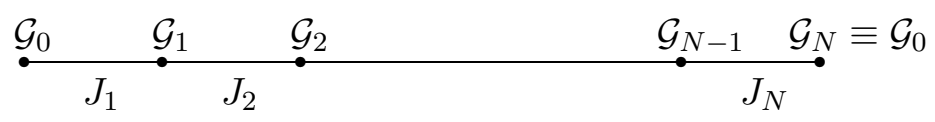

Figure 5: $N$-vertex periodic lattice. Each site is equipped with a copy of the symmetry algebra $\mathcal{G}$. The discrete currents $J_{n}$ are assigned to edges.

According to the ideology of [6], the definition of the algebra $\mathcal{K}_{N}$ involves two kinds of objects - those associated with the sites and those associated with the edges. The $n^{\text {th }}$ site of the lattice is equipped with a copy $\mathcal{G}_{n}$ of the algebra $\mathcal{G}$ and copies for different sites commute. In other words, $\mathcal{K}_{N}$ contains $\mathcal{G}_{n}$ and the whole tensor product $\mathcal{G}^{\otimes N}$ as subalgebras. The canonical isomorphism of $\mathcal{G}$ and $\mathcal{G}_{n} \subset \mathcal{G}^{\otimes N}$ furnishes the embeddings $\iota_{n}: \mathcal{G} \mapsto \mathcal{G}^{\otimes N}$ for $n=0, . ., N-1$ :

$$
\iota_{n}(\xi)=e \otimes \ldots \otimes \xi \otimes \ldots \otimes e \in \mathcal{G}^{\otimes N} \quad \text { for all } \xi \in \mathcal{G},
$$

where the only nontrivial entry of the tensor product on r.h.s. appears in the $n^{\text {th }}$ position. The definition of $\mathcal{K}_{N}$ also involves generators $J_{n}^{r}, n=1, . ., N$, (the right currents) which are discrete analogues of the continuum holonomies along the edges (cf. Introduction).

Definition 2 [6] The lattice current algebra $\mathcal{K}_{N}$ is generated by components of invertible elements $J_{n}^{r} \in \mathcal{G}_{a} \otimes \mathcal{K}_{N}, n=1, . ., N$ along with elements in $\mathcal{G}^{\otimes N}$. These generators are subject to the following relations :

$$
\begin{aligned}
& \stackrel{2}{J}_{n}^{r} \stackrel{1}{J}_{n}^{r}=R_{-} \Delta_{a}\left(J^{r}\right), \quad\left(J_{n}^{r}\right)^{*}=S_{n}^{-1}\left(J_{n}^{r}\right)^{-1} S_{n-1} \\
& \stackrel{1}{J_{n+1}^{r}} \stackrel{\stackrel{2}{J}}{n}=\stackrel{2}{J_{n}^{r}} R_{+} \stackrel{1}{J_{n+1}^{r}}, \quad \stackrel{1}{J_{n}^{r}} \stackrel{2}{J}_{m}^{r}=\stackrel{2}{J}_{m}^{r} \stackrel{1}{J_{n}^{r}} \quad \text { for } n \neq m, m \pm 1(\bmod N), \\
& \iota_{n}(\xi) J_{n}^{r}=J_{n}^{r} \Delta_{n}^{\prime}(\xi), \quad \Delta_{n-1}^{\prime}(\xi) J_{n}^{r}=J_{n}^{r} \iota_{n-1}(\xi) \quad \text { for all } \xi \in \mathcal{G}, \\
& \iota_{m}(\xi) J_{n}^{r}=J_{n}^{r} \iota_{m}(\xi) \quad \text { for all } \xi \in \mathcal{G}, m \neq n, n-1(\bmod N) \text {. }
\end{aligned}
$$

Here $R_{ \pm}$denote the elements $R_{ \pm} \otimes \mathrm{e} \in \mathcal{G}_{a} \otimes \mathcal{G}_{a} \otimes \mathcal{K}_{N}, S_{n}=\left(i d \otimes \iota_{n}\right)(S) \in \mathcal{G}_{a} \otimes \mathcal{G}_{n} \subset \mathcal{G}_{a} \otimes \mathcal{K}_{N}$ with $S$ defined as in 2.10), and $\Delta_{n}^{\prime}(\xi)=\left(i d \otimes \iota_{n}\right)\left(\Delta^{\prime}(\xi)\right)$ where $\Delta^{\prime}(\xi)=P \Delta(\xi) P$ as usual. Invertibility of $J_{n}^{r}$ means that there exists an element $\left(J_{n}^{r}\right)^{-1} \in \mathcal{G}_{a} \otimes \mathcal{K}_{N}$ such that $J_{n}^{r}\left(J_{n}^{r}\right)^{-1}=e \otimes \mathrm{e}=\left(J_{n}^{r}\right)^{-1} J_{n}^{r}$.

The lattice current algebra $\mathcal{K}_{N}$ contains a subalgebra $\mathcal{J}_{N}^{r}$ generated by components of the currents $J_{n}^{r}$ only. They are subject to relations (4.1)-(4.2). The full lattice current algebra $\mathcal{K}_{N}$ can be regarded as a semi-direct product $\mathcal{G}^{\otimes N} \ltimes \mathcal{J}_{N}^{r}$, where the action of $\mathcal{G}^{\otimes N}$ on $\mathcal{J}_{N}^{r}$ is given by the covariance relations (4.3).

Taking into account the quasi-triangularity of $R_{ \pm}$, we obtain the following consequence of (4.1)

$$
R_{ \pm} \stackrel{1}{J_{n}^{r}} \quad \stackrel{2}{J_{n}^{r}} R_{\mp}=\stackrel{2}{J_{n}^{r}} \stackrel{1}{J_{n}^{r}}
$$

These $R$-matrix relations for the description of the lattice Kac-Moody algebras have been introduced first in 泪. Following our discussion in Subsection 2.2, one can introduce the objects $N_{n, \pm}=\left(i d \otimes \iota_{n}\right)\left(R_{ \pm}\right) \in \mathcal{G}_{a} \otimes \mathcal{G}_{n} \subset \mathcal{G}_{a} \otimes \mathcal{K}_{N}$, which obey the standard relations (2.5). They are used to rewrite the relations (4.3) in the following $R$-matrix form

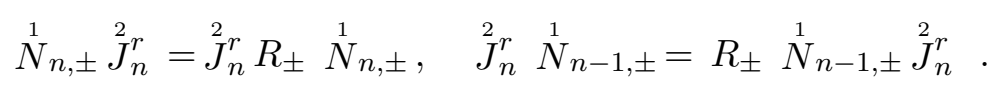


4.2 Left currents. The continuum WZNW model possesses two chiral subalgebras, that is, along with the (right) current $j^{r}(x)$ it involves the left current $j^{l}(x)$ such that left and right currents commute. A nice feature of the lattice current algebra $\mathcal{K}_{N}$ is that it already contains the second chirality in an encoded form. Indeed, one may introduce the following new variables $J_{n}^{l} \in \mathcal{G}_{a} \otimes \mathcal{K}_{N}$ :

$$
J_{n}^{l}:=v_{a}^{2} N_{n-1,+}^{-1} J_{n}^{r} N_{n,-} .
$$

In the notations of Definition 2 they obey (see [6] for details)

$$
\begin{aligned}
& \stackrel{1}{J}_{n}^{l} \quad \stackrel{2}{J}_{n}^{l}=R_{+} \Delta_{a}\left(J_{n}^{l}\right) \quad, \quad\left(J_{n}^{l}\right)^{*}=S_{n}\left(J_{n}^{l}\right)^{-1} S_{n-1}^{-1},
\end{aligned}
$$

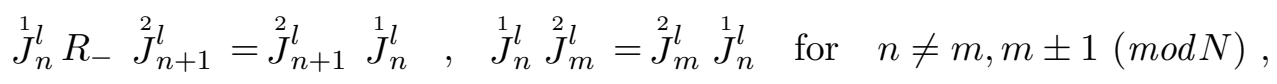

$$
\begin{aligned}
& \stackrel{1}{J}_{n}^{l} \stackrel{2}{J}_{m}^{r}=\stackrel{2}{J}_{m}^{r} \stackrel{1}{J_{n}^{l}} \quad \text { for all } m, n, \\
& \iota_{n}(\xi) J_{n}^{l}=J_{n}^{l} \Delta_{n}(\xi) \quad, \quad \Delta_{n-1}(\xi) J_{n}^{l}=J_{n}^{l} \iota_{n-1}(\xi) \text { for all } \xi \in \mathcal{G} \text {, } \\
& \iota_{m}(\xi) J_{n}^{l}=J_{n}^{l} \iota_{m}(\xi) \quad \text { for all } \xi \in \mathcal{G}, m \neq n, n-1(\bmod N) \text {. }
\end{aligned}
$$

Due to these properties, the objects $J_{n}^{l}$ may be interpreted as left counterparts of the right currents $J_{n}^{r}$. Notice that there is a manifest symmetry between the defining relations for the right currents and the properties of left currents. It underlines the fact that left and right chiralities in the WZNW model appear on an equal footing. In fact, (4.7)-(4.10) could be regarded as an alternative definition of the lattice current algebra $\mathcal{K}_{N}$.

It also follows that $\mathcal{J}_{N}^{l}$ and $\mathcal{J}_{N}^{r}$, i.e., the algebras generated by components of $J_{n}^{l}$ and $J_{n}^{r}$, respectively, are commuting subalgebras in $\mathcal{K}_{N}$ and $\mathcal{J}_{N}^{r}$ is isomorphic to $\left(\mathcal{J}_{N}^{l}\right)_{\text {op }}$. Here the subscript op means opposite multiplication as before.

4.3 Holonomies and monodromies. The currents $J_{n}^{\alpha}, \alpha=r, l$ were defined as discrete analogues of holonomies along the $n^{\text {th }}$ edge. Similarly, one may introduce the holonomies along the link connecting the $0^{\text {th }}$ and the $n^{\text {th }}$ sites :

$$
U_{n}^{\alpha}:=J_{1}^{\alpha} \ldots J_{n}^{\alpha}, \quad n=1, \ldots, N-1
$$

As one might expect, the properties of such holonomies are similar to those of chiral currents. Namely, it is easy to verify that

$$
\begin{aligned}
& \stackrel{2}{U^{r}} \stackrel{1}{{ }^{\prime}}{ }_{n}^{r}=R_{-} \Delta_{a}\left(U_{n}^{r}\right) \quad, \quad \stackrel{1}{U}_{n}^{l} \stackrel{2}{U_{n}^{l}}=R_{+} \Delta_{a}\left(U_{n}^{l}\right), \\
& \left(U_{n}^{r}\right)^{*}=S_{n}^{-1}\left(U_{n}^{r}\right)^{-1} S_{0} \quad, \quad\left(U_{n}^{l}\right)^{*}=S_{n}\left(U_{n}^{l}\right)^{-1} S_{0}^{-1},
\end{aligned}
$$

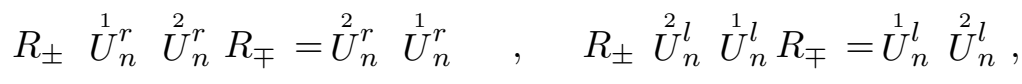

$$
\begin{aligned}
& \Delta_{0}^{\prime}(\xi) U_{n}^{r}=U_{n}^{r} \iota_{0}(\xi) \quad, \quad \iota_{n}(\xi) U_{n}^{r}=U_{n}^{r} \Delta_{n}^{\prime}(\xi) \quad \text { for all } \xi \in \mathcal{G}, \\
& \Delta_{0}(\xi) U_{n}^{l}=U_{n}^{l} \iota_{0}(\xi) \quad, \quad \iota_{n}(\xi) U_{n}^{l}=U_{n}^{l} \Delta_{n}(\xi) \text { for all } \xi \in \mathcal{G}
\end{aligned}
$$

\footnotetext{
${ }^{12}$ Let us mention here some subtle point in the definition of the lattice current algebra. Notice that relations (4.4) would not change if we replaced $R_{-}$by $R_{+}$in the definition (4.1). However, this ambiguity disappears if we demand that $U_{n}^{r}$ and $J_{n}^{r}$ have the same functoriality relation (compare (4.1) and first eq. in (4.12). A similar subtlety appears once more in the construction of the left currents. Indeed, we could replace factor $v_{a}^{2}$ by $v_{a}$ in the definition (4.6); then we would obtain the relation (4.7) with $R_{-}$instead of $R_{+}$. But in this case functorial properties of $J_{n}^{l}$ and $U_{n}^{l}$ would be different.
} 
and $U_{n}^{\alpha}$ commute with $\iota_{m}(\xi)$ for all $m \neq 0, n$. However, there is an important difference between currents and holonomies: since the latter are localized on the chain of edges that runs from the $0^{t h}$ vertex to the $n^{\text {th }}$, the localization domains of all holonomies overlap. This is reflected in their mutual exchange relations for $1 \leq n<m \leq N-1$ :

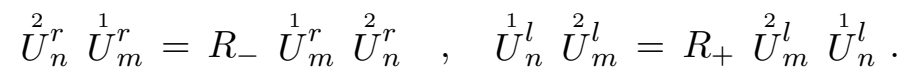

As we have argued in the introduction, holonomies of chiral fields along the whole circle (i.e., the chiral monodromies) are of particular interest. In the continuum case they are given by $m^{\alpha}=\mathcal{P} \exp \left\{\oint j^{\alpha}(x) d x\right\}$. Monodromies for the quantum lattice theory are defined by a natural discrete analogue of this formula,

$$
M^{\alpha}=J_{1}^{\alpha} J_{2}^{\alpha} \ldots J_{N}^{\alpha} .
$$

Simple calculations allow to derive the following properties of the monodromies $M^{\alpha}$ :

$$
\begin{aligned}
& \stackrel{2}{M}^{r} R_{+} \stackrel{1}{M}^{r}=R_{-} \Delta_{a}\left(M^{r}\right) \quad, \quad M^{l} R_{-} \stackrel{2}{M}^{l}=R_{+} \Delta_{a}\left(M^{l}\right), \\
& \left(M^{r}\right)^{*}=S_{0}^{-1}\left(M^{r}\right)^{-1} S_{0} \quad, \quad\left(M^{l}\right)^{*}=S_{0}\left(M^{l}\right)^{-1} S_{0}^{-1}, \\
& R_{+} \stackrel{1}{U_{n}^{r}} \stackrel{2}{M}^{r}=\stackrel{2}{M}^{r} R_{+} \stackrel{1}{U_{n}^{r}} \quad, \quad R_{-} \stackrel{2}{U}_{n}^{l} \stackrel{1}{M}^{l}=\stackrel{1}{M}^{l} R_{-} \stackrel{2}{U}_{n}^{l}, \\
& \Delta_{0}^{\prime}(\xi) M^{r}=M^{r} \Delta_{0}^{\prime}(\xi) \quad, \quad R_{ \pm} \stackrel{1}{N}{ }_{0, \pm} \stackrel{2}{M}^{r}=\stackrel{2}{M}^{r} R_{ \pm} \stackrel{1}{N}{ }_{0, \pm}, \\
& \Delta_{0}(\xi) M^{l}=M^{l} \Delta_{0}(\xi) \quad, \quad \stackrel{1}{N}{ }_{0, \pm} R_{ \pm} \stackrel{2}{M}^{l}=\stackrel{2}{M}^{l} \stackrel{1}{N}{ }_{0, \pm} R_{ \pm}
\end{aligned}
$$

for all $\xi \in \mathcal{G}$ and $M^{\alpha}$ commute with $\iota_{m}(\xi)$ for all $m \neq 0$.

Now we see that the relations (3.1) and (3.6) which we postulated in the toy model construction indeed describe properties of the chiral monodromies. Our next aim is to extend the toy model to the full lattice theory. Recall that the structure data of the toy model were built from elements in the center $\mathcal{C}^{\alpha}$ of the algebra $\mathcal{J}^{\alpha}$ spanned by components of $M^{\alpha}$. Elements in these algebras $\mathcal{C}^{\alpha}$ are still central in the full lattice theory. In fact, it follows from (3.2), (3.7) that the algebras $\mathcal{C}^{\alpha}$ are spanned by the elements $c_{\alpha}^{I}=\operatorname{tr}_{q}^{I} \tau^{I}\left(M^{\alpha}\right)$ where $\tau^{I}$ runs through irreducible representations of $\mathcal{G}$ and $\tau^{I}\left(M^{\alpha}\right)=\left(\tau^{I} \otimes i d\right)\left(M^{\alpha}\right)$ [7]. Equipped with this explicit description of $\mathcal{C}^{\alpha}$ one concludes from eqs. (4.21)-(4.23) that the elements $c_{\alpha}^{I}$ commute with $U_{n}^{\alpha}, N_{n, \pm}$ for all $n$ and hence they are central elements in $\mathcal{K}_{N}$. Actually, the following stronger statement holds $[6]$ : the elements $c_{\alpha}^{I} \in \mathcal{K}_{N}$ with $I$ running through the classes of irreducible representations of $\mathcal{G}$ generate the full center of the lattice current algebra $\mathcal{K}_{N}$. This explains why the structure data for vertex operators on the lattice will be built from the commuting subalgebras $\mathcal{C}^{\alpha}$ exactly as in the toy model.

4.4 Current algebra for $\mathcal{Z}_{q}$. Let us consider the current algebra in the case of $\mathcal{Z}_{q}$. The algebras $\mathcal{G}_{n}$ assigned to the sites are generated by the elements $h_{n}=\iota_{n}(h)$. As usual, we can introduce $\widehat{p}_{n}$ such that $h_{n}=q^{\widehat{p}_{n}}$. For $N_{n, \pm} \in \mathcal{G}_{a} \otimes \mathcal{G}_{n}$ we have $N_{n, \pm}=$ $\sum_{s} P^{s} \otimes h_{n}^{ \pm s}=q^{ \pm \hat{p} \otimes \widehat{p}_{n}}$ (cf. Subsection 2.2). Next we build the chiral currents

$$
J_{n}^{r}=\sum_{s=0}^{p-1} q^{\frac{1}{2} s^{2}} P^{s} \otimes\left(\widehat{W}_{n}^{r}\right)^{s} \quad, \quad J_{n}^{l}=\sum_{s=0}^{p-1} q^{-\frac{1}{2} s^{2}} P^{s} \otimes\left(\widehat{W}_{n}^{l}\right)^{s}
$$

from a family of unitary elements $\widehat{W}_{n}^{r}$ and $\widehat{W}_{n}^{l}:=h_{n-1}^{-1} \widehat{W}_{n}^{r} h_{n}^{-1}$ which obey the following Weyl-type relations

$$
h_{n} \widehat{W}_{n}^{\alpha}=q \widehat{W}_{n}^{\alpha} h_{n}, \quad h_{n-1} \widehat{W}_{n}^{\alpha}=q^{-1} \widehat{W}_{n}^{\alpha} h_{n-1},
$$




$$
\begin{aligned}
h_{m} \widehat{W}_{n}^{\alpha}=\widehat{W}_{n}^{\alpha} h_{m} & \text { for } m \neq n, n-1, \\
\widehat{W}_{n+1}^{r} \widehat{W}_{n}^{r}=q \widehat{W}_{n}^{r} \widehat{W}_{n+1}^{r}, & \widehat{W}_{n+1}^{l} \widehat{W}_{n}^{l}=q^{-1} \widehat{W}_{n}^{l} \widehat{W}_{n+1}^{l}, \\
\widehat{W}_{n}^{\alpha} \widehat{W}_{m}^{\alpha}=\widehat{W}_{m}^{\alpha} \widehat{W}_{n}^{\alpha} & \text { for } m \neq n \pm 1 .
\end{aligned}
$$

Since the element $\left(\widehat{W}_{n}^{\alpha}\right)^{p}$ is obviously central for this algebra, we additionally impose the condition: $\left(\widehat{W}_{n}^{\alpha}\right)^{p}=e$ for all $n$ (which is, in fact, a choice of a normalization).

The algebra generated by $\widehat{W}_{n}^{\alpha}$ is known as lattice $U(1)$-current algebra [31, 28]. The relation we have used to obtain the elements $\widehat{W}_{n}^{l}$ from the $h_{m}$ and $\widehat{W}_{n}^{r}$ is a special case of formula (4.6) and it implies that $\widehat{W}_{n}^{l} \widehat{W}_{m}^{r}=\widehat{W}_{m}^{r} \widehat{W}_{n}^{l}$ for all pairs $n, m$.

The functorial properties (4.1) and (4.7) of currents (4.24) can be checked in the same way as we did this for the elements $N_{ \pm}$in Subsection 2.2. The exchange relations (4.2), (4.5) and (4.8) are again reduced to Weyl relations. Since $S_{n}=e \otimes e$, the chiral currents are unitary, $\left(J_{n}^{\alpha}\right)^{*}=\left(J_{n}^{\alpha}\right)^{-1}$, which is in agreement with the unitarity of $\widehat{W}_{n}^{\alpha}$.

To proceed, we introduce anti-Hermitian operators $\widehat{\varpi}_{n}^{\alpha}$ such that $\widehat{W}_{n}^{\alpha}=e^{\widehat{\varpi}_{n}^{\alpha}} \cdot[3$ In these notations the commutation relations given above acquire the form:

$$
\begin{array}{ll}
{\left[\widehat{\varpi}_{m}^{r}, \widehat{\varpi}_{n}^{r}\right]=\ln q\left(\delta_{m, n+1}-\delta_{m, n-1}\right)} & ,\left[\widehat{\varpi}_{m}^{l}, \widehat{\varpi}_{n}^{l}\right]=\ln q\left(\delta_{m, n-1}-\delta_{m, n+1}\right), \\
{\left[\widehat{p}_{m}, \widehat{\varpi}_{n}^{\alpha}\right]=\delta_{m, n}-\delta_{m, n-1},} & {\left[\widehat{\varpi}_{m}^{l}, \widehat{\varpi}_{n}^{r}\right]=0 .}
\end{array}
$$

The chiral currents now can be rewritten in the following form:

$$
J_{n}^{r}=\kappa_{a}^{-1} e^{\widehat{p} \otimes \widehat{\varpi}_{n}^{r}}=e^{\left(\frac{1}{2} \ln q\right) \widehat{p}^{2} \otimes e+\widehat{p} \otimes \widehat{\varpi}_{n}^{r}}, \quad J_{n}^{l}=\kappa_{a} e^{\widehat{\hat{p} \otimes \widehat{\varpi}_{n}^{l}}}=e^{-\left(\frac{1}{2} \ln q\right) \widehat{p}^{2} \otimes e+\widehat{p} \otimes \widehat{\varpi}_{n}^{l}} .
$$

Next we can construct the chiral holonomies. For this purpose the variables $\widehat{\varpi}_{n}^{\alpha}$ are more convenient. Indeed, applying the special case of the Campbell-Hausdorff formula, $e^{a} e^{b}=e^{a+b} e^{\frac{1}{2}[a, b]}$ valid if $[a,[a, b]]=[b,[a, b]]=0$, we easily obtain:

$$
U_{n}^{r}=q^{\frac{1}{2} \widehat{p}^{2} \otimes e} e^{\widehat{p} \otimes \sum_{k=1}^{n} \widehat{\varpi}_{k}^{r}}, \quad U_{n}^{l}=q^{-\frac{1}{2} \widehat{p}^{2} \otimes e} e^{\widehat{p} \otimes \sum_{k=1}^{n} \widehat{\varpi}_{k}^{l}}
$$

It is obvious now why relations (4.12)-(4.16) for the holonomies copy those for the currents. The exchange relations (4.17) are again reduced to Weyl-type relations.

Proceeding in the same way, we can construct the chiral monodromies as $M^{\alpha}=$ $U_{N-1}^{\alpha} J_{N}^{\alpha}$, which needs again an application of the Campbell-Hausdorff formula. The result reads

$$
M^{r}=q^{\widehat{p}^{2} \otimes e} e^{\widehat{p} \otimes \sum_{k=1}^{N} \widehat{\varpi}_{k}^{r}}, \quad M^{l}=q^{-\widehat{p}^{2} \otimes e} e^{\widehat{p} \otimes \sum_{k=1}^{N} \widehat{\varpi}_{k}^{l}} .
$$

Bearing in mind that for $\mathcal{Z}_{q}$ the quantum trace coincides with the standard one (see Subsection 2.1), we conclude from (4.29) that the algebras $\mathcal{C}^{\alpha}$ are generated by exponentials of the elements $\widehat{p}_{\alpha}=\sum_{k=1}^{N} \widehat{\varpi}_{k}^{\alpha}$. Indeed, using the commutation relations given above, it is easy to verify that these operators commute with all elements of the currents algebra. Performing a formal replacement $\sum_{k=1}^{N} \widehat{\varpi}_{k}^{\alpha} \rightarrow \pm(2 \ln q) \widehat{p}_{\alpha}$ in $(4.29)$ (the sign depends on the chirality), we recover the formulae (3.23) of the toy model.

\section{VERTEX OPERATORS ON A LATTICE}

\footnotetext{
${ }^{13}$ Strictly speaking, the algebra generated by $\widehat{\varpi}_{n}^{\alpha}$ and $\widehat{p}_{n}$ is larger than one generated by $\widehat{W}_{n}^{\alpha}$ and $h_{n}$ (see, e.g., 28]). The latter is called compactified form of the former.
} 
5.1 Definition of $\mathcal{W}_{N}$. In Section 3 we have considered algebras $\mathcal{J}^{\alpha}$ and $\mathcal{V}^{\alpha}, \alpha=r, l$ generated by components of the chiral monodromies $M^{\alpha}$ and the chiral vertex operators $\Phi^{\alpha}$, respectively. Both chiralities together were used to generate the algebra $\mathcal{W}=\mathcal{V}^{l} \otimes$ $\mathcal{V}^{r}$ of our toy model. Below we shall define an algebra $\mathcal{W}_{N}$ of vertex operators on a lattice. For this purpose, we shall replace the algebras $\mathcal{J}^{\alpha}$ in the definition of $\mathcal{W}$ by their lattice counterparts $\mathcal{J}_{N}^{\alpha}$. So we assume that we are given the lattice current algebra $\mathcal{K}_{N}$ with center $\mathcal{C}^{l} \otimes \mathcal{C}^{r}$ (recall that $\mathcal{C}^{\alpha} \cong \mathcal{C} \cong$ center of $\mathcal{G}$ ) and two sets of structure data $F_{\alpha}, \sigma_{\alpha}, \mathcal{R}_{ \pm}^{\alpha}, D_{\alpha}, \alpha=l, r$, which obey the standard relations. The last tensor components of the structure data are regarded as elements in the center of the lattice current algebra $\mathcal{K}_{N}$, i.e., we have $F_{\alpha} \in \mathcal{G}_{a} \otimes \mathcal{G}_{a} \otimes \mathcal{C}^{\alpha} \subset \mathcal{G}_{a} \otimes \mathcal{G}_{a} \otimes \mathcal{K}_{N}$ etc.

Definition 3 (Algebra of vertex operators on a lattice) The algebra $\mathcal{W}_{N}$ is generated by elements in $\mathcal{K}_{N}$ and components of the vertex operators $\Phi_{0}^{\alpha} \in \mathcal{G}_{a} \otimes \mathcal{W}_{N}$. Generators $N_{n}, J_{n}^{\alpha} \in \mathcal{G}_{a} \otimes \mathcal{K}_{N}$ obey the defining relations (4.1), (4.5) and (4.5) for lattice current algebras. The elements $\Phi_{0}^{\alpha} \in \mathcal{G}_{a} \otimes \mathcal{V}^{\alpha} \subset \mathcal{G}_{a} \otimes \mathcal{W}_{N}, \alpha=r, l$ are subject to the following conditions:

1. They satisfy operator product expansions and exchange relations with elements in the center $\mathcal{C}^{l} \otimes \mathcal{C}^{r} \subset \mathcal{K}_{N}$ given by

$$
\begin{aligned}
& \stackrel{2}{\Phi}_{0}^{r} \stackrel{\stackrel{1}{\Phi}}{{ }_{0}^{r}}=F_{r} \Delta_{a}\left(\Phi_{0}^{r}\right) \quad, \quad \Phi_{0}^{r} \mathrm{f}_{r}=\sigma_{r}\left(\mathrm{f}_{r}\right) \Phi_{0}^{r}, \\
& \stackrel{1}{\Phi_{0}^{l}} \stackrel{2}{\Phi_{0}^{l}}=F_{l} \Delta_{a}\left(\Phi_{0}^{l}\right) \quad, \quad \Phi_{0}^{l} \mathrm{f}_{l}=\sigma_{l}\left(\mathrm{f}_{l}\right) \Phi_{0}^{l} .
\end{aligned}
$$

Here $F_{\alpha} \in \mathcal{G}_{a} \otimes \mathcal{G}_{a} \otimes \mathcal{C}^{\alpha} ; \sigma_{\alpha}$ are homomorphisms from $\mathcal{C}^{\alpha}$ to $\mathcal{G}_{a} \otimes \mathcal{C}^{\alpha}$ and $\mathrm{f}_{\alpha} \in \mathcal{C}^{\alpha}$. Moreover, $\Phi_{0}^{\alpha}$ are invertible and vertex operators of different chirality commute:

$$
\left(\Phi_{0}^{\alpha}\right)^{-1} \Phi_{0}^{\alpha}=e \otimes \mathrm{e}=\Phi_{0}^{\alpha}\left(\Phi_{0}^{\alpha}\right)^{-1}, \alpha=l, r, \quad \stackrel{1}{\Phi_{0}^{r}} \stackrel{2}{\Phi_{0}^{l}}=\stackrel{2}{\Phi_{0}^{l}} \stackrel{1}{\Phi_{0}^{r}} .
$$

2. $\Phi_{0}^{r}$ and $\Phi_{0}^{l}$ are chiral vertex operators for the algebra $\mathcal{K}_{N}$ in the sense that the following exchange relations with $J_{n}^{r}, N_{n, \pm} \in \mathcal{G}_{a} \otimes \mathcal{K}_{N}$ hold:

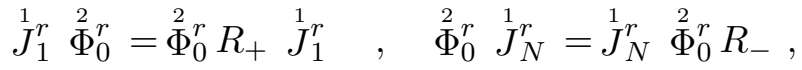

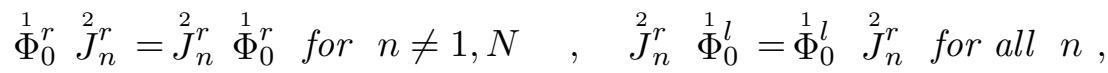

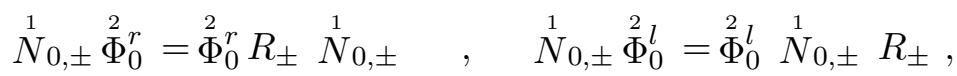

and components of $N_{m, \pm}$ commute with components of the vertex operators for $m \neq 0$.

3. The *-operation on $\mathcal{K}_{N}$ can be extended to $\mathcal{W}_{N}$ by the following prescription

$$
\left(\Phi_{0}^{r}\right)^{*}=\left(S_{0}\right)^{-1}\left(\Phi_{0}^{r}\right)^{-1} \quad, \quad\left(\Phi_{0}^{l}\right)^{*}=S_{0}\left(\Phi_{0}^{l}\right)^{-1},
$$

where $S_{0}=\left(i d \otimes \iota_{0}\right)(S) \in \mathcal{G}_{a} \otimes \mathcal{G}_{0} \subset \mathcal{G}_{a} \otimes \mathcal{K}_{N}$ with $S \in \mathcal{G}_{a} \otimes \mathcal{G}$ being constructed by formula (2.10).

Further relations involving left currents $J_{n}^{l}$ and the monodromies $M^{\alpha}$ follow and will be spelled out below. 
Let us underline once more that the structure data for $\Phi_{0}^{\alpha}$ are constructed from elements in the center $\mathcal{C}^{l} \otimes \mathcal{C}^{r}$ of $\mathcal{K}_{N}$. This is possible because both algebras $\mathcal{C}^{\alpha}$ are isomorphic to the center of $\mathcal{G}$ [6] (see our short discussion at the end of Subsection 4.3).

Next we would like to supplement our definition of $\mathcal{W}_{N}$ by a list of consequences which follow from the stated relations. They concern exchange relations of chiral vertex operators with left currents $J_{n}^{l}$ and elements $\xi \in \mathcal{G}_{n} \subset \mathcal{K}_{N}$,

$$
\begin{aligned}
& \stackrel{2}{J}_{1}^{l} \stackrel{1}{\Phi_{0}^{l}}=\stackrel{1}{\Phi}{ }_{0}^{l} R_{-} \stackrel{2}{J_{1}^{l}} \quad, \quad \stackrel{1}{\Phi}{ }_{0}^{l} \stackrel{2}{J_{N}^{l}}=\stackrel{2}{J_{N}^{l}} \stackrel{1}{\Phi_{0}^{l}} R_{+},
\end{aligned}
$$

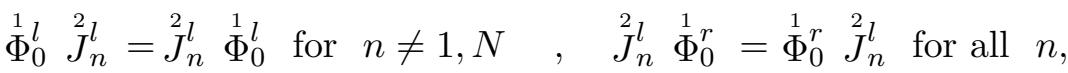

$$
\begin{aligned}
& \iota_{0}(\xi) \Phi_{0}^{r}=\Phi_{0}^{r} \Delta_{0}^{\prime}(\xi) \quad, \quad \iota_{0}(\xi) \Phi_{0}^{l}=\Phi_{0}^{l} \Delta_{0}(\xi), \\
& \iota_{m}(\xi) \Phi_{0}^{r}=\Phi_{0}^{r} \iota_{m}(\xi) \quad, \quad \iota_{m}(\xi) \Phi_{0}^{l}=\Phi_{0}^{l} \iota_{m}(\xi) \quad \text { for } m \neq 0,
\end{aligned}
$$

for all $\xi \in \mathcal{G}$ and we used the same notations as in Subsection 4.1. The first set of relations, i.e. eqs. (5.8),(5.9), are obtained with the help of eq. (4.6). From our earlier discussion it is clear that the relations (5.10) are equivalent to eqs. (5.6). All exchange relations of the chiral vertex operators with elements in $\mathcal{K}_{N}$ are local in the sense that objects assigned to sites $n \neq 0$ or links $m \neq 1, N$ commute with $\Phi_{0}^{\alpha}$. This means that we can think of $\Phi_{0}^{\alpha}$ as being assigned to the vertex $n=0$ and hence explains the subscripts 0 . The precise form of the nontrivial exchange relations involving $\Phi_{0}^{\alpha}$ may be understood in terms of co-actions of $\mathcal{G}$ on $\mathcal{K}_{N}$ (see remarks in the introduction and [4, 6]).

Now let us compare the definition of algebras $\mathcal{W}_{N}$ of vertex operators on the lattice with our toy model. To this end we derive exchange relations between the chiral vertex operators $\Phi_{0}^{\alpha}$ and the chiral monodromies $M^{\alpha}$ (see eqs. (4.18)),

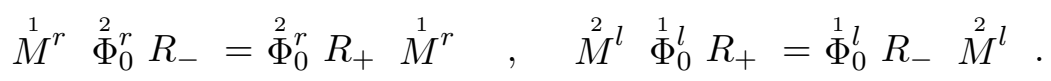

The answer is to be compared with the relations (3.3), (3.8) in the toy model and shows that the objects $\left(\Phi_{0}^{\alpha}, M^{\alpha}, N_{0}\right)$ of the algebra $\mathcal{W}_{N}$ obey the same exchange algebra as $\left(\Phi^{\alpha}, M^{\alpha}, N\right)$ in the toy model. Thus, the toy model may not only be considered as a special case of a lattice theory with $N=1$ but also it is embedded as a subalgebra in all lattice algebras $\mathcal{W}_{N}$ for arbitrary $N$. We can use this insight to rewrite some of the relations we discussed for the toy model in terms of the corresponding lattice objects. In particular, one has

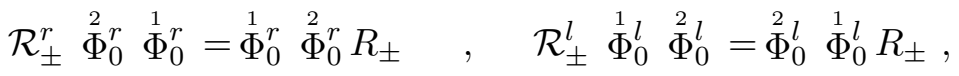

$$
\begin{aligned}
& v_{a}^{-1}\left(\Phi_{0}^{r}\right)^{-1} D_{r} \Phi_{0}^{r}=M^{r} \quad, \quad v_{a}\left(\Phi_{0}^{l}\right)^{-1} D_{l} \Phi_{0}^{l}=M^{l} \text { with } \\
& D_{r}=\left(v_{a} \mathrm{v}_{r}^{-1}\right) \sigma_{r}\left(\mathrm{v}_{r}\right) \quad, \quad D_{l}=\left(v_{a}^{-1} \mathrm{v}_{l}\right) \sigma_{l}\left(\mathrm{v}_{l}^{-1}\right) .
\end{aligned}
$$

Here, $\mathrm{v}_{\alpha} \in \mathcal{C}^{\alpha}$ are images of the ribbon element $v \in \mathcal{G}$ under the canonical isomorphisms from the center of $\mathcal{G}$ into the subalgebras $\mathcal{C}^{\alpha} \subset \mathcal{K}_{N}$ (cf. eq. (2.23)). The latter are generated by quantum traces of monodromies, i.e., by elements of the form $\operatorname{tr}_{q}^{I} \tau^{I}\left(M^{\alpha}\right)$ (for notations see Subsection 4.3). The elements $\mathcal{R}_{ \pm}^{\alpha}$ in eqs. (5.13) are given through the standard formula $\mathcal{R}_{ \pm}^{\alpha}=F_{\alpha}^{\prime} R_{ \pm}\left(F_{\alpha}\right)^{-1} \in \mathcal{G}_{a} \otimes \mathcal{G}_{a} \otimes \mathcal{C}^{\alpha}$.

5.2 Vertex operators at different sites. Definition 3 involves only vertex operators assigned to the $0^{\text {th }}$ site of the lattice. We may now try to construct vertex operators $\Phi_{n}^{\alpha} \in \mathcal{G}_{a} \otimes \mathcal{W}_{N}$ from elements in the algebra $\mathcal{W}_{N}$ which are assigned to other sites $n \neq 0$. 
In particular, they are required to satisfy the characteristic fusion and braid relations of vertex operators and, moreover, we want them to commute with all elements in $\mathcal{K}_{N}$ which are assigned to sites $m \neq n$ or edges $m \neq n, n+1$. The solution to this problem is certainly not unique. In the following, we shall describe just one possible construction. The idea is to introduce the vertex operators $\Phi_{n}^{\alpha}$ with the help of the holonomies $U_{n}^{\alpha} \in \mathcal{J}_{N}^{\alpha}$ by the simple formulae:

$$
\Phi_{n}^{r}:=\Phi_{0}^{r} U_{n}^{r} \quad, \quad \Phi_{n}^{l}:=\Phi_{0}^{l} U_{n}^{l} \quad \text { for } \quad n=1, \ldots, N-1 .
$$

Using the relations 4.12)-(4.16) for chiral holonomies, it is easy to verify the following properties of $\Phi_{n}^{\alpha}$ :

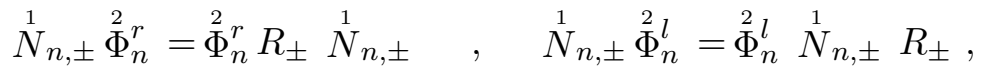

$$
\begin{aligned}
& \left(\Phi_{n}^{r}\right)^{*}=\left(S_{n}\right)^{-1}\left(\Phi_{n}^{r}\right)^{-1} \quad, \quad\left(\Phi_{n}^{l}\right)^{*}=S_{n}\left(\Phi_{n}^{l}\right)^{-1}, \\
& \iota_{n}(\xi) \Phi_{n}^{r}=\Phi_{n}^{r} \Delta_{n}^{\prime}(\xi) \quad, \quad \iota_{n}(\xi) \Phi_{n}^{l}=\Phi_{n}^{l} \Delta_{n}(\xi) \text { for all } \xi \in \mathcal{G},
\end{aligned}
$$

and $\iota_{m}(\xi)$ commute with $\Phi_{n}^{\alpha}$ for any $m \neq n$. Here $S_{n}=\left(i d \otimes \iota_{n}\right)(S)$ with $S \in \mathcal{G}_{a} \otimes \mathcal{G}$ as before. Next, one has to investigate fusion and braiding properties of $\Phi_{n}^{\alpha}$. The computation (see Appendix A.5) reveals that the elements $\Phi_{n}^{\alpha}$ obey the same relations as our vertex operators $\Phi_{0}^{\alpha}$ at the $0^{\text {th }}$ site, i.e.

$$
\begin{aligned}
& \stackrel{2}{\Phi}_{n}^{r} \stackrel{1}{\Phi}{ }_{n}^{r}=F_{r} \Delta_{a}\left(\Phi_{n}^{r}\right) \quad, \quad \stackrel{1}{\Phi}_{n}^{l} \stackrel{2}{\Phi}{ }_{n}^{l}=F_{l} \Delta_{a}\left(\Phi_{n}^{l}\right),
\end{aligned}
$$

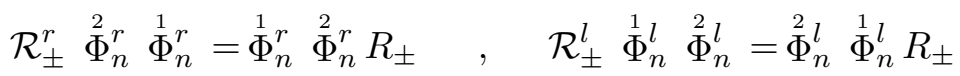

$$
\begin{aligned}
& \Phi_{n}^{\alpha} \mathrm{f}_{\alpha}=\sigma_{\alpha}\left(\mathrm{f}_{\alpha}\right) \Phi_{n}^{\alpha} \quad \text { for all } \mathrm{f}_{\alpha} \in \mathcal{C}^{\alpha}, \alpha=l, r
\end{aligned}
$$

hold with structure data $F_{\alpha}, \mathcal{R}_{ \pm}^{\alpha}, \sigma_{\alpha}$ being identical to the structure data of $\Phi_{0}^{\alpha}$ in eqs. (5.1), (5.2) and (5.13). In order to get an analogue of eqs. (5.14), we introduce the monodromies

$$
M_{n}^{\alpha}:=J_{n+1}^{\alpha} \ldots J_{N}^{\alpha} J_{1}^{\alpha} \ldots J_{n}^{\alpha}=\left(U_{n}^{\alpha}\right)^{-1} M^{\alpha} U_{n}^{\alpha} \quad \text { for } \quad \alpha=r, l .
$$

They are holonomies along the whole circle which begin and end at the $n^{\text {th }}$ site. It is now obvious that

$$
v_{a}^{-1}\left(\Phi_{n}^{r}\right)^{-1} D_{r} \Phi_{n}^{r}=M_{n}^{r} \quad, \quad v_{a}\left(\Phi_{n}^{l}\right)^{-1} D_{l} \Phi_{n}^{l}=M_{n}^{l}
$$

hold for all $0 \leq n<N$ and the elements $D_{\alpha} \in \mathcal{G}_{a} \otimes \mathcal{C}^{\alpha}$ are the same as in eqs. (5.14),(5.15). Let us remark that the quantum traces $\operatorname{tr}_{q}^{I} \tau^{I}\left(M_{n}^{\alpha}\right)$ are elements of the algebras $\mathcal{C}^{\alpha} \subset \mathcal{K}_{N}$ from which we constructed our structure data. Moreover, they do not depend on the index $n$, i.e., one can prove that $\operatorname{tr}_{q}^{I} \tau^{I}\left(M_{n}^{\alpha}\right)=\operatorname{tr}_{q}^{I} \tau^{I}\left(M_{m}^{\alpha}\right)$ for all pairs $n, m$ [7].

It still remains to investigate the exchange relations of the vertex operators $\Phi_{n}^{\alpha}$ with currents $J_{n}^{\alpha} \in \mathcal{G}_{a} \otimes \mathcal{K}_{N}$. Details are explained in Appendix A.5; here we only state the results:

$$
\begin{aligned}
& \stackrel{1}{J_{n+1}^{r}} \stackrel{\stackrel{2}{\Phi}}{n}=\stackrel{2}{\Phi_{n}^{r}} R_{+} \stackrel{1}{J_{n+1}^{r}} \quad, \quad \stackrel{2}{\Phi}{ }_{n}^{r} \stackrel{1}{J_{n}^{r}}=\stackrel{1}{J_{n}^{r}} \stackrel{2}{\Phi}{ }_{n}^{r} R_{-},
\end{aligned}
$$

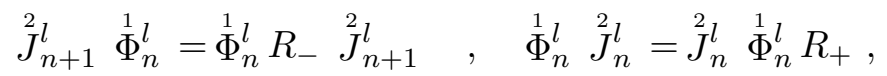

$$
\begin{aligned}
& \stackrel{1}{\Phi}_{n}^{r} \quad \stackrel{2}{J}_{m}^{r}=\stackrel{2}{J_{m}^{r}} \stackrel{\stackrel{1}{\Phi}}{n} \quad, \quad \stackrel{1}{\Phi}_{n}^{l} \quad \stackrel{2}{J_{m}^{l}}=\stackrel{2}{J_{m}^{l}} \stackrel{\stackrel{1}{\Phi}}{l} \quad \text { for } m \neq n, n+1,
\end{aligned}
$$

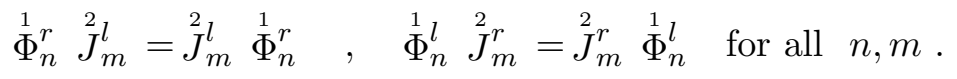


Finally, as a consequence of these relations and (5.3) we derive that

$$
\stackrel{1}{\Phi}_{n}^{r} \stackrel{2}{\Phi}=\stackrel{2}{m}=\stackrel{1}{\Phi} \stackrel{1}{\Phi}_{n}^{r} \quad \text { for all } n, m
$$

To summarize, we established that the construction (5.16) provides us with chiral vertex operators $\Phi_{n}^{r}$ and $\Phi_{n}^{l}$ which are naturally assigned to the $n^{t h}$ site of the lattice. These vertex operators share the same structure data $F_{r}, \mathcal{R}_{ \pm}^{r}, \ldots$ and $F_{l}, \mathcal{R}_{ \pm}^{l}, \ldots$. Their exchange relations with elements of the current algebra $\mathcal{K}_{N}$ are local in the sense discussed above.

Although the vertex operators have local relations with the observables, one should expect that they themselves are non-local. Indeed, it is easy to derive the following exchange relations (see Appendix A.5):

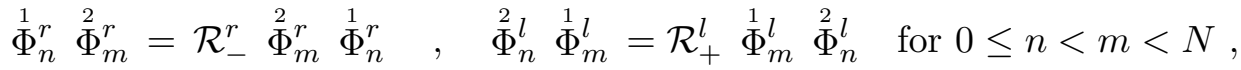

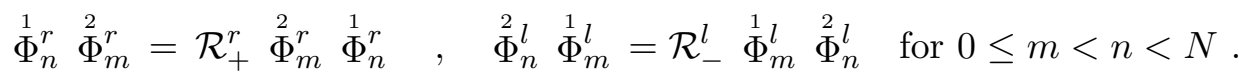

So, elements $\Phi_{n}^{\alpha}$ and $\Phi_{m}^{\alpha}$ do not commute even if the $n^{t h}$ and $m^{\text {th }}$ site at which they are localized are far apart. The relations (5.30), (5.31) demonstrate clearly that $\mathcal{R}_{ \pm}^{\alpha}$ play the role of braiding matrices in local quantum field theory.

5.3 Extension on a covering of the circle. In Subsection 5.2 we have listed properties of the vertex operators $\Phi_{n}^{\alpha}$ which are valid for $0 \leq n, m<N$. However, unlike the generators of $\mathcal{K}_{N}$, the vertex operators live on a covering of the circle, i.e., if we want to make sense of objects $\Phi_{n}^{\alpha}$ with $n \in \mathbb{Z}$, the operator $\Phi_{n+N}^{\alpha}$ necessarily differs from $\Phi_{n}^{\alpha}$. Indeed, $\Phi_{n}^{\alpha}$ may be defined for $n \in \mathbb{Z}$ by the following difference equation which is encoded in eqs. (5.16):

$$
\Phi_{n+1}^{\alpha}=\Phi_{n}^{\alpha} J_{n+1}^{\alpha}
$$

Here we assume that $J_{n}^{\alpha}$ has been extended periodically to $n \in \mathbb{Z}$. Periodicity properties of the objects $\Phi_{n}^{\alpha}$ can be expressed through the monodromies $M_{n}^{\alpha}$ introduced in (5.23),

$$
\Phi_{n+k N}^{\alpha}=\Phi_{n}^{\alpha}\left(M_{n}^{\alpha}\right)^{k}, \quad 0 \leq n<N, \quad k \in \mathbb{Z} .
$$

To proceed, we observe that properties of $M_{n}^{\alpha}$ are similar to those of $M^{\alpha} \equiv M_{0}^{\alpha}$. Using relations spelled out in Section 4, we easily find that $M_{n}^{\alpha}$ obey the functorial relations

$$
\stackrel{2}{M}_{n}^{r} R_{+} \stackrel{1}{M} r=R_{-} \Delta_{a}\left(M_{n}^{r}\right), \quad \stackrel{1}{M}_{n}^{l} R_{-} \stackrel{2}{M}_{n}^{l}=R_{+} \Delta_{a}\left(M_{n}^{l}\right)
$$

which coincide with (4.19). Therefore, $M_{n}^{r}$ and $M_{n}^{l}$ obey the exchange relations (3.2) and (3.7). Bearing this in mind, we employ (4.21) to derive

$$
\stackrel{2}{\Phi_{m}^{r}} R_{+} \stackrel{1}{M_{n}^{r}}=\stackrel{1}{M}_{n}^{r} \stackrel{2}{\Phi}{ }_{m}^{r} R_{-}, \quad \stackrel{1}{\Phi}{ }_{m}^{l} R_{-} \stackrel{2}{M}_{n}^{l}=\stackrel{2}{M_{n}^{l}} \stackrel{\stackrel{1}{\Phi}_{m}^{l}}{R_{+}}
$$

for $0 \leq n<N$ and $m=n(\bmod N)$, i.e., $m=n+k N, k \in \mathbb{Z}$.

Using the properties of the monodromies $M_{n}^{\alpha}$, we can establish (see Appendix A.5) that relations (5.18)-(5.22), (5.24)-(5.28) are valid for $\Phi_{n}^{\alpha}$ with the coordinate $n$ being replaced by $n^{\prime}=n+k N$. Thus, the local properties of vertex operators $\Phi_{n+k N}^{\alpha}$ living outside of the interval $0 \leq n<N$ coincide with those of $\Phi_{n}^{\alpha}$ living inside this interval.

The extension of the exchange relations between vertex operators to the covering of our discrete circle is slightly more subtle. For instance, the braid relation of the vertex 
operator $\Phi_{n}^{\alpha}$ and its counterpart $\Phi_{n+N}^{\alpha}$ does not coincide with 5.21). Instead, we find (see Appendix A.5):

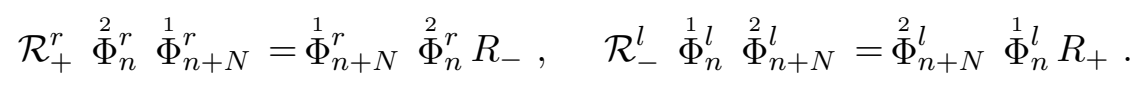

A similar situation is found for the braid relations (5.30)-(5.31). It turns out that here we need to apply eqs. (2.25) for the structure data of the vertex operators. Let us demonstrate their role by investigating the first eq. in (5.30) (i.e, the case $n<m$ ) with $n$ replaced by $n+N$,

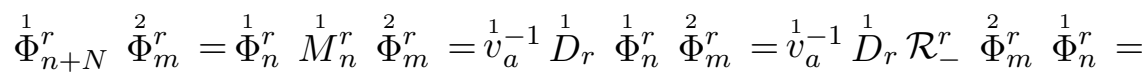

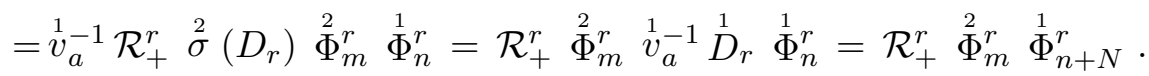

We see that the result coincides with the first eq. in (5.31), which is natural since $n+N>$ $m$. Proceeding in the same way, one can show that the braid relations (5.30) and (5.31) hold, for all $n, m \in \mathbb{Z}$ such that $|n-m|<N, n \neq m$. Thus, the equations (2.25) became an important ingredient for a self-consistent extension of the lattice theory beyond the interval $0 \leq n<N$.

5.4 Construction of the local field $g_{n}$. As we have shown above, the local properties of lattice vertex operators are the same as those we studied in the toy model case. Therefore, we can repeat the construction of Subsection 3.4 and introduce the objects

$$
g_{n}:=\mathcal{S}_{a}\left(\Phi_{n}^{l}\right) \Phi_{n}^{r} \in \mathcal{G}_{a} \otimes \mathcal{W}_{N}
$$

To proceed, we need some more information about the representation theory of lattice current algebras. As we mentioned before, the algebras $\mathcal{K}_{N}$ admit a series of irreducible representations on spaces $W_{N}^{I J}$ where $I, J$ run through classes of irreducible representations of the quantum algebra $\mathcal{G}$. These spaces $W_{N}^{I J}$ are of the form

$$
W_{N}^{I J}=V^{I} \otimes V^{J} \otimes \Re^{\otimes_{N-1}} \quad \text { where } \quad \Re=\bigoplus_{K} V^{\bar{K}} \otimes V^{K}
$$

Suppose that we describe $\mathcal{K}_{N}$ in terms of the holonomies $U_{n}^{\alpha}, n=1, \ldots, N-1$, the monodromies $M_{0}^{\alpha}$ and the local elements $N_{n}, n=0, \ldots, N-1$ (notice that the currents can be reconstructed from holonomies and monodromies). We divide these generators into two sets, the first containing all $U_{n}^{\alpha}$ and $N_{m}$ for $m \neq 0$ while we put $M_{0}^{\alpha}$ and $N_{0}$ into the second set. This choice is made so that objects which were not part of the toy model are separated from objects we met in Section 3 already. In [6], an action of $\mathcal{K}_{N}$ on $W_{N}^{I J}$ was constructed for which objects in the first set, i.e., holonomies $U_{n}^{\alpha}$ and elements $N_{m}, m \neq 0$, act trivially on the factor $V^{I} \otimes V^{J}$ in $W_{N}^{I J}$ and irreducibly on $\Re^{\otimes_{N-1}}$.

It is then straightforward to see that our algebra $\mathcal{W}_{N}$ of vertex operators on the lattice possesses only one irreducible representation on the total space

$$
\mathcal{M}_{N}=\bigoplus_{I, J} W_{N}^{I J} \cong \mathcal{M} \otimes \Re^{\otimes_{N-1}}
$$

where each summand $W_{N}^{I J}$ appears with multiplicity one. By now, the picture resembles very much the situation in the toy model: we have the model space $\mathcal{M}_{N}$ on which $\mathcal{W}_{N}$ acts 
irreducibly. Therefore, we may look for operators that can be restricted to the diagonal subspace

$$
\mathcal{H}_{N}=\bigoplus_{J} W_{N}^{\bar{J} J} \cong \mathcal{H} \otimes \Re^{\otimes_{N-1}} \subset \mathcal{M}_{N}
$$

This is certainly possible for all elements in $\mathcal{K}_{N}$. But in addition, we may restrict the field $g_{n}$ to $\mathcal{H}_{N}$. As in Subsection 3.4, the diagonal subspace is characterized by the constraint $\mathrm{f}=\mathcal{S}_{l r}(\mathrm{f})$ for all $\mathrm{f} \in \mathcal{C}^{r} \subset \mathcal{K}_{N}$. If we adjust left and right structure data according to eqs. (3.14) [4], the constraint to $\mathcal{H}_{N}$ is compatible with the construction of $g_{n}$, i.e., (3.17) holds with $g$ replaced by $g_{n}, n=0, \ldots, N-1$. The properties of the restricted field are spelled out in the following proposition.

Proposition 5 (Properties of $g_{n}$ ) When restricted to the diagonal subspace $\mathcal{H}_{N}$, the element $g_{n} \in \mathcal{G}_{n} \otimes \operatorname{End}\left(\mathcal{H}_{N}\right)$ obeys the following relations:

$$
\begin{aligned}
& \stackrel{2}{g}_{n} \stackrel{1}{g}_{n}=\Delta_{a}\left(g_{n}\right) \quad, \quad R_{ \pm} \stackrel{2}{g}_{n} \stackrel{1}{g}_{n}=\stackrel{1}{g}_{n} \stackrel{2}{g}_{n} R_{ \pm} \\
& M_{n}^{l} g_{n}=g_{n} M_{n}^{r} \quad, \quad \mathcal{S}_{a}\left(g_{n}\right)=g_{n}^{-1}, \\
& g_{n+N}=g_{n} \quad, \quad \stackrel{1}{g}_{n} \stackrel{2}{g}_{m}=\stackrel{2}{g}_{m} \stackrel{1}{g}_{n} \quad \text { for } n \neq m, \\
& \stackrel{1}{M}_{n}^{r} \stackrel{2}{g}_{n} \quad R_{-}=\stackrel{2}{g}_{n} \quad R_{+} \stackrel{1}{M}_{n}^{r} \quad, \quad \stackrel{1}{M}_{n}^{l} R_{-} \stackrel{2}{g}_{n}=R_{+} \stackrel{2}{g}_{n} \stackrel{1}{M}_{n}^{l}, \\
& \Delta_{n}(\xi) g_{n}=g_{n} \Delta_{n}^{\prime}(\xi) \quad, \quad \stackrel{1}{N}_{n, \pm} R_{ \pm} \stackrel{2}{g}_{n}=\stackrel{2}{g}_{n} R_{ \pm} \stackrel{1}{N}{ }_{n, \pm}
\end{aligned}
$$

for all $\xi \in \mathcal{G}$ and $g_{n}$ commutes with all $\iota_{m}(\xi) \in \mathcal{G}_{n} \subset \mathcal{K}_{N}$ for $m \neq n$.

The properties listed above, and in particular the locality and periodicity relations (5.40), allow to regard $g_{n}$ as an observable in the lattice WZNW-model. It is a discrete analogue of the group valued field $g(x)$. Some remarks on the proof of Proposition 5 can be found in Appendix A.6. To complete the description of $g_{n}$, let us give its exchange relations with the chiral currents. Using (5.25), we obtain

$$
\begin{aligned}
& \stackrel{2}{g}_{n} \stackrel{1}{J}_{n}^{r}=\stackrel{1}{J}_{n}^{r} \stackrel{2}{g}_{n} R_{-} \quad, \quad \stackrel{2}{g}_{n} \stackrel{1}{J} l_{n}^{l}=\stackrel{1}{J_{n}^{l}} R_{-} \stackrel{2}{g}_{n} \\
& \stackrel{1}{J}_{n+1}^{r} \stackrel{2}{g}_{n}=\stackrel{2}{g}_{n} R_{+} \stackrel{\stackrel{1}{J}_{n+1}^{r}}{,} \quad, \quad \stackrel{1}{J}_{n+1}^{l} \stackrel{2}{g}_{n}=R_{+} \stackrel{2}{g}_{n} \stackrel{1}{J}_{n+1}^{l}, \\
& \stackrel{1}{J}_{m}^{\alpha} \stackrel{2}{g}_{n}=\stackrel{2}{g}_{n} \stackrel{1}{J}_{m}^{\alpha}, \alpha=l, r \quad \text { for } m \neq n, n+1(\bmod N) \text {. }
\end{aligned}
$$

5.5 Lattice vertex operators for $\mathcal{Z}_{q}$. Let us construct the algebra $\mathcal{W}_{N}$ in the case of $\mathcal{G}=\mathcal{Z}_{q}$. To this end we have to add the chiral vertex operators introduced in Subsection 3.5 to the lattice $U(1)$-current algebra discussed in Subsection 4.4. As a result we get the algebra generated by components of the following elements belonging to $\mathcal{G}_{a} \otimes \mathcal{W}_{N}$ :

$$
\begin{gathered}
\Phi_{n}^{\alpha}=\sum_{s=0}^{p-1} P^{s} \otimes\left(\widehat{Q}_{n}^{\alpha}\right)^{s}=e^{\widehat{p} \otimes \widehat{\varsigma}_{n}^{\alpha}} \quad, \quad N_{n, \pm}=\sum_{s=0}^{p-1} P^{s} \otimes h_{n}^{ \pm s}=q^{ \pm \widehat{p} \otimes \widehat{p}_{n}}, \\
J_{n}^{r}=\sum_{s=0}^{p-1} q^{\frac{1}{2} s^{2}} P^{s} \otimes\left(\widehat{W}_{n}^{r}\right)^{s}=k_{a}^{-\frac{1}{2}} e^{\widehat{p} \otimes \widehat{\varpi}_{n}^{r}} \quad, \quad J_{n}^{l}=\sum_{s=0}^{p-1} q^{-\frac{1}{2} s^{2}} P^{s} \otimes\left(\widehat{W}_{n}^{l}\right)^{s}=k_{a}^{\frac{1}{2}} e^{\widehat{p} \otimes \widehat{\varpi}_{n}^{l}}
\end{gathered}
$$

\footnotetext{
${ }^{14}$ This can be done simultaneously for all sites, since the structure data do not depend on the lattice site $n$ (see Subsection 5.2).
} 
where $\alpha=r, l$ and $n=0, . ., N-1$. According to eqs. (4.6) and (5.16), not all the generators are independent. Namely, the following relations are to be fulfilled:

$$
\widehat{W}_{n}^{l}=h_{n-1}^{-1} \widehat{W}_{n}^{r} h_{n}^{-1}, \quad \widehat{Q}_{n}^{\alpha}=\widehat{Q}_{0}^{\alpha} \widehat{W}_{1}^{\alpha} \ldots \widehat{W}_{n}^{\alpha} .
$$

Due to the Campbell-Hausdorff formula these equalities may be re-expressed in terms of the generators $\widehat{\varpi}_{n}^{\alpha}, \widehat{\varsigma}_{n}^{\alpha}$ as follows:

$$
\widehat{\varpi}_{n}^{l}=\widehat{\varpi}_{n}^{r}-\ln q\left(\widehat{p}_{n}+\widehat{p}_{n-1}\right), \quad \widehat{\varsigma}_{n}^{\alpha}=\widehat{\varsigma}_{0}^{\alpha}+\sum_{k=1}^{n} \widehat{\varpi}_{k}^{\alpha} .
$$

It is easy to see that all the formulae between $\Phi_{n}^{\alpha}, J_{n}^{\alpha}$ and $N_{n, \pm}$ spelled out in Subsections 5.1-5.3 are satisfied if we add to eqs. (4.25)-4.26) or, alternatively, to eqs. (4.27) the following relations:

$$
\begin{aligned}
h_{n} \widehat{Q}_{n}^{\alpha}=q \widehat{Q}_{n}^{\alpha} h_{n}, & h_{m} \widehat{Q}_{n}^{\alpha}=\widehat{Q}_{n}^{\alpha} h_{m} \text { for } m \neq n, \\
\widehat{W}_{n+1}^{r} \widehat{Q}_{n}^{r}=q \widehat{Q}_{n}^{r} \widehat{W}_{n+1}^{r}, & \widehat{W}_{n}^{r} \widehat{Q}_{n}^{r}=q \widehat{Q}_{n}^{r} \widehat{W}_{n}^{r}, \\
\widehat{W}_{n+1}^{l} \widehat{Q}_{n}^{l}=q-1 \widehat{Q}_{n}^{l} \widehat{W}_{n+1}^{l}, & \widehat{W}_{n}^{l} \widehat{Q}_{n}^{l}=q^{-1} \widehat{Q}_{n}^{l} \widehat{W}_{n}^{l}, \\
\widehat{W}_{m}^{\alpha} \widehat{Q}_{n}^{\alpha}=\widehat{Q}_{n}^{\alpha} \widehat{W}_{m}^{\alpha} & \text { for } m \neq n, n+1,
\end{aligned}
$$

which can be rewritten as follows:

$$
\left[\widehat{p}_{m}, \widehat{\varsigma}_{n}^{\alpha}\right]=\delta_{m, n}, \quad\left[\widehat{\varpi}_{m}^{r}, \widehat{\varsigma}_{n}^{r}\right]=-\left[\widehat{\varpi}_{m}^{l}, \widehat{\varsigma}_{n}^{l}\right]=\ln q\left(\delta_{m, n+1}+\delta_{m, n}\right)
$$

Since we already discussed properties of the vertex operators at a fixed site for the $\mathcal{Z}_{q^{-}}$ theory in Subsections 2.6 and 3.5, we shall concentrate on the aspects of locality and periodicity here. Actually, the latter simplify in the case of $\mathcal{Z}_{q}$ due to the circumstance that all our monodromies $M_{n}^{\alpha}$ of the same chirality coincide (since all they are given by $(4.29))$. This allows to rewrite eqs. (5.30)-(5.31) and (5.36) in the form (recall that in the case of $\mathcal{Z}_{q}$ we have $\mathcal{R}_{ \pm}=R_{ \pm}=R^{ \pm 1}$ with $R$ given in Subsections 2.1 and 2.2):

$$
\stackrel{1}{\Phi}_{n}^{r} \stackrel{2}{\Phi}=R_{m}^{\beta(n-m)} \stackrel{2}{\Phi}{ }_{m}^{r} \stackrel{1}{\Phi}{ }_{n}^{r}, \quad \stackrel{2}{\Phi_{n}^{l}} \stackrel{1}{\Phi}{ }_{m}^{l}=R^{-\beta(n-m)} \stackrel{1}{\Phi}{ }_{m}^{l} \stackrel{2}{\Phi}{ }_{n}^{l}
$$

for all $n, m \in \mathbb{Z}$. Here $\beta(n-m)=1+2\left[\frac{n-m}{N}\right]([x]$ stands for the entire part of $x)$ for $n \neq$ $m(\bmod N)$ and $\beta(n-m)=1+\left[\frac{n-m}{N}\right]$ for $n=m(\bmod N)$. In the derivation we have also used the following consequence of eqs. (5.45): $\left[\sum_{k=1}^{N} \widehat{\varpi}_{k}^{r}, \widehat{\varsigma}_{n}^{r}\right]=-\left[\sum_{k=1}^{N} \widehat{\varpi}_{k}^{l}, \widehat{\varsigma}_{n}^{l}\right]=2 \ln q$. Notice that, since $R^{p}=e \otimes e$, the above relations are actually periodic with a period $N^{\prime}=p N$ for odd $p$. That is, the theory lives on a $p$-fold covering of the circle so that vertex operators for $\mathcal{Z}_{q}$ are periodic on a lattice of size $N^{\prime}=p N$.

Now let us introduce the field $g_{n}$. We repeat the construction of Subsection 3.5 and define $g_{n}$ as follows:

$$
g_{n}=\sum_{s=0}^{p-1} P^{s} \otimes\left(\widehat{Q}_{n}^{r}\right)^{s}\left(\widehat{Q}_{n}^{l}\right)^{-s}=e^{\widehat{p} \otimes\left(\widehat{\varsigma}_{n}^{r}-\widehat{\varsigma}_{n}^{l}\right)} .
$$

It obviously admits restriction to the diagonal subspace $\mathcal{H}_{N}$ of the model space $\mathcal{M}_{N}$ (cf. Subsections 3.5 and 5.4). The locality of $g_{n}$ is evident from eqs. (5.46) and its periodicity $g_{n+N}=\left(M^{l}\right)^{-1} g_{n} M^{r}=g_{n}$ is, in fact, reduced to the Weyl-type relation which we explained in detail at the end of Subsection 3.5. 


\section{AUTOMORPHISMS AND DISCRETE DYNAMICS}

In this section we shall demonstrate that the lattice theory which we constructed above indeed may be regarded as a discretization of the WZNW model. For this purpose we investigate the exchange relations of currents and some automorphisms of our lattice algebra in the classical continuum limit and recover the Poisson structure and the dynamics of the classical WZNW model, respectively.

6.1 Remarks on the classical continuum limit. Let us briefly discuss the classical continuum limit of the algebra of vertex operators. Following ideology of [4], we rewrite the exchange relations (4.2), (4.4) and (4.7), (4.8) for the chiral currents in a more compact form:

$$
\begin{aligned}
& R_{n-m,+}^{-1} \stackrel{2}{J_{n}^{r}} R_{n-m+1,+} \stackrel{1}{J}{ }_{m}^{r}=\stackrel{1}{J}_{m}^{r} R_{n-m-1,-}^{-1} \stackrel{2}{J_{n}^{r}} R_{n-m,-}, \\
& R_{n-m,+}^{-1} \stackrel{1}{J_{n}^{l}} R_{n-m+1,-} \stackrel{2}{J}_{m}^{l}=\stackrel{2}{J}_{m}^{l} R_{n-m-1,+}^{-1} \stackrel{1}{J}_{n}^{l} R_{n-m,-},
\end{aligned}
$$

where $R_{n, \pm}:=\delta_{n, 0} R_{ \pm}+\left(1-\delta_{n, 0}\right) e \otimes e$ is, as usual, an element of $\mathcal{G}_{a} \otimes \mathcal{G}_{a}$. Now we consider these relations in the limit where $a=2 \pi / N \rightarrow 0$ and $\hbar \rightarrow 0$.

Since for our theory $q=\exp \{i \gamma \hbar\}$ (cf. Introduction), we can expand the universal $R$-matrix according to $R_{ \pm}=e \otimes e+i \gamma \hbar r_{ \pm}+O\left(\hbar^{2}\right)$. On the other hand, the lattice fields approach their continuum counterparts as $a$ becomes small:

$$
J_{n}^{\alpha} \rightarrow e \otimes \mathrm{e}-a j^{\alpha}(x), \quad \Phi_{n}^{\alpha} \rightarrow \Phi^{\alpha}(x), \quad g_{n} \rightarrow g(x),
$$

where $x=a n$. Bearing in mind that $\frac{1}{a} \delta_{n, 0} \rightarrow \delta(x)$ when $a \rightarrow 0$, we obtain the following Poisson brackets from (6.1)-(6.2):

$$
\begin{aligned}
\left\{j^{1}(x), j^{r}(y)\right\} & =\frac{\gamma}{2}\left[C,{ }_{j}^{r}(x)-\stackrel{2}{j}^{r}(y)\right] \delta(x-y)+\gamma C \delta^{\prime}(x-y), \\
\left\{j^{l}(x),{ }_{j}^{2} l(y)\right\} & =-\frac{\gamma}{2}\left[C,{ }_{j}^{l} l(x)-\stackrel{2}{j}^{l}(y)\right] \delta(x-y)-\gamma C \delta^{\prime}(x-y),
\end{aligned}
$$

where $C=\left(r_{+}-r_{-}\right) \otimes$ e. These are the standard brackets for the chiral WZNW currents [41, 51], and the deformation parameter $\gamma$ is identified with the coupling constant. 15

The exchange relations (5.25)-(5.26) can be treated similarly. Namely, we rewrite them as follows

$$
\stackrel{1}{J_{n}^{r}} \stackrel{2}{\Phi}{ }_{m}^{r} R_{n-m,-}=\stackrel{2}{\Phi}{ }_{m}^{r} R_{n-m-1,+} \stackrel{1}{J_{n}^{r}}, \quad \stackrel{2}{J}{ }_{n}^{l} \stackrel{1}{\Phi}{ }_{m}^{l} R_{n-m,+}=\stackrel{1}{=}{ }_{m}^{l} R_{n-m-1,-} \stackrel{2}{J}{ }_{n}^{l},
$$

and get the following Poisson brackets for vertex operators in the classical continuum limit:

$$
\left\{\stackrel{j}{j}^{r}(x), \stackrel{2}{\Phi}^{r}(y)\right\}=\gamma \stackrel{2}{\Phi}^{r}(x) C \delta(x-y), \quad\left\{{ }_{j}^{1} l(x), \stackrel{2}{\Phi}^{l}(y)\right\}=-\gamma \stackrel{2}{\Phi}^{l}(x) C \delta(x-y) .
$$

These relations are classical counterparts of the commutation relations known for the chiral primary fields in the continuum WZNW model [41].

Substitution of the expansions $\mathcal{R}_{ \pm}^{\alpha}=e \otimes e \otimes \mathrm{e}+i \gamma \hbar \mathbf{r}_{ \pm}^{\alpha}+O\left(\hbar^{2}\right)$ into eqs. (5.21) and passing to the classical continuum limit gives 10

$\left\{\stackrel{1}{\Phi}^{r}(x), \stackrel{2}{\Phi}^{r}(y)\right\}=-\chi^{r}(x-y) \stackrel{1}{\Phi} r(x) \stackrel{2}{\Phi}^{r}(y), \quad\left\{\stackrel{1}{\Phi}^{l}(x) \stackrel{2}{\Phi}^{l}(y)\right\}=\chi^{l}(x-y) \stackrel{1}{\Phi} l(x) \stackrel{2}{\Phi}^{l}(y)$,

\footnotetext{
${ }^{15}$ One may prefer to renormalize the currents by $1 / \gamma$ so that the $\delta^{\prime}$-term acquires a coefficient $1 / \gamma$ which, in the classical theory, coincides with the level $k$ of the KM algebra. The quantum correction $1 / \gamma \rightarrow k+\nu$ is explained, e.g., in [t].

${ }^{16}$ In general, the classical $r$-matrices $\mathbf{r}_{ \pm}^{\alpha}$ keep a non-trivial dependence on variables belonging to $\mathcal{C}^{\alpha}$.
} 
where $\chi^{\alpha}(x-y)=\varepsilon(x-y) \gamma \mathbf{r}_{+}^{\alpha}+\varepsilon(y-x) \gamma \mathbf{r}_{-}^{\alpha}$, and $\varepsilon(x)=1$ if $x>0$ and $\varepsilon(x)=0$ if $x<0$. Such brackets were obtained for the classical WZNW model in [26, 13, 20, 27].

The same technique may finally be applied to the relations (5.43) involving the lattice field $g$ and the resulting formulae for the classical counterpart of eqs. (5.43) coincide with formulae in 41], namely,

$$
\left\{{ }^{1} r(x), \stackrel{2}{g}(y)\right\}=\gamma \stackrel{2}{g}(x) C \delta(x-y), \quad\left\{{ }^{1} l(x), \stackrel{2}{g}(y)\right\}=\gamma C \stackrel{2}{g}(x) \delta(x-y) .
$$

Thus, in the limit $\hbar \rightarrow 0, a \rightarrow 0$, our main exchange relations for the chiral currents and chiral vertex operators reproduce the Poisson structure known for the classical WZNW model.

6.2 Automorphisms induced by the ribbon element. The ribbon element, due to its specific properties, allows to obtain certain inner automorphisms of the algebra $\mathcal{W}_{N}$. These are the subject of the present subsection.

Non-local automorphism induced by global ribbon elements. Consider an automorphism of the form:

$$
A \mapsto \mathrm{v}_{r}^{-1} \mathrm{v}_{l} A \mathrm{v}_{r} \mathrm{v}_{l}^{-1}, \quad \text { for all } A \in \mathcal{W}_{N}
$$

Here $\mathrm{v}_{r} \in \mathcal{C}^{r}$ and $\mathrm{v}_{l} \in \mathcal{C}^{l}$. We call the ribbon elements $\mathrm{v}_{\alpha}$ global because they are constructed from the monodromies $M^{\alpha}$, which are non-local.

Since the subalgebras $\mathcal{C}^{\alpha}$ constitute the center of $\mathcal{K}_{N}$, all the elements of the current algebra $\mathcal{K}_{N} \subset \mathcal{W}_{N}$ are invariant under the transformation (6.4). For the vertex operators this transformation is nontrivial and may be rewritten with the help of (5.15), (5.22) and (5.24) so that it becomes

$$
\Phi_{n+k N}^{\alpha} \mapsto \Phi_{n+k N}^{\alpha} M_{n}^{\alpha}=\Phi_{n+(k+1) N}^{\alpha}, \quad 0 \leq n<N, \quad k \in \mathbb{Z} .
$$

Thus, the automorphism (6.4) is non-local, i.e., it corresponds to a shift $n \mapsto n+N$ or, in other words, it rotates the lattice by angle $2 \pi$. Being restricted on the diagonal subspace, the field $g_{n}$ is periodic (see Proposition 5), and hence it is invariant under the transformation (6.4). In this sense, the automorphism (6.4) separates "physical" variables living on the circle from "non-physical" ones (like the vertex operators) living on a covering of the circle.

Local automorphism induced by local ribbon elements. Recall that the $n^{\text {th }}$ site of the lattice is supplied with a copy $\mathcal{G}_{n}$ of the symmetry algebra $\mathcal{G}$. Therefore we can use the local ribbon elements $v_{n} \in \mathcal{C}_{n} \subset \mathcal{G}_{n}$ to construct the following transformation:

$$
A \mapsto v_{0} v_{1} \ldots v_{N-1} A\left(v_{0} v_{1} \ldots v_{N-1}\right)^{-1}, \quad \text { for any } A \in \mathcal{W}_{N}
$$

Here the product is taken over all sites of the lattice.

To obtain more explicit formulae for the automorphism (6.5), we have to use relations (4.3), (4.10), (5.19), employ equation (2.1) and remember that $v_{n}$ belongs to the center of $\mathcal{G}_{n}$. As a result we get

$$
\begin{array}{ll}
J_{n}^{r} \mapsto N_{n-1} J_{n}^{r} N_{n}^{-1}, & J_{n}^{l} \mapsto \widetilde{N}_{n-1}^{-1} J_{n}^{l} \widetilde{N}_{n}, \\
\Phi_{n}^{r} \mapsto v_{a} \Phi_{n}^{r} N_{n}^{-1}, & \Phi_{n}^{l} \mapsto v_{a} \Phi_{n}^{l} \widetilde{N}_{n},
\end{array}
$$


where we used notations of Subsection 2.2, i.e., $N=N_{+} N_{-}^{-1}, \widetilde{N}=N_{+}^{-1} N_{-}$. The elements of $\mathcal{G}_{n}$ and $\mathcal{C}^{\alpha}$ remain invariant under (6.5), in particular, $N_{n, \pm} \mapsto N_{n, \pm}$. With the help of these explicit expressions we also obtain

$$
M_{n}^{r} \mapsto N_{n} M_{n}^{r} N_{n}^{-1}, \quad M_{n}^{l} \mapsto \tilde{N}_{n}^{-1} M_{n}^{l} \widetilde{N}_{n}, \quad g_{n} \mapsto \widetilde{N}_{n} g_{n} N_{n}^{-1}
$$

We know already that these formulae describe an automorphism of the algebra $\mathcal{W}_{N}$ because they were obtained by conjugation with a unitary element, namely the product of local ribbon elements, in formula (6.5). Without this knowledge, it would be a quite non-trivial task to check the automorphism property directly for the expressions in (6.6)-(6.7). To do this, one would need to apply the relations (4.5) and (5.17) many times.

Local automorphism induced by $\kappa_{n}$. To construct one more inner automorphism of $\mathcal{W}_{N}$ we employ the square roots of the local ribbon elements,

$$
A \mapsto \kappa_{0} \kappa_{1} \ldots \kappa_{N-1} A\left(\kappa_{0} \kappa_{1} \ldots \kappa_{N-1}\right)^{-1}, \quad \text { for all } A \in \mathcal{W}_{N}
$$

Here $\kappa_{n} \in \mathcal{C}_{n} \subset \mathcal{G}_{n}, \kappa_{n}^{2}=v_{n}$ and the product is taken over all sites.

Computations similar to those performed above (and making use of (2.10)) allow to rewrite the transformation $(6.8)$ in the following explicit form:

$$
\begin{array}{rlrl}
J_{n}^{r} & \mapsto N_{n-1,+}\left(S_{n-1}\right)^{-1} J_{n}^{r} S_{n} N_{n,+}^{-1} & =N_{n-1,+}\left(\left(J_{n}^{r}\right)^{*}\right)^{-1} N_{n,+}^{-1}, \\
J_{n}^{l} & \mapsto N_{n-1,-}^{-1} S_{n-1} J_{n}^{l}\left(S_{n}\right)^{-1} N_{n,-} & =N_{n-1,-}^{-1}\left(\left(J_{n}^{l}\right)^{*}\right)^{-1} N_{n,-}, \\
\Phi_{n}^{r} & \mapsto \kappa_{a} \Phi_{n}^{r} S_{n} N_{n,+}^{-1} & =\kappa_{a}\left(\left(\Phi_{n}^{r}\right)^{*}\right)^{-1} N_{n,+}^{-1}, \\
\Phi_{n}^{l} \mapsto \kappa_{a} \Phi_{n}^{l}\left(S_{n}\right)^{-1} N_{n,-} & =\kappa_{a}\left(\left(\Phi_{n}^{l}\right)^{*}\right)^{-1} N_{n,-} . \\
M_{n}^{r} \mapsto N_{n,+}\left(\left(M_{n}^{r}\right)^{*}\right)^{-1} N_{n,+}^{-1}, \quad M_{n}^{l} & \mapsto N_{n,-}^{-1}\left(\left(M_{n}^{l}\right)^{*}\right)^{-1} N_{n,-}
\end{array}
$$

and $N_{n, \pm} \mapsto N_{n, \pm}$, as before. Here the r.h.s. of all formulae have been rewritten with the help of the $*$-operation introduced for elements of $\mathcal{W}_{N}$ in Sections 4 and 5 . Having done so, we see that the image of all basic objects $X \in \mathcal{G}_{a} \otimes \mathcal{W}_{N}$ under the automorphism (6.8) coincides with $\left(X^{*}\right)^{-1}$ up to a multiplication with factors $N_{n, \pm}$. We can now accept an inverse logic - we may say that the automorphism $(6.8)$ together with the rules $\left(N_{ \pm}\right)^{*}=$ $N_{\mp}$ defines the $*$-operation on $\mathcal{W}_{N}$. This picture reveals the naturalness of our $*$-operation, which might have appeared somewhat artificial in the previous sections. It also makes the role of the ribbon element in our theory even more remarkable.

To conclude this discussion, we would like to mention that for a lattice of even length, i.e., for $N=0(\bmod 2)$, one may also consider automorphisms of $\mathcal{W}_{N}$ generated by the alternating products of $v_{n}^{ \pm 1}$ or $\kappa_{n}^{ \pm 1}$.

6.3 Discrete dynamics. As we saw above, the exchange relations of the algebra $\mathcal{W}_{N}$ allowed to recover the Poisson structure of the classical WZNW-model in the classical continuum limit. However, this is certainly not sufficient for a construction of the lattice WZNW model. Indeed, the complete description of a classical theory involves an evolution equation for the dynamical variables in addition to the specification of the Poisson structure. Similarly, the formulation of a discrete quantum model requires not only a set of exchange relations between quantum operators but also some one parameter family of automorphisms of the algebra generated by operators in the quantum theory. The parameter is interpreted as time variable. For a theory on a discrete space it is natural to discretize the time as well so that the parameter essentially runs through the set of integers only. In 
this case the whole family of automorphisms can be reconstructed from the automorphism which provides the evolution for an elementary step in time. Such an automorphism of a lattice model must be local, i.e., the result of its action on the variables assigned to a given site (or link) can only involve variables assigned to some neighboring sites (or links). In the previous subsection we considered three automorphisms of the algebra $\mathcal{W}_{N}$. The first of them was non-local and hence did not correspond to any dynamics. [T The second and the third automorphism were local and, in principle, one may use them in constructing the corresponding classical continuum models. However, the dynamics of such models do not reproduce the dynamics of the WZNW theory.

In this subsection we are going to consider local automorphisms which can be interpreted as dynamics of the discrete WZNW model. Let us recall that in the continuum WZNW model the equation of motion for the $G$-valued field $g(x)$ takes the form:

$$
\partial_{+} \partial_{-} g=\left(\partial_{+} g\right) g^{-1}\left(\partial_{-} g\right)
$$

where $\partial_{ \pm}=\frac{1}{2}\left(\partial_{0} \pm \partial_{x}\right)$. From the field $g(x)$ one may construct the following Lie algebra valued currents

$$
j^{r}=g^{-1} \partial_{-} g \quad, \quad j^{l}=\left(\partial_{+} g\right) g^{-1} .
$$

They turn out to be chiral objects in the sense that their equations of motion are trivialized:

$$
\partial_{+} j^{r}=\partial_{-} j^{l}=0 .
$$

In the Hamiltonian approach, the initial data are provided by the values of $g(0), j^{r}(x)$ and $j^{l}(x)$ at time $t=0$. To recover the dynamics of $g(x)$ one solves the equations

$$
\partial_{0} g=j^{l} g+g j^{r}, \quad \partial_{x} g=j^{l} g-g j^{r} .
$$

Eqs. (6.11) and (6.12) can be derived with the help of the Poisson brackets given in Subsection 6.1 if the Hamiltonian and the total momentum are chosen as follows:

$$
H=\frac{1}{2 \gamma} \int \operatorname{tr}\left[\left(j^{r}(x)\right)^{2}+\left(j^{l}(x)\right)^{2}\right] d x, \quad P=\frac{1}{2 \gamma} \int \operatorname{tr}\left[\left(j^{r}(x)\right)^{2}-\left(j^{l}(x)\right)^{2}\right] d x,
$$

where the integration is taken over the whole circle and $t r$ is the usual trace in the corresponding Lie algebra.

Let us develop an analogue of the given picture in the quantum lattice theory. More precisely, we shall consider the "physical" subalgebra $\mathcal{P}_{N}$ of $\mathcal{W}_{N}$ generated by components of the chiral currents $J_{n}^{\alpha}$ and the field $g_{n}, n=1, . ., N$ which are subject to the relations spelled out in Sections 4 and 5. As we have indicated in our general discussion above, it is natural to work with a discrete time with a minimal time interval $\tau$ (see also [28, 31]), so that the evolution of the quantum theory is described by a single automorphism of $\mathcal{P}_{N}$. In addition to this, we shall also introduce an automorphism which is responsible for the shifts by one lattice unit $a=2 \pi / N$ in space.

Lemma 2 (Shift and evolution automorphisms) Let $\mathcal{P}_{N}$ denote the algebra generated by components of $J_{n}^{\alpha}$ and $g_{n}$ (restriction to the diagonal subspace $\mathcal{H}_{N}$ is understood). Then the following two transformations $T_{V}, T_{U}$,

$$
\begin{aligned}
& T_{V}\left(J_{n}^{\alpha}\right)=J_{n+1}^{\alpha}, \quad \alpha=l, r, \\
& T_{V}\left(g_{n}\right)=\left(J_{n+1}^{l}\right)^{-1} g_{n} J_{n+1}^{r},
\end{aligned}
$$

\footnotetext{
${ }^{17}$ However, one can use it to describe dynamics of the toy model (see [3]).
} 
and

$$
\begin{aligned}
& T_{U}\left(J_{n}^{r}\right)=J_{n-1}^{r}, \quad T_{U}\left(J_{n}^{l}\right)=J_{n+1}^{l}, \\
& T_{U}\left(g_{n}\right)=\left(J_{n+1}^{l}\right)^{-1} g_{n}\left(J_{n}^{r}\right)^{-1}
\end{aligned}
$$

extend to automorphisms of the algebra $\mathcal{P}_{N}$. We call $T_{V}$ the shift automorphism and $T_{U}$ the evolution automorphism of the lattice $W Z N W$-model.

It is straightforward to verify that the transformations $T_{V}$ and $T_{U}$ preserve all the relations for $J_{n}^{\alpha}$ and $g_{n}$ given above. Notice also that one may extend $T_{U}, T_{V}$ to the whole algebra $\mathcal{W}_{N}$ by eqs. (6.14), (6.16) and, in addition, the formulae

$$
\begin{array}{ccc}
T_{V}\left(\Phi_{n}^{\alpha}\right) & =\Phi_{n}^{\alpha} J_{n+1}^{\alpha}, \\
T_{U}\left(\Phi_{n}^{r}\right)=\Phi_{n}^{r}\left(J_{n}^{r}\right)^{-1} & , \quad T_{U}\left(\Phi_{n}^{l}\right)=\Phi_{n}^{l} J_{n+1}^{l} .
\end{array}
$$

These automorphisms are actually combined of two chiral automorphisms (cf. Subsection 6.4). After restriction to the diagonal subspace and, hence, to the algebra $\mathcal{P}_{N}$, we recover eqs. (6.15) and (6.17). 18

Assume now that Lemma 2 describes inner automorphisms of $\mathcal{P}_{N}$. That is, suppose that there exist operators $\mathrm{V}, \mathrm{U} \in \mathcal{P}_{N}$ such that

$$
T_{V}(A)=\mathrm{VAV}^{-1} \text { and } T_{U}(A)=\mathrm{U} A \mathrm{U}^{-1} \quad \text { for any } \quad A \in \mathcal{P}_{N} .
$$

$\mathrm{V}$ and $\mathrm{U}$ are usually called shift and evolution operators, respectively. In the classical continuum limit $a \rightarrow 0, \tau \rightarrow 0$ they reproduce the momentum and the Hamiltonian (6.13): $\mathrm{V} \rightarrow e+\frac{i}{\hbar} a P, \mathrm{U} \rightarrow e+\frac{i}{\hbar} \tau H$.

Our interpretation of the transformations in Lemma 2 as discrete shifts in space and time, motivate to introduce the the objects $J_{n}^{\alpha}(t), g_{n}(t) \in \mathcal{G}_{a} \otimes \mathcal{P}_{N}$ such that

$$
\begin{gathered}
J_{n}^{r}(t+\tau)=J_{n-1}^{r}(t), \quad J_{n}^{l}(t+\tau)=J_{n+1}^{l}(t), \\
g_{n}(t+\tau)=\left(J_{n+1}^{l}(t)\right)^{-1} g_{n}(t)\left(J_{n}^{r}(t)\right)^{-1}, \quad g_{n+1}(t)=\left(J_{n+1}^{l}(t)\right)^{-1} g_{n}(t) J_{n+1}^{r}(t)
\end{gathered}
$$

and $J_{n}^{\alpha}(0), g_{n}(0)$ coincide with our usual generators $J_{n}^{\alpha}, g_{n}$, respectively. These expressions define $J_{n}^{\alpha}(t)$ and $g_{n}(t)$ for $t=k \tau$ with $k$ being integer. Below we shall also need the equations inverse to (6.18)- 6.19):

$$
\begin{aligned}
& J_{n}^{r}(t-\tau)=J_{n+1}^{r}(t), \quad J_{n}^{l}(t-\tau)=J_{n-1}^{l}(t), \\
& g_{n}(t-\tau)=J_{n}^{l}(t) g_{n}(t) J_{n+1}^{r}(t), \quad g_{n-1}(t)=J_{n}^{l}(t) g_{n}(t)\left(J_{n}^{r}(t)\right)^{-1} \text {. }
\end{aligned}
$$

We can now use the rules $J_{n}^{\alpha}(t) \rightarrow e \otimes \mathrm{e}-a j^{\alpha}(x, t)$ and $g_{n}(t) \rightarrow g(x, t)$ from Subsection 6.1 (here $x=n a=2 \pi n / N$ as before) to establish that in the classical continuum limit eqs. (6.18)- 6.19) become precisely the equations (6.11)-(6.12) 99. Further, combining (6.19) and (6.21), we obtain the following relations:

$$
\left(g_{n-1}(t)\right)^{-1} g_{n}(t-\tau)=J_{n}^{r} J_{n+1}^{r}, \quad g_{n-1}(t)\left(g_{n}(t+\tau)\right)^{-1}=J_{n+1}^{l} J_{n}^{l} .
$$

\footnotetext{
${ }^{18}$ This needs the following variant of eq. $(5.32): \mathcal{S}_{a}\left(\Phi_{n+1}^{l}\right)=\left(J_{n+1}^{l}\right)^{-1} \mathcal{S}_{a}\left(\Phi_{n}^{l}\right)$; it is evident if we take relation (3.19) into account.

${ }^{19}$ In the continuum limit the quotient $c:=a / \tau$ (speed of light) is supposed to be fixed. In fact, eq. (6.9) implies that we put $c=1$.
} 
These are lattice analogues of the definitions $(6.10)$ of the chiral currents. Notice that we can express only products of currents on neighboring links through the field $g_{n}(t)$ (nevertheless, in the classical continuum limit, eq. (6.10) is certainly recovered). 20

Eqs. (6.22) allow to obtain the dynamics of the lattice model in terms of the field $g_{n}$ only. Indeed, since their r.h.s. are manifestly chiral objects, the combinations of $g_{n^{-}}$ variables on the l.h.s. are to be invariant under the substitutions $t \rightarrow t+\tau, n \rightarrow n+1$ and $t \rightarrow t+\tau, n \rightarrow n-1$, respectively. Thus, we derive a lattice analogue of the equation of motion (6.9):

$$
g_{n+1}(t)\left(g_{n}(t-\tau)\right)^{-1}=g_{n}(t+\tau)\left(g_{n-1}(t)\right)^{-1} .
$$

Being a discrete analogue of an equation of second order in both variables, this relation involves four different points on the space-time lattice (see Figure 5). A natural choice of the initial data for eq. (6.23) is provided by the set $g_{n}(t-\tau)$ and $g_{n}(t), n=0, . ., N-1$ (here $t$ is fixed). It is interesting to notice that this set is divided into two subsets (black and white circles on Figure 5) which have an independent evolution; ${ }^{27}$ that is, the solution constructed according to eq. (6.23) from one of the sets never interacts with that constructed from the other set. According to eqs. (6.22), the initial data $g_{n}(t-\tau), g_{n}(t)$ (at fixed time $t$ ) can be restored if we are given the set of currents $J_{n}^{\alpha}, n=1, . ., N$ and two values of the $g$-field taken at two arbitrary points of the independent subsets, e.g. $g_{0}(0)$ and $g_{0}(-\tau)$. This is a lattice analogue of the initial data usually used in the continuum Hamiltonian approach (see above).
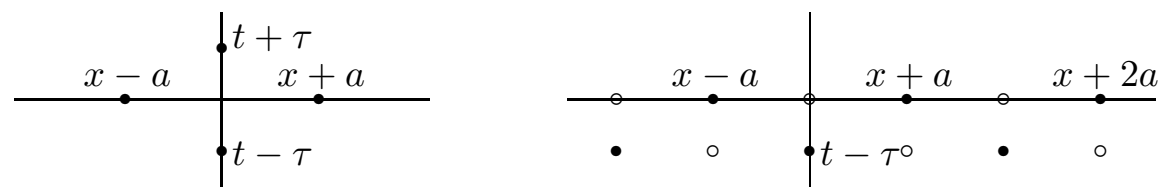

Figure 5: Graphical presentation of the discrete equation (6.23) and a possible choice of the initial data. The two subsets of the initial data have independent evolutions.

To summarize, in this section we have demonstrated that the elements $J_{n}^{\alpha}$ and $g_{n}$ which constitute the "physical" variables in the algebra $\mathcal{W}_{N}$ are indeed quantum lattice analogues of the chiral currents and the group valued field in the WZNW model.

6.4 $U(1)-W Z N W$ model. We conclude this section with some comments on the $\mathcal{Z}_{q^{-}}$ case. Recall that the $g$-field constructed from lattice vertex operators in the case of $\mathcal{G}=\mathcal{Z}_{q}$ is given by (cf. Subsection 5.5) $g_{n}=e^{\widehat{p} \otimes \phi_{n}}$, where $\phi_{n}=\widehat{\varsigma}_{n}^{r}-\widehat{\varsigma}_{n}^{l}$ is an operator acting on the physical space $\mathcal{H}_{N}$ (see Subsections 3.5 and 5.5). In the classical limit $\phi_{n}(t)$ becomes a lattice variable which, according to $(6.23)$, obeys to the following equation of motion:

$$
\phi_{n}(t+\tau)+\phi_{n}(t-\tau)=\phi_{n+1}(t)+\phi_{n-1}(t) .
$$

${ }^{20}$ Formally, we can split eqs. (6.22) into the following relations: $J_{n}^{r}=\left(g_{n-1}(t)\right)^{-1} g_{n-\frac{1}{2}}\left(t-\frac{1}{2} \tau\right)$ and $J_{n+1}^{r}=\left(g_{n-\frac{1}{2}}\left(t-\frac{1}{2} \tau\right)\right)^{-1} g_{n}(t-\tau)$. However, the variables assigned to half integer sites or times are not defined in the lattice formalism. To avoid this we could consider these relations as relations in $\mathcal{W}_{N}$ and re-express the involved $g$-fields through vertex operators, while using that vertex operators are chiral to replace formal variables on half integer space-time points by true objects of the lattice theory. As a result we would get the obvious relations $J_{n}^{\alpha}(t)=\left(\Phi_{n-1}^{\alpha}(t)\right)^{-1} \Phi_{n}^{\alpha}(t)$.

${ }^{21}$ Let us stress that it is not necessary to impose a continuity condition on the initial data, i.e., to demand that they possess smooth continuum limit. Moreover, it seems interesting to study the case when the two independent subsets of initial data have different continuum limits (cf. also [28]). 
This relation discretizes the equation of motion $\partial_{+} \partial_{-} \phi(x, t)=0$ of a free field. The latter is known to arise, in particular, for the continuum $U(1)$-WZNW model. Moreover, in the classical continuum limit the standard Poisson structure of the abelian WZNW theory is easily recovered from the exchange relations of our $\mathcal{Z}_{q}$ lattice model. These two observations allow to identify the $\mathcal{Z}_{q}$ lattice theory as a quantized lattice $U(1)$-WZNW model. In spite of its simplicity, the $U(1)$-theory has a lot of structure in common with the more complicated nonabelian models. In fact, the abelian model was used here to illustrate many elements of our general theory.

It is also worth mentioning that the abelian lattice theory itself has non-trivial mathematical aspects. In particular, explicit formulae for shift operators in chiral theories have been worked out in [31, 28, 8]. We may use these results to present expressions for the the shift and evolution operators $V$ and $U$. The latter can be decomposed into the chiral components: $V=V_{l} V_{r}$ and $U=V_{l} V_{r}^{-1}$. When acting on elements of the algebra $\mathcal{W}_{N}$, the operators $V_{\alpha} \in \mathcal{W}_{N}^{\alpha}$ generate shifts for the chiral sectors, i.e.,

$$
\left(e \otimes V_{\alpha}\right) J_{n}^{\alpha}\left(e \otimes V_{\alpha}\right)^{-1}=J_{n+1}^{\alpha}, \quad\left(e \otimes V_{\alpha}\right) \Phi_{n}^{\alpha}\left(e \otimes V_{\alpha}\right)^{-1}=\Phi_{n+1}^{\alpha},
$$

where $\alpha=r, l, n \in \mathbb{Z}$, and $e \otimes V_{l}, e \otimes V_{r}$ commute with any element from $\mathcal{G}_{a} \otimes \mathcal{W}_{N}^{r}$ and $\mathcal{G}_{a} \otimes \mathcal{W}_{N}^{l}$, respectively.

Proposition 6 (Shifts operators for $\mathcal{Z}_{q}$ ) Let $\mathcal{W}_{N}$ be the algebra of lattice vertex operators as defined in Subsection 5.5, i.e., it is generated by the elements $\widehat{W}_{n}^{\alpha}=e^{\widehat{\varpi}_{n}^{\alpha}}$ (chiral currents) and $\widehat{Q}_{n}^{\alpha}=e^{\widehat{\zeta}_{n}^{\alpha}}$ (vertex operators) obeying the relations spelled out in Subsections 4.4 and 5.5. Let the lattice length $N$ be odd. Then the chiral shift operators obeying 6.25) are given by

$$
V_{\alpha}=Z_{\alpha} \prod_{k=1}^{N-1} \rho_{\alpha}(N-k),
$$

where $\rho_{r}(k)=\exp \left\{-\frac{1}{2 \ln q}\left(\widehat{\varpi}_{k}^{r}\right)^{2}\right\}$ and $\rho_{l}(k)=\exp \left\{\frac{1}{2 \ln q}\left(\widehat{\varpi}_{k}^{l}\right)^{2}\right\}$. The function $Z_{\alpha}$ depends only on the element $C_{\alpha}=\left(\prod_{k=1}^{\frac{N+1}{2}} \widehat{W}_{2 k-1}^{\alpha}\right)\left(\prod_{k=1}^{\frac{N-1}{2}} \widehat{W}_{2 k}^{\alpha}\right)^{-1} \in \mathcal{K}_{N}^{\alpha}$.

To verify that $V_{r}$ and $V_{l}$ obey eqs. (6.25) one proceeds in two steps. The first of them concerns the relations between $V_{\alpha}$ and the chiral currents and it has been performed in [8]. The computation is based on the following relations: 2

$$
e^{\widehat{\varpi}_{n}^{\alpha}} \rho_{\alpha}(n+1) \rho_{\alpha}(n)=\rho_{\alpha}(n+1) \rho_{\alpha}(n) e^{\widehat{\varpi}_{n+1}^{\alpha}}, \quad n=1, . ., N-2,
$$

which hold due to eqs. (4.27). The remaining relations for $n=N-1, N$ are consequences of the others if the function $Z_{\alpha}$ is chosen in a specific way (see [8] for details).

In the second step, one checks the desired properties of $V_{\alpha}$ with respect to chiral vertex operators. To this end we derive the following relation:

$$
e^{\widehat{\varsigma}_{n}^{\alpha}} \rho_{\alpha}(n+1)=\rho_{\alpha}(n+1) e^{-\frac{1}{2} \ln q} e^{\widehat{\varpi}_{n+1}^{\alpha}} e^{\widehat{\varsigma}_{n}^{\alpha}}=\rho_{\alpha}(n+1) e^{\widehat{\varsigma}_{n}^{\alpha}+\widehat{\varpi}_{n+1}^{\alpha}}=\rho_{\alpha}(n+1) e^{\widehat{\varsigma}_{n+1}^{\alpha}} .
$$

For the first equality we used the commutation relations (5.45). After this, the CampbellHausdorff formula was employed before we could insert eqs. (5.44). Notice that it suffices to prove eq. (6.27) for $n=1$. Due to (5.44), the relations between $V_{\alpha}$ and the vertex

\footnotetext{
${ }^{22}$ Such relations were used first in $[31,28]$ in the construction of shift operators for $U(1)$-current algebra for even $N$ (cf. remarks in the text).
} 
operators assigned to other sites are consequences of this case (as soon as the relations for $V_{\alpha}$ with chiral currents are established). This completes the proof.

Let us comment on the construction of the chiral shift operators for a lattice of even length $N$ suggested in [31, 28]. In this case the shift operators are also given by (6.26) but without the factor $Z_{\alpha}$. When checking the relations between these operators $V_{\alpha}$ and chiral currents, Faddeev and Volkov had to assume that $\prod_{k=1}^{N / 2} \widehat{W}_{2 k-1}^{\alpha}=\prod_{k=1}^{N / 2} \widehat{W}_{2 k}^{\alpha}$. Unfortunately, such a constraint is incompatible with the exchange relations in the full theory which includes the objects $N_{n}$ in addition to chiral currents. One way to bypass this problem would involve shifts by two lattice units.

Let us finally mention that the function $\rho$ (which can be identified as a $\theta$-function, if written in terms of $\widehat{W}_{n}^{\alpha}$ ) appearing in (6.26) admits factorization into a product of two functions of a q-dilogarithm type (see [28]). Actually, these objects (the $\theta$-function and the q-dilogarithm) turn out to be quite universal building blocks for shift operators. They were employed in the recent work [32] to construct shift operators for the $S U(2)$-lattice KM algebra. Since the expressions involving $\theta$-functions and q-dilogarithms resemble those used in the abelian theory, one expects that the new operators of [32] serve as shifts not only for the current algebras but for the whole algebras of vertex operators as well.

\section{CONCLUSION}

In the present paper we have described the construction of lattice vertex operators for a given modular Hopf algebra. The investigation of the classical continuum limit reveals a clear relation between the lattice algebras and the WZNW-model. Since the latter can be reduced to the affine Toda model, our technique may be applied to this theory as well (with certain modifications). Furthermore, there exist many connections with Chern-Simons theory in $2+1$ dimensions (see []] for lattice constructions of Chern-Simons observables) which motivate to extend our framework to two spatial dimensions.

Let us briefly list some aspects of the presented theory which have not been developed. As we mentioned before, formulae for vertex operators are known only for some particular cases. It would be interesting to work out explicit presentations for universal vertex operators $\Phi$ of the deformed universal enveloping algebras $U_{q}(\mathfrak{G})$. Alternatively, one may try to find universal structure data $F, \sigma$ which solve the discussed set of equations. A further natural extension is to incorporate infinite dimensional structures such as the deformed affine algebras. This might allow for a comparison with the approaches in [34, 38] (see also references therein).

Another problem which is to be solved to complete the description of the quantum lattice WZNW model is an explicit construction of the shift and evolution operators. By now, exact formulae have been found for the cases of $U(1)$ and $S U(2)$ [31, 28, 8, 32]. These examples, however, hint at some uniform structures (such as the appearance of qdilogarithms) that might lead to new formulae for shift operators in more general theories.

Acknowledgments: We would like to thank A.Yu.Alekseev, L.D.Faddeev, A.Fring, J.Fröhlich, P.P.Kulish, F.Nill, A.Yu.Volkov for useful discussions and E.Jagunova for preparing the pictures. A.B. is grateful to Prof. R.Schrader for the hospitality at the Institut für Theoretische Physik, Freie Universität Berlin, and to the Töpfer Stiftung for financial support. 


\section{APPENDIX: SOME PROOFS AND FURTHER RELATIONS}

A.1 Proof of Proposition 1. It is not difficult to obtain the relations stated in Proposition 11 from the defining properties of $\Phi$. For instance, the formula (2.23) for $D$ follows from the definition (2.19) when $N=R R^{\prime}$ is re-expressed in terms of the ribbon element according to eq. (2.1). To derive equation (2.24) one needs no more than associativity of the multiplication in $\mathcal{V}$ along with co-associativity of the co-product $\Delta_{a}$ on $\mathcal{G}_{a}$,

$$
\begin{gathered}
\left.\stackrel{3}{\sigma}\left(F_{12}\right)\left(\Delta_{a} \otimes i d\right)(F)\left(\Delta_{a} \otimes i d\right) \Delta_{a}(\Phi)=\stackrel{3}{\sigma}\left(F_{12}\right) \stackrel{3}{\Phi} \Delta_{a}(\Phi)\right)_{12}=\stackrel{3}{\Phi}(\stackrel{2}{\Phi} \stackrel{1}{\Phi})= \\
=(\stackrel{2}{\Phi} \stackrel{2}{\Phi}) \stackrel{1}{\Phi}=F_{23} \Delta_{a}(\Phi)_{23} \quad \stackrel{1}{\Phi}=F_{23}\left(i d \otimes \Delta_{a}\right)(F)\left(i d \otimes \Delta_{a}\right) \Delta_{a}(\Phi) .
\end{gathered}
$$

The first relation of eqs. (2.25) is a consequence of the covariance property (2.16) of $\Phi$,

$$
\begin{gathered}
\stackrel{1}{D} \mathcal{R}_{-} \stackrel{2}{\Phi} \stackrel{1}{\Phi}=\stackrel{1}{\Phi} \stackrel{1}{N} \stackrel{1}{\Phi}{ }^{-1} \mathcal{R}_{-} \stackrel{2}{\Phi} \stackrel{1}{\Phi}=\stackrel{1}{\Phi} \stackrel{1}{N} \stackrel{2}{\Phi} R_{-}=\stackrel{1}{\Phi} \stackrel{2}{\Phi} R_{+} \stackrel{1}{N}= \\
=\mathcal{R}_{+} \stackrel{2}{\Phi} \stackrel{1}{\Phi} \stackrel{1}{N}=\mathcal{R}_{+} \stackrel{2}{\Phi} \stackrel{1}{D} \stackrel{1}{=}=\mathcal{R}_{+} \stackrel{2}{\sigma}(D) \stackrel{2}{\Phi} \stackrel{1}{\Phi} .
\end{gathered}
$$

We have inserted the definition (2.19) twice and used commutation relations (2.22).

Next, using definitions (2.17)-(2.18) of the structure data, we easily check (2.26):

$$
\begin{gathered}
\stackrel{2}{\sigma} \stackrel{1}{\sigma}(\mathrm{f})=\stackrel{2}{\Phi} \stackrel{1}{\Phi}(e \otimes \mathrm{f}) \stackrel{1}{\Phi}{ }^{-1} \stackrel{2}{\Phi}{ }^{-1}= \\
=F \Delta_{a}(\Phi)(e \otimes \mathrm{f}) \Delta_{a}\left(\Phi^{-1}\right) F^{-1}=F \Delta_{a}\left(\Phi(e \otimes \mathrm{f}) \Phi^{-1}\right) F^{-1}=\Delta_{F}(\sigma(\mathrm{f})) .
\end{gathered}
$$

Let us finally discuss the computation of $F^{*}$. It is based on the second identity in (2.11) and on the relation $(e \otimes S)(i d \otimes \Delta)(S)=(S \otimes e)(\Delta \otimes i d)(S)$ which can be checked in straightforward way. Applying them and the property (2.14) to the definition (2.17), we derive

$$
\begin{aligned}
F^{*} & =\left(\stackrel{2}{\Phi} \stackrel{1}{\Phi} \Delta_{a}\left(\Phi^{-1}\right)\right)^{*}=\Delta_{a}^{\prime}(\Phi S)\left(S^{-1}\right)_{13} \stackrel{1}{\Phi}^{-1}\left(S^{-1}\right)_{23} \stackrel{2}{\Phi^{-1}} \\
& =\Delta_{a}^{\prime}(\Phi)\left(\Delta^{\prime} \otimes i d\right)(S)\left[\left(e \otimes S^{-1}\right)\left(i d \otimes \Delta^{\prime}\right)\left(S^{-1}\right)\right]_{213} \stackrel{1}{\Phi^{-1}} \stackrel{2}{\Phi^{-1}} \\
& =\Delta_{a}^{\prime}(\Phi)\left(\Delta^{\prime} \otimes i d\right)(S)\left[(i d \otimes \Delta)\left(S^{-1}\right)\left(e \otimes S^{-1}\right)\right]_{213} \stackrel{1}{\Phi^{-1}} \stackrel{2}{\Phi}^{-1} \\
& =\Delta_{a}^{\prime}(\Phi)(S \otimes e) \stackrel{1}{\Phi^{-1}} \stackrel{2}{\Phi^{-1}}=S_{a} \Delta_{a}(\Phi) \stackrel{1}{\Phi^{-1}} \stackrel{2}{\Phi^{-1}}=S_{a} F^{-1}
\end{aligned}
$$

The index on $[.]_{213}$ refers to a permutation of tensor factors in the expression enclosed by the brackets.

All other relations in Proposition 1 are either obvious or they follow directly from the derived equations. This applies in particular to eq. (2.27).

A.2 Proof of Proposition 3. In this subsection we want to construct consistent structure data for vertex operators of the deformed universal enveloping algebras $\mathcal{G}=$ $U_{q}(\mathfrak{G})$ from their Clebsch-Gordan maps and $6 j$-symbols. To fix our notations, let us recall that the Clebsch-Gordan maps $C[T L \mid S]: V^{T} \otimes V^{L} \mapsto V^{S}$ have the following properties:

$$
\begin{aligned}
& C[T L \mid S]\left(\tau^{T} \otimes \tau^{L}\right)(\Delta(\xi))=\tau^{S}(\xi) C[T L \mid S] \quad \text { for all } \quad \xi \in \mathcal{G}, \\
&\left(\frac{\kappa_{S}}{\kappa_{T} \kappa_{L}}\right)^{ \pm 1} C[T L \mid S] R_{ \pm}^{T L} C[T L \mid R]^{*}=\delta_{R, S} \quad \text { with } \quad \kappa_{L}=\tau^{L}(\kappa)
\end{aligned}
$$




$$
\begin{aligned}
& \sum_{Q} B_{Q S}^{ \pm}\left[\begin{array}{ll}
L & T \\
R
\end{array}\right] C[J Q \mid R] C_{23}[T L \mid Q]=C[J S \mid R] C_{23}[T L \mid S]\left(R_{ \pm}^{J T} \otimes e^{L}\right) \\
& \sum_{Q} F_{Q S}\left[\begin{array}{ll}
L & T \\
R & J
\end{array}\right] C[Q L \mid R] C_{12}[J T \mid Q]=C[J S \mid R] C_{23}[T L \mid S]
\end{aligned}
$$

In the order of appearance here these equations describe the intertwining properties of the Clebsch-Gordan maps, their normalization and the definition of the braiding and fusion matrices (or $6 j$-symbols). It is well known that certain (polynomial) relations for the numbers $\left.B_{. .}^{ \pm .} \cdot\right]$ and $F_{\text {... }}[\cdot]$ follow from their definitions and properties of the quasitriangular Hopf algebra $\mathcal{G}$ (see, e.g., [39]). In particular, one has

$$
\begin{aligned}
\sum_{Q} F_{Q S}\left[\begin{array}{ll}
L & T \\
R & J
\end{array}\right] F_{N R}\left[\begin{array}{ll}
L & Q \\
P & I
\end{array}\right] F_{M Q}\left[\begin{array}{ll}
T & J \\
N & I
\end{array}\right] & =F_{M R}\left[\begin{array}{ll}
S & J \\
P & I
\end{array}\right] F_{N S}\left[\begin{array}{ll}
L & T \\
P & M
\end{array}\right], \\
\sum_{N} F_{N S}\left[\begin{array}{ll}
L & T \\
P & M
\end{array}\right] F_{N R}\left[\begin{array}{ll}
L & T \\
P & M
\end{array}\right]^{*} & =\delta_{S, R}, \\
\Omega_{+} \Omega_{-}^{-1}\left(\begin{array}{cc}
N_{P} & T
\end{array}\right) B_{N N^{\prime}}^{-}\left[\begin{array}{ll}
L & T \\
P
\end{array}\right] & =B_{N N^{\prime}}^{+}\left[\begin{array}{ll}
L & T \\
P
\end{array}\right] \Omega_{+} \Omega_{-}^{-1}\left(\begin{array}{cc}
{ }_{N} T \\
{ }_{N}
\end{array}\right) .
\end{aligned}
$$

Here we used the notation

$$
\Omega_{ \pm}\left(\begin{array}{cc}
L_{P} T \\
P
\end{array}\right)=B_{T L}^{ \pm}\left[\begin{array}{cc}
L_{P} & T
\end{array}\right] \text { and } \Omega_{+} \Omega_{-}^{-1}\left({ }_{P}^{L_{P}^{T}}\right)=\Omega_{+}\left({ }_{P}^{L_{P} T}\right) \Omega_{-}^{-1}\left({ }_{P}^{L_{P} T}\right)=\frac{v_{L} v_{T}}{v_{P}} .
$$

To proceed, we parameterize the labels $N, M, I$ and $N^{\prime}$ in terms of new variables $\lambda, \vartheta, \iota$ and $\vartheta^{\prime}$ so that

$$
N=P+\lambda, \quad M=P+\lambda+\vartheta, \quad I=P+\lambda+\vartheta+\iota, \quad N^{\prime}=P+\vartheta^{\prime} .
$$

Let us introduce the following matrices $\mathcal{C}\{T L \mid S\}(P), \mathcal{R}_{ \pm}^{T L}(P)$ and $D^{T}(P)$

$$
\begin{aligned}
\mathcal{C}\{T L \mid S\}(P)_{\varsigma, \vartheta \lambda} & =F_{P+\lambda S}\left[\begin{array}{l}
L \\
P
\end{array} \underset{P+\vartheta+\lambda}{T}\right] \delta_{\varsigma, \vartheta+\lambda}, \\
\mathcal{R}_{ \pm}^{T L}(P)_{\vartheta^{\prime} \lambda^{\prime}, \vartheta \lambda} & \left.=B_{P+\lambda P+\vartheta^{\prime}}^{ \pm}{ }_{P}^{L} \underset{P+\vartheta+\lambda}{T}\right] \delta_{\vartheta+\lambda, \vartheta^{\prime}+\lambda^{\prime}} \\
D^{T}(P)_{\vartheta^{\prime}, \vartheta} & =\Omega_{+} \Omega_{-}^{-1}\left({ }_{P+\vartheta}^{P}{ }_{P}^{T}\right) \delta_{\vartheta^{\prime}, \vartheta} .
\end{aligned}
$$

We may think of the $P$-dependent matrices $\mathcal{C}\{T L \mid S\}(P), \mathcal{R}_{ \pm}^{T L}(P)$ and $D^{T}(P)$ as matrices with entries in the algebra $\mathcal{C}$. Whenever we do so, we will neglect to write the $P$-dependence explicitly and use the symbols $\mathcal{C}\{T L \mid S\}, \mathcal{R}_{ \pm}^{T L}$ and $D^{T}$. If we introduce in addition the matrix valued map $\sigma^{L}$ on $\mathcal{C}$ by

$$
\sigma^{L}(P)_{\lambda^{\prime}, \lambda}=(P+\lambda) \delta_{\lambda^{\prime}, \lambda}
$$

then eqs. (A.2) for the fusion and braiding matrices become

$$
\begin{aligned}
\sum_{Q} F_{Q S}\left[\begin{array}{ll}
L & T \\
R
\end{array}\right] \mathcal{C}\{Q L \mid R\} \sigma^{L}\left(\mathcal{C}_{12}\{J T \mid Q\}\right) & =\mathcal{C}\{J S \mid R\} \mathcal{C}_{23}\{T L \mid S\} \\
\mathcal{C}\{T L \mid S\} \mathcal{C}\{T L \mid R\}^{*} & =\delta_{R, S} \\
\left(D^{T} \otimes e^{L}\right) \mathcal{R}_{-}^{T L} & =\mathcal{R}_{+}^{T L} \sigma^{L}\left(D^{T}\right) .
\end{aligned}
$$


The last of these equations appears already as a close relative of eq. 2.25). In fact, for semi-simple $\mathcal{G}$ one can construct universal objects $\mathcal{R}_{ \pm} \in \mathcal{G} \otimes \mathcal{G} \otimes \mathcal{C}$ and $D \in \mathcal{G} \otimes \mathcal{C}$ so that $\mathcal{R}_{ \pm}^{T L}=\left(\tau^{T} \otimes \tau^{L}\right)\left(\mathcal{R}_{ \pm}\right)$and $D^{T}=\tau^{T}(D)$. Then eq. (A.5) turns into

$$
\stackrel{1}{D} \mathcal{R}_{-}=\mathcal{R}_{+} \stackrel{2}{\sigma}(D)
$$

where $\sigma: \mathcal{C} \mapsto \mathcal{G} \otimes \mathcal{C}$ is defined so that $\sigma^{L}=\left(\tau^{L} \otimes i d\right) \circ \sigma$. When $\Omega_{+} \Omega_{-}^{-1}$ is expressed in terms of the ribbon element $v$ as in eq. A.3), the definition (A.4) of $D$ becomes

$$
D=\sigma(\mathrm{v}) \mathrm{v}^{-1} v_{a} .
$$

To build the universal element $F \in \mathcal{G} \otimes \mathcal{G} \otimes \mathcal{C}$, we combine the matrices $\mathcal{C}\{. . \mid$. $\}$ with the Clebsch-Gordan maps so that $F^{T L}=\left(\tau^{T} \otimes \tau^{L}\right)(F)$ is given by

$$
F^{T L} \equiv \sum_{S} \mathcal{C}\{T L \mid S\}^{*} C[T L \mid S]
$$

Multiplying the adjoint of the first eq. in (A.2) with eq. (A.1), taking sum over $R, S$ and using the intertwining properties of the Clebsch-Gordan maps, we obtain the equation (2.24) for $F$. In the same way, one may combine the normalizations for the ClebschGordan maps $C[T L \mid S]$ and the matrices $\mathcal{C}\{T L \mid S\}$ to derive that

$$
F\left(\left(\Delta\left(\kappa^{-1}\right)(\kappa \otimes \kappa) R_{+}^{-1}\right) \otimes \mathrm{e}\right) F^{*}=e \otimes e \otimes \mathrm{e},
$$

and hence $F$ has the required property under the $*$-operation. Finally, we use that the matrix $\mathcal{C}\{T L \mid S\}(P)$ is proportional to $\delta_{\varsigma, \vartheta+\lambda}$ so that

$$
(\mathrm{f}(P+\varsigma)) \mathcal{C}\{T L \mid S\}(P)_{\varsigma, \vartheta \lambda}=\mathcal{C}\{T L \mid S\}(P)_{\varsigma, \vartheta \lambda}(\mathrm{f}(P+\vartheta+\lambda)) .
$$

Here $f(P)$ is an arbitrary function of $P$, i.e., $f$ may be regarded as an element in $\mathcal{C}$. With our standard conventions, this can be stated as a matrix equation

$$
\sigma^{S}(\mathrm{f}) \mathcal{C}\{T L \mid S\}=\mathcal{C}\{T L \mid S\} \sigma^{L} \sigma^{T}(\mathrm{f}) .
$$

Keeping in mind that $\Delta_{F}(\xi)=F(\Delta(\xi) \otimes \mathrm{e}) F^{-1}$, we discover eq. (2.26). All other properties of the structure data follow easily from the relations we have discussed here.

A.3 Structure data for left chiral vertex operators. We can obtain the relations for the left structure data $F_{l}, \sigma_{l}, D_{l}, \mathcal{R}_{ \pm}^{l}$ from eqs. (2.23)-(2.28) if we substitute

$$
\begin{aligned}
& \Delta_{a} \rightarrow \Delta_{a}^{\prime} \quad F \rightarrow F_{l}^{\prime} \quad M \quad \rightarrow \quad\left(M^{l}\right)^{-1} \\
& R_{ \pm} \rightarrow R_{ \pm}^{\prime} \quad \sigma \rightarrow \sigma_{l} \quad \mathcal{R}_{ \pm} \rightarrow\left(\mathcal{R}_{ \pm}^{l}\right)^{\prime} \\
& v_{a} \rightarrow v_{a} \quad D \rightarrow D_{l}^{-1} \quad \Phi \quad \rightarrow \quad \Phi^{l} .
\end{aligned}
$$

The prime on $F_{l}, \mathcal{R}_{ \pm}^{l} \in \mathcal{G}_{a} \otimes \mathcal{G}_{a} \otimes \mathcal{C}^{l}$ denotes permutation of the first two tensor factors. Once the validity of these rules has been checked for the defining relations (2.17)-(2.19) of structure data, we can apply them to eqs. (2.23)-(2.28). Within the notations of Proposition 1, the result looks as follows:

$$
\begin{array}{r}
D_{l}=\left(v_{a}^{-1} \mathrm{v}\right) \sigma_{l}\left(\mathrm{v}^{-1}\right) \quad, \quad \stackrel{1}{\sigma}_{l} \stackrel{2}{\sigma}_{l}\left(\mathrm{f}_{l}\right)=\Delta_{F_{l}}\left(\sigma_{l}\left(\mathrm{f}_{l}\right)\right), \\
{\left[F_{l} \otimes e\right]_{1243}\left(\left(\Delta_{a} \otimes i d\right)\left(F_{l}\right)\right)=\stackrel{1}{\sigma}\left(F_{l}\right)\left(\left(i d \otimes \Delta_{a}\right)\left(F_{l}\right)\right),}
\end{array}
$$




$$
\begin{aligned}
& \stackrel{2}{D_{l}} \mathcal{R}_{+}^{l}=\mathcal{R}_{-}^{l} \stackrel{1}{\sigma_{l}}\left(D_{l}\right) \quad, \quad \mathcal{R}_{+}^{l} \stackrel{1}{D_{l}}=\stackrel{2}{\sigma_{l}}\left(D_{l}\right) \mathcal{R}_{-}^{l}, \\
& \mathcal{R}_{ \pm, 23}^{l} \stackrel{2}{\sigma}_{l}\left(\mathcal{R}_{ \pm, 13}^{l}\right) \mathcal{R}_{ \pm, 12}^{l}=\stackrel{3}{\sigma}_{l}\left(\mathcal{R}_{ \pm, 12}^{l}\right) \mathcal{R}_{ \pm, 13}^{l} \stackrel{1}{\sigma}_{l}\left(\mathcal{R}_{ \pm, 23}^{l}\right), \\
& F_{l}^{*}=S_{a}^{-1} F_{l}^{-1} \quad, \quad\left(\mathcal{R}_{ \pm}^{l}\right)^{*}=\left(\mathcal{R}_{ \pm}^{l}\right)^{-1} \quad, \quad D_{l}^{*}=D_{l}^{-1}, \\
& \sigma_{l}\left(\mathrm{f}_{l}\right)^{*}=\sigma_{l}\left(\mathrm{f}_{l}^{*}\right) \quad, \quad \Delta_{F_{l}}(\xi)^{*}=\Delta_{F_{l}}\left(\xi^{*}\right) .
\end{aligned}
$$

Here $\Delta_{F_{l}}(\xi) \equiv F_{l}(\Delta(\xi) \otimes \mathrm{e}) F_{l}^{-1} \in \mathcal{G}_{a} \otimes \mathcal{G}_{a} \otimes \mathcal{V}_{l}$ analogously to definition (2.21). Using the fact that $\left(\mathcal{S}_{a} \otimes \mathcal{S}_{a}\right)\left(\Delta_{a}(\xi)\right)=\Delta_{a}^{\prime}\left(\mathcal{S}_{a}(\xi)\right)$, it is simple to see that the objects (3.13), (3.14) satisfy the relations (A.6)-(A.11) if $F, \sigma, \Delta, \mathcal{R}$ solve eqs. (2.23)-(2.28). The former equations are actually obtained from the latter by acting with the maps $\mathcal{S}_{l r}^{(n)}$ defined in eq. (3.15).

A.4 Properties of the field $g$. The consistency of the object $g$ with the constraint to the diagonal subspace $\mathcal{H}$ is certainly its most important property. It was formulated more precisely in (3.17) using notations from Subsection 3.4. A formal proof may be given as follows. Suppose that $\mathrm{f} g=\mathcal{S}_{l r}(\mathrm{f}) g$ holds for all $\mathrm{f} \in \mathcal{C}^{r}$. Then one finds for arbitrary $\mathrm{f} \in \mathcal{C}^{r}$

$$
\begin{aligned}
g \mathrm{f} & =\mathcal{S}_{a}\left(\Phi^{l}\right) \Phi^{r} \mathrm{f}=\mathcal{S}_{a}\left(\Phi^{l}\right) \sigma_{r}(\mathrm{f}) \Phi^{r}=\mathcal{S}_{a}\left(\left(\mathcal{S}^{-1} \otimes i d\right)\left(\sigma_{r}(\mathrm{f})\right) \Phi^{l}\right) \Phi^{r} \\
& =\mathcal{S}_{a}\left(\left(\mathcal{S}^{-1} \otimes \mathcal{S}_{l r}\right)\left(\sigma_{r}(\mathrm{f})\right) \Phi^{l}\right) \Phi^{r}=\mathcal{S}_{a}\left(\sigma_{l}\left(\mathcal{S}_{l r}(\mathrm{f})\right) \Phi^{l}\right) \Phi^{r} \\
& =\mathcal{S}_{a}\left(\Phi^{l}\right) \mathcal{S}_{l r}(\mathrm{f}) \Phi^{r}=\mathcal{S}_{a}\left(\Phi^{l}\right) \Phi^{r} \mathcal{S}_{l r}(\mathbf{f})=g \mathcal{S}_{l r}(\mathrm{f})
\end{aligned}
$$

The computation makes use of the choice (3.14) to replace $\sigma_{r}$ by $\sigma_{l}$. From now on, we think of $g$ as being restricted to $\mathcal{H}$. We begin our proof of Proposition 4 with the operator product expansion $(3.20)$ of $g \in \mathcal{G}_{a} \otimes \operatorname{End}(\mathcal{H})$,

$$
\begin{aligned}
\stackrel{2}{g} \stackrel{1}{g} & =\mathcal{S}_{a}\left(\stackrel{2}{\Phi^{l}}\right) \mathcal{S}_{a}\left(\stackrel{1}{\Phi^{l}}\right) \stackrel{2}{\Phi^{r}} \stackrel{1}{\Phi^{r}}=(\mathcal{S} \otimes \mathcal{S} \otimes i d)\left(F_{l}^{\prime} \Delta_{a}^{\prime}\left(\Phi^{l}\right)\right) F_{r} \Delta_{a}\left(\Phi^{r}\right) \\
& =(\mathcal{S} \otimes \mathcal{S} \otimes i d)\left(\left(\mathcal{S}^{-1} \otimes \mathcal{S}^{-1} \otimes i d\right)\left(F_{r}^{-1}\right) \Delta_{a}^{\prime}\left(\Phi^{l}\right)\right) F_{r} \Delta_{a}\left(\Phi^{r}\right) \\
& =\Delta_{a}\left(\mathcal{S}_{a}\left(\Phi^{l}\right)\right) F_{r}^{-1} F_{r} \Delta_{a}\left(\Phi^{r}\right)=\Delta_{a}(g) .
\end{aligned}
$$

Here $\mathcal{S}_{a}(\stackrel{1}{\Phi})$ and $\mathcal{S}_{a}(\stackrel{2}{\Phi})$ are shorthands for $\left(\mathcal{S}_{a} \otimes i d\right)(\stackrel{1}{\Phi})$ and $\left(i d \otimes \mathcal{S}_{a}\right)(\stackrel{2}{\Phi})$, respectively. The exchange relations for $g$, the formula $g^{-1}=\mathcal{S}_{a}(g)$ and the normalization $\epsilon_{a}(g)=\mathrm{e}$ follow immediately from the functoriality relation in (3.20).

The exchange relations (3.21) are derived from (3.3), (3.8) and the explicit construction of $g$ as a product of $\mathcal{S}_{a}\left(\Phi^{l}\right)$ and $\Phi^{r}$. Let us check the first of them:

$$
\stackrel{1}{M}^{r} \stackrel{2}{g} R_{-}=\stackrel{1}{M}^{r} \mathcal{S}_{a}\left(\stackrel{2}{\Phi}{ }^{l}\right) \stackrel{2}{\Phi} R^{r} R_{-}=\mathcal{S}_{a}\left(\stackrel{2}{\Phi}{ }^{l}\right) \stackrel{1}{M^{r}} \stackrel{2}{{ }^{r}} R_{-}=\mathcal{S}_{a}(\stackrel{2}{\Phi} l) \stackrel{2}{\Phi}{ }^{r} R_{+} \stackrel{1}{M^{r}}=\stackrel{2}{g} R_{+} \stackrel{1}{M}^{r}
$$

The second relation in (3.21) can be obtained similarly if we take the covariance properties (3.18) of $\mathcal{S}_{a}\left(\Phi^{l}\right)$ into account.

Verification of the relations (3.22) makes use of the equality $\mathrm{v}_{l}=\mathrm{v}_{r}$ which is valid on $\mathcal{H}$ and follows with the help of $\mathcal{S}(v)=v$, if the constraint $\mathcal{S}_{l r}\left(\mathrm{f}_{r}\right)=\mathrm{f}_{l}$ is evaluated on the ribbon element. The second relation in (3.22) then is obvious, and for the first we check explicitly:

$$
g_{n} M_{n}^{r}=g_{n}\left(\Phi_{n}^{r}\right)^{-1} v_{a}^{-1} D_{r} \Phi_{n}^{r}=\mathcal{S}_{a}\left(\Phi_{n}^{l}\right) v_{a}^{-1} D_{r} \Phi_{n}^{r}
$$




$$
\begin{aligned}
& =\left(\Phi_{n}^{l}\right)^{-1} \theta_{l} \mathrm{v}_{r}^{-1} \sigma_{r}\left(\mathrm{v}_{r}\right) \Phi_{n}^{r}=\mathrm{v}_{l}^{-1}\left(\Phi_{n}^{l}\right)^{-1} \theta_{l} \Phi_{n}^{r} \mathrm{v}_{l}=\left(\Phi_{n}^{l}\right)^{-1} \sigma_{l}\left(\mathrm{v}_{l}^{-1}\right) \mathrm{v}_{l} \theta_{l} \Phi_{n}^{r} \\
& =\left(\Phi_{n}^{l}\right)^{-1} v_{a} D_{l} \Phi_{n}^{l}\left(\Phi_{n}^{l}\right)^{-1} \theta_{l} \Phi_{n}^{r}=M_{n}^{l} \mathcal{S}_{a}\left(\Phi_{n}^{l}\right) \Phi_{n}^{r}=M_{n}^{l} g_{n} .
\end{aligned}
$$

In this computation it was convenient to insert the formula (3.19) which expresses $\mathcal{S}_{a}\left(\Phi^{l}\right)$ in terms of $\left(\Phi^{l}\right)^{-1}$.

A.5 Properties of lattice vertex operators. Let us prove that the structure constants $F_{\alpha}, \mathcal{R}_{ \pm}^{\alpha}$ and $\sigma_{\alpha}$ appearing in eqs. (5.20)-(5.22) coincide with those of the vertex operators $\Phi_{0}^{\alpha}$. To this end, we exploit the construction of $\Phi_{n}^{\alpha}$ as a product of $\Phi_{0}^{\alpha}$ and the holonomies $U_{n}^{\alpha}$ (see eq. (5.16)). Equation (5.22) is actually obvious, since $\mathrm{f}_{\alpha}$ commute with all the elements $U_{n}^{\alpha}$. Furthermore, eq. (5.21) is a simple consequence of (5.20). Hence, we need to prove only eq. (5.20) which we do for the right chirality ( the left one works analogously),

$$
\begin{aligned}
\Delta_{a}\left(\Phi_{n}^{r}\right) & =\Delta_{a}\left(\Phi_{0}^{r}\right) \Delta_{a}\left(U_{n}^{r}\right)=F_{r}^{-1} \stackrel{2}{\Phi_{0}^{r}} \stackrel{1}{\Phi}_{0}^{r} R_{-}^{-1} \stackrel{2}{U_{n}^{r}} \stackrel{1}{U_{n}^{r}}= \\
& =F_{r}^{-1} \stackrel{2}{\Phi_{0}^{r}} \stackrel{2}{U}_{n}^{r} \stackrel{1}{\Phi_{0}^{r}} \stackrel{1}{U}_{n}^{r}=F_{r}^{-1} \stackrel{2}{\Phi}_{n}^{r} \stackrel{1}{\Phi}_{n}^{r} .
\end{aligned}
$$

The exchange relations (5.25)-(5.28) are established by induction. Indeed, for $n=0$ they are part of Definition 3 . Assume now that eqs. (5.25)-(5.28) hold for a certain $n<N$ so that, in particular, $\Phi_{n}^{\alpha}$ has non-trivial exchange relations with $J_{n}^{\alpha}$ and $J_{n+1}^{\alpha}$ only. Then $\Phi_{n+1}^{\alpha}=\Phi_{n}^{\alpha} J_{n+1}^{\alpha}$ necessarily commutes with all currents $J_{m}^{\alpha}$ except from $J_{n}^{\alpha}, J_{n+1}^{\alpha}$ and $J_{n+2}^{\alpha}$. It is easy to verify that the exchange relations with $J_{n}^{\alpha}$ become trivial as well. We demonstrate this for $\alpha=r$ :

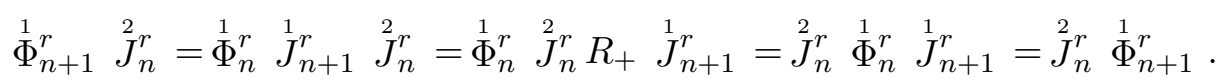

It can be checked similarly that the relations between $\Phi_{n+1}^{\alpha}$ and $J_{n+1}^{\alpha}, J_{n+2}^{\alpha}$ coincide with those between $\Phi_{n}^{\alpha}$ and $J_{n}^{\alpha}, J_{n+1}^{\alpha}$. This completes the induction.

Now we have to prove eqs. (5.30)-(5.31). For instance, using (4.17) and (5.25), we derive the first relation in (5.30) for $0 \leq n<m<N$ :

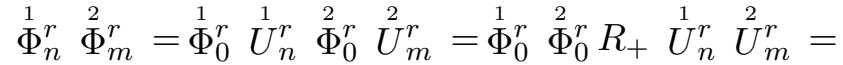

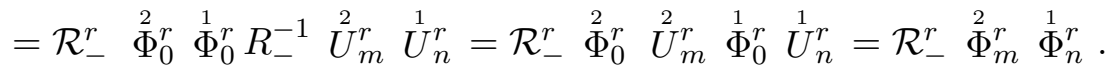

The relations (5.18)-(5.22), (5.24)-(5.28) which involve vertex operators $\Phi_{n}^{\alpha}$ outside of the initial interval $n=0, . ., N-1$, are derived with the help of eqs. (5.34)-(5.35) for the monodromies $M_{n}^{\alpha}$. Since the derivation uses the same technique as above, we prove only the functoriality relation for $\Phi_{n}^{r}$. As a first step, we check the following:

$$
\begin{gathered}
\Delta_{a}\left(\Phi_{n+N}^{r}\right)=\Delta_{a}\left(\Phi_{n}^{r}\right) \Delta_{a}\left(M_{n}^{r}\right)=F_{r}^{-1} \stackrel{2}{\Phi_{n}^{r}} \stackrel{1}{\Phi_{n}^{r}} R_{-}^{-1} \stackrel{2}{M_{n}^{r}} R_{+} \stackrel{1}{M}_{n}^{r}= \\
=F_{r}^{-1} \stackrel{2}{\Phi_{n}^{r}} \stackrel{2}{M_{n}^{r}} \stackrel{1}{\Phi_{n}^{r}} \stackrel{1}{M}_{n}^{r}=F_{r}^{-1} \stackrel{2}{\Phi_{n+N}^{r}} \stackrel{1}{\Phi}{ }_{n+N}^{r} .
\end{gathered}
$$

Then we use an induction and eq. (5.35) to get the same property for $\Phi_{n+k N}^{r}$.

Finally, we establish relations (5.36) directly with the help of eq. (5.35):

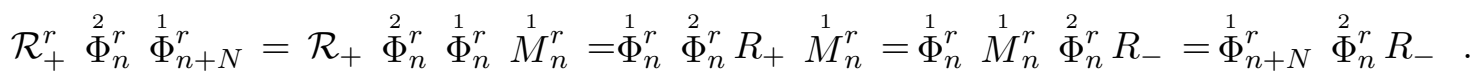

Detailed computations for the other relations in $(5.18)-(5.22),(5.24)-(5.28)$ can be worked out easily. 
A.6 Properties of lattice field $g_{n}$. Let us notice that the equality (3.19) holds in the lattice case for all $\Phi_{n}^{l}$ with the same $\theta_{l} \in \mathcal{G}_{a} \otimes \mathcal{C}^{l}$ (as we explained in Subsection 5.2, vertex operators of the same chirality assigned to different sites possess the same structure data). Therefore, we can proceed as in the toy model case and rewrite the expression for $g_{n}$ as follows:

$$
g_{n}=\left(\Phi_{n}^{l}\right)^{-1} \theta_{l} \Phi_{n}^{r}
$$

This relation allows to express $g_{n}$ in terms of $g_{0}$ and the holonomies $U_{n}^{\alpha} \in \mathcal{G}_{a} \otimes \mathcal{K}_{N}$ :

$$
g_{n}=\left(U_{n}^{l}\right)^{-1} g_{0} U_{n}^{r} .
$$

Bearing in mind that elements from $\mathcal{K}_{N}$, and hence, in particular, components of the holonomies $U_{n}^{\alpha}$, leave the subspaces $W_{N}^{\bar{K} K}$ of the full representation space $\mathcal{M}_{N}=\bigoplus_{I J} W_{N}^{I J}$ invariant (see Subsection 5.4), the equality (A.12) explains why all $g_{n}$ can be restricted on the diagonal subspace $\mathcal{H}_{N}=\bigoplus_{K} W_{N}^{\bar{K} K}$ simultaneously.

Among the properties of the lattice field $g_{n}$ in Proposition 5 , only the relations 5.42 and (5.40) have not been considered in the toy model case. Eqs. (5.42) follow immediately from the covariance properties (5.19) of the vertex operators and the remark that, due to eq. (3.19), the second relation in (5.19) can be rewritten as follows:

$$
\mathcal{S}_{a}\left(\Phi_{n}^{l}\right) \iota_{n}(\xi)=\Delta_{n}(\xi) \mathcal{S}_{a}\left(\Phi_{n}^{l}\right), \quad \mathcal{S}_{a}\left(\Phi_{n}^{l}\right) \iota_{m}(\xi)=\iota_{m}(\xi) \mathcal{S}_{a}\left(\Phi_{n}^{l}\right) \text { for } m \neq n(\bmod N)
$$

for all $\xi \in \mathcal{G}$.

The periodicity of $g_{n}$ is derived with the help of relations (5.15), (5.24) and the second equation in (3.22):

$$
\begin{aligned}
g_{n+N} & =\mathcal{S}_{a}\left(\Phi_{n+N}^{l}\right) \Phi_{n+N}^{r}=\left(\Phi_{n+N}^{l}\right)^{-1} \theta_{l} \Phi_{n+N}^{r}=\left(\Phi_{n}^{l} M_{n}^{l}\right)^{-1} \theta_{l} \Phi_{n}^{r} M_{n}^{r} \\
& =\left(\Phi_{n}^{l}\right)^{-1}\left(v_{a} D_{l}\right)^{-1} \theta_{l} v_{a}^{-1} D_{r} \Phi_{n}^{r}=\left(\Phi_{n}^{l}\right)^{-1} \sigma_{l}\left(\mathrm{v}_{l}\right) \mathrm{v}_{l}^{-1} \theta_{l} \sigma_{r}\left(\mathrm{v}_{r}\right) \mathrm{v}_{r}^{-1} \Phi_{n}^{r} \\
& =\mathrm{v}_{l}\left(\Phi_{n}^{l}\right)^{-1} \mathrm{v}_{l}^{-1} \theta_{l} \mathrm{v}_{r}^{-1} \Phi_{n}^{r} \mathrm{v}_{r}=\mathrm{v}_{r}\left(\Phi_{n}^{l}\right)^{-1} \mathrm{v}_{r}^{-1} \theta_{l} \mathrm{v}_{l}^{-1} \Phi_{n}^{r} \mathrm{v}_{l}=\mathcal{S}_{a}\left(\Phi_{n}^{l}\right) \Phi_{n}^{r}=g_{n} .
\end{aligned}
$$

Due to periodicity, it is sufficient to check the locality of $g_{n}$ only for $0 \leq n, m<N$. Taking, for definiteness, $n<m$, we derive:

$$
\begin{aligned}
& \stackrel{1}{g}_{n} \stackrel{2}{g}_{m}=\left(\stackrel{1}{U_{n}^{l}}\right)^{-1} \stackrel{1}{g}_{0} \stackrel{1}{U}_{n}^{r}\left(\stackrel{2}{U_{m}^{l}}\right)^{-1} \stackrel{2}{g}_{0} \stackrel{2}{U_{m}^{r}}=\left(\stackrel{1}{U_{n}^{l}}\right)^{-1}\left(\stackrel{2}{U_{m}^{l}}\right)^{-1} R_{-}^{-1} \stackrel{1}{g_{0}} \stackrel{2}{g}_{0} R_{+} \stackrel{1}{U_{n}^{r}} \stackrel{2}{{ }^{r}}{ }_{m}^{r}= \\
& =\left(\stackrel{2}{U_{m}^{l}}\right)^{-1}\left(\stackrel{1}{U^{l}}\right)^{-1} R_{+} \stackrel{2}{g}_{0} \stackrel{1}{g}_{0} R_{-}^{-1} \stackrel{2}{U_{m}^{r}} \stackrel{1}{U}_{n}^{r}=\left(\stackrel{2}{U_{m}^{l}}\right)^{-1} \stackrel{2}{g}_{0}\left(\stackrel{1}{U_{n}^{l}}\right)^{-1} \stackrel{2}{U}_{m}^{r} \stackrel{1}{g} \stackrel{1}{U}_{n}^{r}=\stackrel{2}{g} \stackrel{1}{g}_{n} \text {. }
\end{aligned}
$$

Here we used eq. (A.12) and the commutation relations between $g_{0}$ and the holonomies $U_{n}^{\alpha}$ which are obvious consequences of eqs. (5.43).

\section{References}

[1] Abe E.: Hopf algebras. Cambridge: Cambridge University Press 1980.

[2] Alekseev A.Yu., Faddeev L.D., Commun. Math. Phys. 141, 413 (1991).

[3] Alekseev A.Yu., Faddeev L.D.: An involution and dynamics for the q-deformed quantum top, preprint hep-th/9406196 (1994).

[4] Alekseev A.Yu., Faddeev L.D., Semenov-Tian-Shansky M.A., Commun. Math. Phys. 149, 335 (1992).

[5] Alekseev A.Yu., Faddeev L.D., Semenov-Tian-Shansky M.A., Volkov A.Yu.: The unraveling of the quantum group structure in the WZNW theory, preprint CERN-TH-5981/91 (1991).

[6] Alekseev A.Yu., Faddeev L.D., Fröhlich J., Schomerus V.: Representation theory of lattice current algebras, preprint q-alg/9604017 (1996), to appear in Commun. Math. Phys. 
[7] Alekseev A.Yu., Grosse H., Schomerus V., Commun. Math. Phys. 172, 317 (1995).

[8] Alekseev A.Yu., Recknagel A., Lett. Math. Phys. 37, 15 (1996).

[9] Alekseev A.Yu., Schomerus V., Duke Math. Journal 85, 457 (1996).

[10] Alekseev A.Yu., Shatashvili S.L., Commun. Math. Phys. 133, 353 (1990).

[11] Babelon O., Commun. Math. Phys. 139, 619 (1991).

[12] Babelon O., Bernard D., Billey E., Phys. Lett. B 375, 89 (1996).

[13] Balog J., Dabrowski L., Feher L., Phys. Lett. B 244, 227 (1990).

[14] Belavin A.A., Polyakov A.M., Zamolodchikov A.B., Nucl. Phys. B 241, 333 (1984).

[15] Biedenharn L.C., Lohe M.A.: Quantum group symmetry and q-tensor algebras. World Scientific 1995.

[16] Blok B., Phys. Lett. 233, 359 (1989).

[17] Bytsko A.G.: Tensor operators in R-matrix approach, preprint q-alg/9512025 (1995).

[18] Bytsko A.G.: Fusion of q-tensor operators: quasi-Hopf-algebraic point of view, preprint q-alg/9609007 (1996).

[19] Bytsko A.G., Faddeev L.D., J. Math. Phys. 37, 6324 (1996).

[20] Chu M., Goddard P., Halliday I., Olive D., Schwimmer A., Phys. Lett. B 266, 71 (1991).

[21] Chu M., Goddard P., Nucl. Phys. B 445, 145 (1995).

[22] Chu M., Goddard P., Phys. Lett. B 337, 285 (1994).

[23] Cremmer E., Gervais J.-L., Commun. Math. Phys. 134, 619 (1990).

[24] Drinfeld V.G. Quantum groups. In: Proc. of ICM, Berkeley 1986. AMS 1987; Sov. Math. Dokl. 32, 254 (1985).

[25] Drinfeld V.G., Algebra and Analysis 1, 1419 (1990).

[26] Faddeev L.D., Commun. Math. Phys. 132, 131 (1990).

[27] Faddeev L.D. Quantum symmetry in conformal field theory by Hamiltonian methods. In: New symmetry principles in quantum field theories. New York: Plenum Press 1992.

[28] Faddeev L.D.: Current-like variables in massive and massless integrable models, preprint hep-th/9408041 (1994).

[29] Faddeev L.D., Takhtajan L.A., Lect. Notes in Phys. 246, 166 (1986).

[30] Faddeev L.D., Reshetikhin N.Yu., Takhtajan L.A., Algebra and analysis 1, 193 (1990).

[31] Faddeev L.D., Volkov A.Yu., Phys. Lett. B 315, 311 (1993).

[32] Faddeev L.D., Volkov A.Yu.: Shift operators for non-abelian lattice current algebra, preprint hep-th/9606088 (1996).

[33] Falceto F., Gawedzki K., J. Geom. Phys. 11, 251 (1993).

[34] Frenkel I.B., Reshetikhin N.Yu., Commun. Math. Phys. 146, 1 (1992).

[35] Furlan P., Hadjiivanov L.K., Todorov I.T., Nucl. Phys. 474, 497 (1996).

[36] Gawedzki K., Commun. Math. Phys. 139, 201 (1990).

[37] Gervais J.-L., Neveu A., Nucl. Phys. B 238, 125 (1984).

[38] Jimbo M., Miwa T.: Algebraic analysis of solvable lattice models. CBMS Regional Conference Series in Mathematics, v. 85. AMS 1994.

[39] Kirillov A.N., Reshetikhin N.Yu., Adv. Series in Math. Phys. 11, 202. World Scientific 1990.

[40] Kirillov A.N., Reshetikhin N.Yu., Commun. Math. Phys. 134, 421 (1991).

[41] Knizhnik V.G., Zamolodchikov A.B., Nucl. Phys. B 247, 83 (1984).

[42] Kanie Y., Tsuchiya A., Adv. Studies in Pure Math. 16, 297 (1988).

[43] Mack G., Schomerus V., Nucl. Phys. B 370, 185 (1992).

[44] Moore G., Seiberg N., Commun. Math. Phys. 123, 177 (1989).

[45] Nill F., Szlachanyi K.: Quantum chains of Hopf algebras with order-disorder fields and quantum double symmetry, preprint hep-th/9507174 (1995); Quantum chains of Hopf algebras with quantum double cosymmetry, preprint hep-th/9509100 (1995), to appear in Commun. Math. Phys. 
[46] Novikov S.P., Russ. Math. Surveys 37:5, 1 (1982).

[47] Reshetikhin N.Yu., Semenov-Tian-Shansky M.A., Lett. Math. Phys. 19, 133 (1990).

[48] Reshetikhin N.Yu., Turaev V.G., Commun. Math. Phys. 127, 1 (1990).

[49] Sweedler M.E.: Hopf algebras. New York: Benjamin Press 1969.

[50] Wess J., Zumino B., Phys. Lett. B 37, 95 (1971).

[51] Witten E., Commun. Math. Phys. 92, 455 (1984). 